\title{
Nouveaux paresseux terrestres (Mammalia, Xenarthra, Mylodontidae) du Néogène de l'Altiplano bolivien
}

\author{
Pierre-Antoine SAINT-ANDRÉ \\ Le Montaumarchand, F-87190 Droux (France)
}

François PUJOS

Dpto de Geología y Paleontología, Instituto Argentino de Nivología, Glaciología y Ciencias Ambientales (IANIGLA), CCT-CONICET-Mendoza, Avda. Ruiz Leal s/n, Parque Gral. San Martín, 5500 Mendoza (Argentine)

fpujos@lab.cricyt.edu.ar

Cástor CARTELLE

PUC Minas, Avenida Dom José Gaspar, 290 Coração Eucarístico, Belo Horizonte, Minas Gerais (Brésil) cartelle@pucminas.br

Gerardo DE IULIIS

Faculty of Community Services and Health Sciences, George Brown College of Applied Arts and Technology, 200 King Street East, Toronto, Ontario, M5A 1J5 (Canada) gerry.deiuliis@utoronto.ca

Timothy J. GAUDIN

Department of Biological and Environmental Sciences (Department 2653), University of Tennessee at Chattanooga, 615 McCallie Avenue, Chattanooga, TN 37403-2598 (États-Unis) timothy-gaudin@utc.edu

H. Gregory McDONALD

Park Museum Management Program, National Park Service, 1201 Oakridge Drive, Suite 150, Fort Collins, Colorado 80525 (États-Unis) Greg_McDonald@nps.gov

Bernardino MAMANI QUISPE

Departamento de Paleontología, Museo Nacional de Historia Natural, Calle 26 s/n, Cota Cota, La Paz (Bolivie) bmamaniq@hotmail.com Néogène de l'Altiplano bolivien. Geodiversitas 32 (2): 255-306. 
MOTS CLÉS

Mammalia,

Xenarthra,

Tardigrada,

Mylodontidae,

Néogène,

Huayquérien,

Montehermoséen,

Altiplano bolivien,

anatomie,

genre nouveau,

espèces nouvelles.

\title{
RÉSUMÉ
}

Deux nouveaux Mylodontinae (Xenarthra, Tardigrada, Mylodontoidea) provenant de l'Altiplano bolivien sont décrits. L'un, Pleurolestodon dalenzae n. sp., a été découvert à quelques mètres sous un tuf volcanique servant de repère stratigraphique, la Toba 76, daté à 5,4 Ma; il pourrait être d'âge huayquérien (Miocène supérieur) ou à la limite Huayquérien-Montehermoséen. Simomylodon uccasamamensis n. gen., n. sp., a été découvert dans plusieurs gisements d'une formation comprenant la Toba 76 à sa base et un tuf volcanique daté à 2,8 Ma à son sommet; il est d'âge Montehermoséen-Chapadmalaléen (Pliocène inférieur et moyen). L'étude de ces taxons montre que ce sont des Mylodontidae plus proches de Glossotherium, Glossotheridium, Kiyumylodon et Paramylodon que de Lestodon ou Thinobadistes.

\begin{abstract}
New ground sloths (Mammalia, Xenarthra, Mylodontidae) from the Neogene of the Bolivian Altiplano.

Two new Mylodontinae (Xenarthra, Tardigrada, Mylodontoidea) from the Bolivian Altiplano are described. One of them, Pleurolestodon dalenzae n. sp.,

was discovered a few meters below a volcanic tuff, Toba 76, that may be used as a reference stratigraphic level and is dated at $5.4 \mathrm{Ma}$; it could be Huayquerian (late Miocene) or at the Huayquerian-Montehermosan boundary in age. The other taxon, Simomylodon uccasamamensis n. gen., n. sp., was recovered from many localities of a horizon bounded below by Toba 76 and above by another volcanic level, the $2.8 \mathrm{Ma}$ Ayo Ayo tuff; its age is MontehermosanChapadmalalan (early and middle Pliocene). Analysis of these taxa indicates that they are Mylodontidae more closely related to Glossotherium, Glossotheridium, Kiyumylodon and Paramylodon than to Lestodon or Thinobadistes.
\end{abstract}

KEY WORDS

Mammalia,

Xenarthra,

Tardigrada,

Mylodontidae,

Neogene,

Huayquerian,

Montehermosan,

Bolivian Altiplano, anatomy, new genus, new species.

\section{PRÉAMBULE}

Durant la période 1989-1990, P.-A. Saint-André a visité à plusieurs reprises un grand nombre de gisements fossiliferres du nord et du centre de l'Altiplano bolivien. Les nombreuses et importantes découvertes qu'il réalise ont grandement facilité ses recherches, qui ont abouti en 1994 à la présentation d'une thèse de doctorat. À partir du début des années 2000, l'un de nous (F. Pujos), réalisant une thèse de doctorat, puis un post-doctorat sur les paresseux géants terrestres, suit la trace de son aîné sur des sites péruviens, brésiliens et boliviens. Dans ces derniers, il confirme la stratigraphie du site d'Ayo Ayo et découvre de nombreux fossiles sur l'Altiplano (en particulier de nombreux restes attribuables au genre Simomylodon n. gen.) qui l'amènent tout naturellement à entrer en contact avec P.-A. Saint-André. Celui-ci lui fait part d'un manuscrit en attente, fondamental pour notre connaissance des paresseux géants, tant par l'importance des fossiles décrits (deux nouvelles formes) que par leur intérêt stratigraphique et phylogénétique. Un groupe de chercheurs spécialistes de Xénarthres est constitué et, en parfaite intelligence avec P.-A. Saint-André, propose une révision du manuscrit et encourage sa parution pour que les importants résultats de cette thèse inédite soient mis à la disposition de la communauté scientifique. Tout en conservant la plupart des idées originelles défendues par P.-A. Saint-André, ce texte bénéficie des connaissances les plus récentes sur le sujet. Nous le présentons aujourd'hui. 


\section{INTRODUCTION}

\section{PRÉSENTATION CHRONOSTRATIGRAPHIQUE}

Le premier et le plus ancien de ces deux taxons, Pleurolestodon dalenzae n. sp., est connu par le crâne holotype. Il a été trouvé près du village de Choquecota (Fig. 1) à 15 mètres en dessous de la Toba 76, tuf volcanique repère, daté à $5,4 \mathrm{Ma}$ (Marshall et al. 1992; Saint-André 1994). Ce fossile proviendrait soit des niveaux supérieurs de la Formation Rosa Pata (Conglomérat Pomata, Miocène supérieur), soit des niveaux inférieurs de la Formation Umala (Marshall \& Sempéré 1991); ces données permettent de situer $P$. dalenzae n. sp. à la limite du Huayquérien (Miocène supérieur) et du Montehermoséen (Pliocène inférieur et moyen).

Le second Mylodontidae Gill, 1872, Simomylodon uccasamamensis n. gen., n. sp., avait déjà été signalé dans la Formation Umala, dans le gisement d'Ayo Ayo-Viscachani (Hoffstetter et al. 1971) ainsi que dans la Formation Remedios (Lavenu 1984) (Fig. 1). Le Mylodontidae du gisement pliocène de Pomata (Hoffstetter et al. 1972) est référé à ce dernier taxon. Ce gisement correspond à la Formation Mauri qui repose sur la Formation Rosa Pata d'âge huayquérien, et plus particulièrement sur le faciès terminal de cette dernière, le "Conglomérat Pomata (Marshall \& Sempéré 1991). Cette "Formation Mauri» est probablement différente de la formation homonyme de l'Oligocène et du Miocène de la Cordillère Occidentale et de l'Ouest de l'Altiplano et représente un équivalent stratigraphique de la Formation Umala. La base de cette dernière peut être datée grâce à la présence de la Toba 76 et son sommet grâce au "tuf volcanique Ayo Ayo ", daté à 2,8 Ma.

\section{GÉNÉRALITÉS SUR LES MYLODONTIDAE}

Les Mylodontidae constituent la famille nominative de la superfamille Mylodontoidea Gill, 1872 (Scillato-Yané 1977; Engelmann 1985; Hirschfeld 1985 ; Esteban 1996). Ces derniers sont caractérisés notamment par la morphologie particulière de la mandibule qui présente le bord inférieur de la branche horizontale relativement droit, le processus

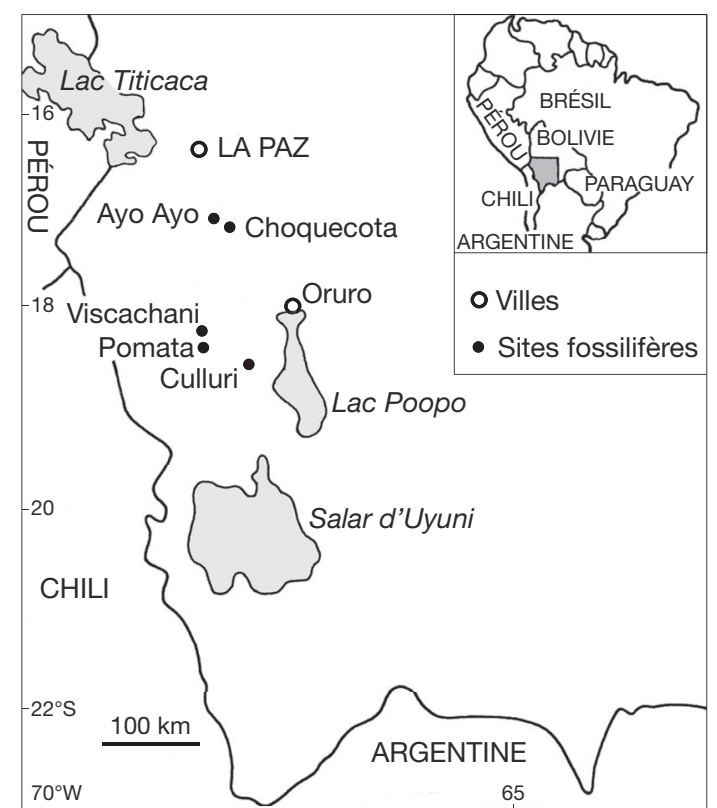

FIG. 1. - Gisements fossilifères du Néogène de l'Altiplano bolivien $(\bullet)$ ayant livré les restes de Pleurolestodon dalenzae n. sp. et Simomylodon uccasamamensis n. gen., n. sp.

angulaire proéminent et le processus coronoïde de la branche ascendante proportionnellement bas (Engelmann 1985; Saint-André 1994) et les molariformes postérieures bilobées, caractère qu'ils partagent avec le "paresseux-glyptodonte» déséadien aux affinités incertaines, Pseudoglyptodon Engelmann, 1987 (Engelmann 1987 ; McKenna et al. 2006; Pujos \& De Iuliis 2007).

Les taxons du Déséadien d'Argentine Orophodon hapaloides Ameghino, 1895 et Octodontotherium grande Ameghino, 1895, précédemment classés au sein des Paragravigrades notamment par Hoffstetter $(1956$; 1958 ; 1982) et Paula Couto (1979), sont désormais attribués soit aux Mylodontidae (Gaudin 2004) soit aux Orophodontidae Ameghino, 1895 (Pujos \& De Iuliis 2007). La révision de l'ensemble des restes de paresseux «Mylodontoides " provenant du Déséadien est essentielle. Le clade Orophodontidae est-il monophylétique au sein des Mylodontoidea ou fait-il partie à part entière du clade Mylodontidae (Pujos 2007; Pujos \& De Iuliis 2007; Pujos et al. 2008)? 
Pseudoprepotherium Hoffstetter, 1961, du Santacruzien tardif et du Chasicoïen de Colombie et du Venezuela, est inclus dans la famille des Mylodontidae par Hirschfeld (1985) mais en est exclu par Engelmann (1985) qui en fait le taxon frère d'Orophodon Ameghino, 1895. Gaudin (2004) le considère comme le Mylodontinae Gill, 1872 le plus primitif.

Carlini et al. (2006) ont présenté deux nouveaux genres vénézuéliens de Mylodontidae du Miocène moyen-Pliocène: Mirandabradys Carlini, Scillato-Yané \& Sánchez (Mylodontoidea aux affinités incertaines, représenté par trois espèces) et Bolivartherium Carlini, Scillato-Yané \& Sánchez, 2006 (Lestodontinae Ameghino, 1889 représenté par deux espèces fortement semblables). Les restes abondants, mais particulièrement mal conservés, de ces deux genres ne permettent pas, à ce jour, de préciser leurs positions phylogénétiques.

La famille Mylodontidae regroupe différentes sous-familles selon les auteurs considérés. Dans un travail récent (Rinderknecht et al. 2007) les deux sous-familles Mylodontinae et Scelidotheriinae Ameghino, 1889 composent le clade Mylodontidae. Carlini et al. (2006) considèrent également comme valide la sous-famille Lestodontinae. Rinderknecht et al. (2007) confirment l'opinion originale de Scillato-Yané (1977) à savoir que le genre Octomylodon Ameghino, 1904 constitue un clade particulier de Mylodontidae (5/4 dents molariformes, toutes bilobées) qui correspond à la sousfamille Octomylodontinae Scillato-Yané, 1977. Cependant, les caractéristiques particulières d'Octomylodon pourraient indiquer que ce genre est le groupe frère des Mylodontidae, comme le suggère Gaudin (2004).

Negri \& Ferigolo (2004) créent une cinquième sous-famille, Urumacotheriinae Negri \& Ferigolo, 2004, uniquement composée du genre Urumacotherium Bocquentin-Villanueva, 1983 du Miocène supérieur du Venezuela. Ce nouveau genre correspond également à Acremylodon Mones, 1986 et Stenodon Frailey, 1986 (Frailey 1986; Mones 1986), qui en sont des synonymes plus récents. Urumacotherium comprend 2 espèces: $U$. garciai Bocquentin-Villanueva, 1984 et $U$. campbelli (Frailey, 1986). La seconde espèce est représentée par un crâne complet mais dont les dents n'ont pas été conservées (LACM 117502). Par contre, la création d'une quatrième sous-famille de Mylodontidae basée sur la seule présence d'un caractère (forte compression antéropostérieure des dents supérieures) nous paraît peu convainquante. Pour cette raison, il nous semble plus prudent d'attendre une analyse phylogénétique rigoureuse et détaillée des Mylodontidae, avant de considérer le genre Urumacotherium comme constituant une sousfamille à part entière de Mylodontidae.

Considérant que la subdivision de la famille Mylodontidae diverge radicalement en fonction des auteurs considérés, nous utiliserons les clades définis dans le cadre de l'analyse phylogénétique des tardigrades la plus complète (Gaudin 2004), dans laquelle deux sous-familles, Mylodontinae et Scelidotheriinae, sont monophylétiques. Dans cette analyse, les paresseux «Lestodondines» (i.e. Thinobadistes Hay, 1919 et Lestodon Gervais, 1855) font partie du clade Mylodontinae, constituant la tribu Lestodontini Ameghino, 1889, ainsi défini préalablement par Webb (1989). Finalement, le genre atypique provenant du chasico-huayquérien argentin Octomylodon est considéré comme le groupe frère des autres paresseux mylodontoides (Gaudin 2004) mais sa morphologie très particulière (mélange unique de caractères Mylodontides et Mégathérides) rend difficile l'établissement de relations avec les deux sous-familles Mylodontinae et Scelidotheriinae. Octomylodon est un Tardigrade Mylodontoïde particulier (représenté par un seul crâne et une mandibule) dont la position phylogénétique est incertaine mais qui pourrait correspondre au groupe frère des Mylodontidae (Gaudin 2004).

À la sous-famille Scelidotheriinae, nous associons les genres:

- Nematherium Ameghino, 1887 du Santacruzien (Miocène moyen);

- Scelidotherium Owen, 1839 du Lujanien (Pléistocène récent);

- Scelidodon Ameghino, 1881 du Lujanien;

- Catonyx Ameghino, 1891 également du Lujanien;

- Proscelidodon Bordas, 1935 du Montehermoséen et Chapadmalalien (Miocène supérieur-Pliocène 
inférieur) argentin et bolivien (Anaya \& MacFadden 1995). Pujos (2000) estime que le genre Scelidodon est valide alors que McDonald \& Perea (2002) le considèrent comme synonyme de Catonyx.

À la sous-famille Mylodontinae se rapportent les genres:

- Pleurolestodon Rovereto, 1914 du Huayquérien (Miocène supérieur) d'Argentine;

- Ranculcus Ameghino, 1891 du Huayquérien;

- Sphenotherus Ameghino, 1891 également du Huayquérien (Rovereto [1914] et Cione et al. [2000] évoquent ces 3 genres argentins);

- Thinobadistes de l'Hemphillien (Miocène supérieur) du Texas et de Floride;

- Glossotheridium Kraglievich, 1934 du Montehermoséen argentin (Miocène supérieur et Pliocène inférieur), également signalé dans le Montehermoséen et le Chapadmalalien de Bolivie (du Miocène supérieur au Pliocène moyen [Anaya \& MacFadden 1995]);

- Glossotherium Owen, 1839 et Paramylodon Brown, 1903 du Marplatien, de l'Ensénadien, du Lujanien (Pléistocène) d'Amérique du Sud et du Pléistocène nord-américain;

- Mylodon Owen, 1839 de l'Ensénadien et du Lujanien (Pléistocène) d'Argentine, d'Uruguay et du Chili;

- Urumacotherium du Huayquérien des Formations Urumaco (Venezuela) et Solimóes (frontière entre le Pérou et le Brésil);

- Kiyumylodon Rinderknecht, Perea \& McDonald 2007 du Huayquérien d'Uruguay;

- Oreomylodon Hoffstetter, 1949 du Lujanien (Quaternaire récent) d'Équateur;

- Lestodon de l'Ensénadien et du Lujanien d'Argentine, d'Uruguay, du Brésil et de Bolivie;

- Mylodonopsis Cartelle, 1991 du Lujanien du Brésil;

- Promylodon Ameghino, 1883 du Miocène argentin;

- Bolivartherium Carlini, Scillato-Yané \& Sánchez, 2006 du Miocène supérieur et du Pliocène du Venezuela.

Comme mentionné précédemment, ces deux derniers taxons sont regroupés par Gaudin (2004) au sein des Mylodontinae-Lestodontini.

\section{ABRÉVIATIONS}

Institutions et collections

GB ex «Geogol» (= Servicio Geológico de Bolivia) et actuel Servicio Geológico Técnico Minero, La Paz, Bolivie;

IFEA Institut français d'Études andines, Lima, Pérou;

MACN-Pv MACN, División de Paleontología de Vertebrados, Museo Argentino de Ciencias Naturales Bernardino Rivadavia, Buenos Aires, Argentine;

MCL Museu de Ciencias Naturais da Pontifícia Universidade Católica de Minas Gerais, Belo Horizonte, Brésil;

MLP Museo de La Plata, La Plata, Argentine; MNHN Muséum national d'Histoire naturelle, Paris, France;

AYO collection Ayo Ayo (Bolivie);

PAM collection du Pampéen (Argentine);

POM collection Pomata (Bolivie);

VIZ collection Viscachani (Bolivie);

MNHN-BOL Museo Nacional de Historia Natural, La Paz, Bolivie;

UCMP University of California Museum of Paleontology, Berkeley, États-Unis.

\section{Ostéologie \\ C- dent caniniforme supérieure; \\ C. dent caniniforme inférieure; \\ M dent molariforme supérieure; \\ m dent molariforme inférieure.}

\section{SYSTÉMATIQUE}

Super-famille MylodonTOIDEA Gill, 1872

Diagnose. - Molariformes postérieures fortement bilobées; bord inférieur de la branche horizontale relativement droit; processus angulaire proéminent; processus coronoïde de la branche ascendante relativement bas.

\section{Famille MyLOdOnTIDAE Gill, 1872}

Diagnose. - Dents prismatiques, de section transversale arrondie, ovale, elliptique ou triangulaire, pouvant présenter un ou des sillons le long du fût des dents antérieures; $\mathrm{M} 4 / \mathrm{m} 3$ en forme de « 8 "; la couche de cément périphérique est mince, la couche d'ostéodentine est épaisse; perte de la crête du bord antéromédial du processus coronoïde de la mandibule; mandibule avec le col du condyle articulaire court; condyle mandibulaire en 
TABLEAU 1. - Principales dimensions (en $\mathrm{mm}$ ) du crâne holotype de Pleurolestodon dalenzae n. sp. (MNHN-BOL V 3348).

\begin{tabular}{|c|c|}
\hline Longueur de la C- & 4,6 \\
\hline Largeur de la C- & 0,8 \\
\hline $\begin{array}{l}\text { Hauteur de la C- du bord alvéolaire externe } \\
\text { à l'apex }\end{array}$ & 27 \\
\hline Longueur de la M1 & \\
\hline argeur de la M1 & \\
\hline Hauteur de la M1 & \\
\hline Longueur maximale de la M2 & \\
\hline Longueur de la M2 face linguale & \\
\hline Largeur de la M2 & \\
\hline Hauteur de la M2 & \\
\hline Longueur maximale de la M3 & \\
\hline le la M3 face & \\
\hline Largeu & \\
\hline Hautel & \\
\hline e la M4 & \\
\hline ale du lobe antérieur de & \\
\hline Larg & \\
\hline${ }_{4}$ inimale au niveau de l'isthme central & \\
\hline u lobe antérieur de la M4 & \\
\hline laute & \\
\hline série & 10 \\
\hline lais entr & \\
\hline M1 & \\
\hline 12 & \\
\hline 13 & \\
\hline ais en & \\
\hline $\begin{array}{l}\text { ntérieur du maxillaire } \\
\text { chancrure postpalatine }\end{array}$ & 11 \\
\hline $\begin{array}{l}\text { laximale du museau sur le renflement } \\
\text { re }\end{array}$ & 8 \\
\hline $\begin{array}{l}\text { Distance entre les bords alvéolaires externes } \\
\text { des } \mathrm{C}^{-}\end{array}$ & 80 \\
\hline $\begin{array}{l}\text { Distance minimale entre M4 et le sommet de } \\
\text { l'échancrure postpalaltine }\end{array}$ & 23 \\
\hline $\begin{array}{l}\text { Largeur minimale du museau en avant des } \\
\text { apophyses zygomatiques }\end{array}$ & 64 \\
\hline $\begin{array}{l}\text { Diamètre minimal en arrière des processus } \\
\text { postorbitaires des frontaux }\end{array}$ & 54 \\
\hline 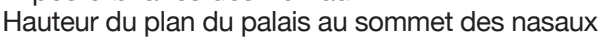 & \\
\hline Indic & \\
\hline
\end{tabular}

position basse (situé au même niveau ou juste au dessus de la série dentaire); bords latéraux du "bec» de la mandibule parallèles; processus zygomatique du squamosal court; membre antérieur relativement court et puissant dont les os portent de fortes crêtes pour l'insertion des muscles; main penta- à tétradactyle (disparition du doigt I) dont les phalanges unguéales portent des griffes plus fortes que celles des pieds et qui ont tendance à s'aplatir dorsoventralement; fémur relativement élargi; la fossette pour le ligament rond est réduite à une échancrure sur le bord postérieur de la surface articulaire de la tête; le troisième trochanter, assez bas, disparait sur les formes les plus évoluées; tibia et fibula sont courts et généralement libres (rarement soudés à leur seule extrémité distale); pied tétra- ou tridactyle (Pascual 1966) avec disparition des doigts I ou I-II.

\section{Sous-famille MYLOdonTINAE Gill, 1872}

Diagnose. - Denture hétérodonte; premières dents antérieures supérieure et inférieure caniniformes, ayant tendance à être positionnées vers l'extérieur et pouvant s'isoler des autres dents par un diastème; C- et $\mathrm{C}_{\text {_ }}$ parfois absente (e.g., Mylodon); M1-M3/m1-m2 de forme arrondie avec une face linguale aplatie et marquée d'un léger sillon longitudinal, à grossièrement triangulaire, rarement rectangulaire; les deux lobes de la M4 et de la m3 séparés par un isthme plus ou moins allongé; les séries dentaires ont tendance à s'écarter d'arrière en avant; M2-M3 de section triangulaire comprimée antéropostérieurement; lobe postérieur de la M4 allongé et bien séparé du lobe antérieur; crâne de forme générale cylindrique; le museau a tendance à s'élargir vers l'avant ; prémaxillaires généralement courts et larges; bord latéral des maxillaires ne se prolongeant pas en avant de l'alvéole de la C-; symphyse mandibulaire ayant tendance à s'élargir jusqu'à atteindre une forme de pelle chez les genres les plus évolués; sacrum allongé; pelvis très grand aux ilions très étalés; humérus sans pont entépicondylien (absence $\mathrm{du}$ foramen entépicondylien); main pentadactyle avec griffes aux doigts I à III (I à IV selon Pascual [1966]), la griffe du doigt III étant la plus forte; fémur aplati mais peu élargi (surtout distalement); facette cuboïdienne de l'astragale plane à légèrement convexe; calcanéum massif; pied tétradactyle (absence d'un doigt I fonctionnel); les doigts II-III portent des griffes moyennement développées; les doigts IV-V sans phalange unguéale; métatarsiens IV-V robustes, sans expansion postéroexterne (d'après Saint-André [1994]).

\section{Genre Pleurolestodon Rovereto, 1914}

ESPÈCE INCLUSES. $-P$. acutidens $(=P$. macrodon et $P$. avitus); P. dalenzae n. sp.

Diagnose. - Mylodontinae de petite taille (voir Tableau 1), plus petit que Glossotheridium chapadmalense (Kraglievich, 1925) mais plus grand que Simomylodon uccasamamensis n. gen., n. sp.; séries dentaires fortement divergentes (cependant moins que chez le genre Quaternaire Glossotherium), dépourvues de diastème; le rapport de la distance entre les faces internes des C d'une part, et celle entre les faces internes des M4 d'autre 
part, varie entre 1,61 ( $P$. acutidens) et 2,21 ( $P$. dalenzae n. sp.); la base antérieure de l'arcade zygomatique se situe sur les parois latérales du maxillaire à un niveau situé entre le milieu de la M2 et l'extrémité postérieure de la M3 (comme chez Pseudoprepotherium), la section de la M2 est allongée antéropostérieurement, en forme de triangle rectangle d'angle droit antérolingual, avec sillon lingual large et profond et face postérolabiale très longue; l'extrémité postérieure du palais est nettement en arrière du plan transversal passant par les processus post-orbitaires; les nasaux s'immisçant en pointe dans le bord antérieur des frontaux; présence d'un petit élément internasal pair se projetant vers l'avant à l'extrémité médio-antérieure des nasaux.

\section{REMARQUE}

Le genre Pleurolestodon a été défini par Rovereto (1914) sur la base de matériel provenant du Huayquérien (Miocène supérieur) de la Province de Catamarca (Argentine). Initialement, Rovereto (1914) définit trois espèces constituant le genre Pleurolestodon: P. avitus Rovereto, 1914 (spécimens types: MACN-Pv 2785 et 2952), P. acutidens Rovereto, 1914 (spécimen type: MACN-Pv 2953) et $P$. macrodon Rovereto, 1914 (spécimen type: MACN-Pv 2784). Kraglievich (1921) effectuant une révision du matériel décrit préalablement par Rovereto et déposé au MACN de Buenos Aires, estime que $P$. macrodon est synonyme de P. acutidens. En cela nous suivons l'opinion présentée par Kraglievich (1921). De plus comme le signalait Rovereto (1914), $P$. avitus présente de très nombreuses affinités avec P. macrodon et les seules différences «notables» présentées par Rovereto (1914), principalement dentaires, ne nous semblent pas suffisantes pour définir deux espèces de Pleurolestodon au sein du matériel provenant de Catamarca. Pour cette raison, nous considérions jusqu’à la réalisation du présent manuscrit, Pleurolestodon comme monospécifique. Pleurolestodon acutidens est l'unique espèce valide du Huayquérien de Catamarca. L'holotype de $P$. acutidens est le spécimen MACN-Pv 2953 alors que les spécimens MACN-Pv 2784, 2785 et 2952 sont désormais considérés comme paratypes.

Les deux espèces qui composent ainsi le genre Pleurolestodon proviennent du Miocène supérieur (Huayquérien-Montehermoséen) d'Argentine (Province de Catamarca $-P$. acutidens) et de Bolivie (Département d'Oruro - P. dalenzae n. sp.).
Une révision récente par A. Kramarz du matériel attribué à Pleurolestodon et déposé au MACN, a permis de noter l'absence du spécimen type de P. acutidens (MACN-Pv 2953, A. Kramarz pers. comm. 2008). Cependant la description détaillée et les excellentes illustrations présentes dans la publication de Rovereto (1914) nous ont permis d'utiliser le spécimen type de $P$. acutidens.

\section{Pleurolestodon dalenzae n. $\mathrm{sp}$.} (Figs 2-4; Tableau 1)

«Glossotheriscum dalenzae» - Saint-André 1994: 174183, fig. 18, pl. 13 (unpublished).

ÉTYMOLOGIE. — En l'honneur d'Alejandra Dalenz Farjat, paléontologue bolivienne, qui a participé activement au dégagement sur le terrain du spécimen type.

Holotype et Seul spécimen CONNU. - MNHN-BOL V 3348 (Figs 2-4;Tableau 1), crâne partiel où manquent les régions occipitale, basi-crânienne et auditives ainsi que les arcades zygomatiques, la C- gauche et la M4 droite.

LOCAlité TYPE. - Sud-ouest de Choquecota (Département d'Oruro, Province de Carangas, Bolivie), à $15 \mathrm{~m}$ sous la Toba 76, au-dessus de la rive orientale du fleuve Barras (Fig. 1).

NiVEAU TYPE. - Spécimen découvert à une quinzaine de mètres sous le niveau repère qu' est la Toba 76 . Le crâne type est légèrement plus ancien que ce niveau repère daté à 5,4 Ma par Marshall et al. (1992) et Evernden et al. (1977). La présence d'une discontinuité sous la Toba 76 a été notée par Hoffstetter et al. (1972), Lavenu (1986) et Saint-André (1994). À Pomata, par exemple, le niveau volcanique repose en faible discordance sur les terrains du Miocène supérieur. Selon toute vraisemblance, ce fossile est contemporain de cette époque. Cependant, le fait que Lavenu (1986) ait noté, à Vituy de Umala, l'existence de niveaux conglomératiques et sableux épais de 8 à $10 \mathrm{~m}$ à la base de la séquence pliocène et sur lesquels la Toba 76 repose en concordance, ne permet pas d'être catégorique quant à l'attribution du stratum typicum de Pleurolestodon dalenzae n. sp. à la Formation Rosa Pata (Miocène supérieur) ou à la Formation Umala (Pliocène inférieur et moyen). En effet dans la région de Choquecota aucune discordance n'apparaît dans les niveaux détritiques sous-jacents à la Toba 76, discordance qui aurait pu suggérer le passage Miocène-Pliocène. Il est donc difficile d'estimer l'âge du niveau sableux d'où provient le crâne de Pleurolestodon dalenzae n. sp. Appar- 
tient-il au sommet de la séquence Miocène supérieur ou à la base de la séquence Pliocène?

Diagnose. - Crâne moins massif que chez $P$. acutidens, forte constriction transverse surtout observable au niveau des frontaux; $C$ - très allongée; la section de la C- est semi-circulaire (légèrement triangulaire chez P. acutidens); plus forte divergence des séries dentaires (rapport de la distance entre les faces internes des C- à celle entre les faces internes des M4 est de 2,21 au lieu de 1,61 chez $P$. acutidens); face linguale de la M1 convexe lingualement (concave chez $P$. acutidens) ; léger sillon sur la face médiale de la M1; M2 faiblement compressée antéropostérieurement en comparaison avec $P$. acutidens; M2 présentant un très fort sillon lingual; lobe postérieur de la M4 allongé et étroit.

Description COMPARATIVE (FIgs 2-4; TABleaU 1) Dents supérieures (Figs 2; 3; Tableau 1)

Les séries dentaires sont continues comme chez Pseudoprepotherium confusum Hirschfeld, 1985, Pleurolestodon acutidens, Simomylodon uccasamamensis n. gen., n. sp., Glossotheridium chapadmalense, Paramylodon harlani (Owen, 1839), Mylodon darwini Owen, 1839, Urumacotherium campbelli et Mylodonopsis ibseni Cartelle, 1991, alors que chez Glossotherium robustum (Owen, 1842) et le Lestodontini Lestodon armatus Gervais, 1855, un diastème existe entre C- et M1.

Comme la distance entre les faces linguales des C- est 2,21 fois plus grande que celle séparant les faces linguales des M4 (Fig. 3), les séries dentaires divergent nettement plus que chez Urumacotherium campbelli (à cause du fort diamètre transverse de l'alvéole de la première dent supérieure [Frailey 1986]), Paramylodon harlani, Pleurolestodon acutidens (Kraglievich 1921), Mylodonopsis ibseni (Cartelle 1991) et Glossotheridium chapadmalense (Kraglievich 1925) où les rapports sont respectivement de $1,33,1,56,1,61,1,66$ et 1,97. Cette divergence est comparable au rapport de 2,22 observable chez le spécimen MNHN-BOL V 3321 de Simomylodon uccasamamensis n. gen., n. sp. Chez Glossotherium robustum MNHN PAM 141 et G. wegneri (Spillmann, 1931) ce rapport est de 2,45 et 2,05 respectivement.

Les caractères dentaires ne sont pas applicables à Mylodon darwini, qui a perdu la première dent supérieure, provoquant ainsi une notable modification des dents suivantes.
Les alvéoles des dents d'Urumacotherium campbelli présentent une très forte compression antéropostérieure, absente chez tous les autres représentants du clade Mylodontinae.

La première dent supérieure est clairement caniniforme (comme définie par Pujos \& De Iuliis [2007]), relativement peu développée par rapport à Glossotherium mais surtout Lestodon et très légèrement courbée vers l'arrière (Fig. 2). Elle ne présente pas de courbure médiale comme chez $G$. wegneri. La section de la C- est semi-circulaire chez Pleurolestodon dalenzae n. sp. (Fig. 3), légèrement triangulaire chez P. acutidens (FMNH P14495), Simomylodon n. gen. (MNHN-BOL V 3321) ou G. wegneri, voire elliptique chez $G$. robustum et Mylodonopsis ibseni (MCL-4355). La C- n'est pas conservée chez le seul crâne connu de Pseudoprepotherium (UCMP 39957; Hirschfeld 1985). La face antéromédiale de la dent est plane. Le grand axe de la section est dirigé antéropostérieurement et latéralement vers l'avant. La surface d'usure forme un biseau dirigé vers l'arrière et sensiblement médialement (Fig. 3).

La partie extra-alvéolaire du fût de la M1 est droite, moins courbée vers l'arrière que chez le paratype MNHN-BOL V 3321 de Simomylodon uccasamamensis n. gen., n. sp. ou chez Glossotherium wegneri. Sa section est grossièrement elliptique et son grand axe est parallèle à celui de la $\mathrm{C}$ (Fig. 3). Le rayon de courbure de l'extrémité antérieure de la section est plus petit que celui de l'extrémité postérieure. La face médiale présente un léger sillon longitudinal plus proche du bord antérieur que du bord postérieur, contrairement à ce que l'on observe chez Pleurolestodon acutidens (FMNH P14495; Rovereto 1914) et Paramylodon harlani (Kraglievich 1921), sillon absent chez Glossotheridium chapadmalensis (MLP M-685) et semble-t-il chez Pseudoprepotherium confusum (UCMP 39957). La face linguale de la M1 est convexe lingualement chez $P$. dalenzae n. sp. et concave sur les spécimens MACN Pv 2953 (Rovereto 1914) et FMNH P14495 de P. acutidens. La surface d'usure de la M1, divisée en deux biseaux, devait avoir une fonction intermédiaire entre une caniniforme et une molariforme comme chez P. acutidens. Le biseau antérieur, légèrement convexe, est moins étendu que celui de la surface postérieure (Figs 2; 3). Cette dernière, concave suivant sa hau- 

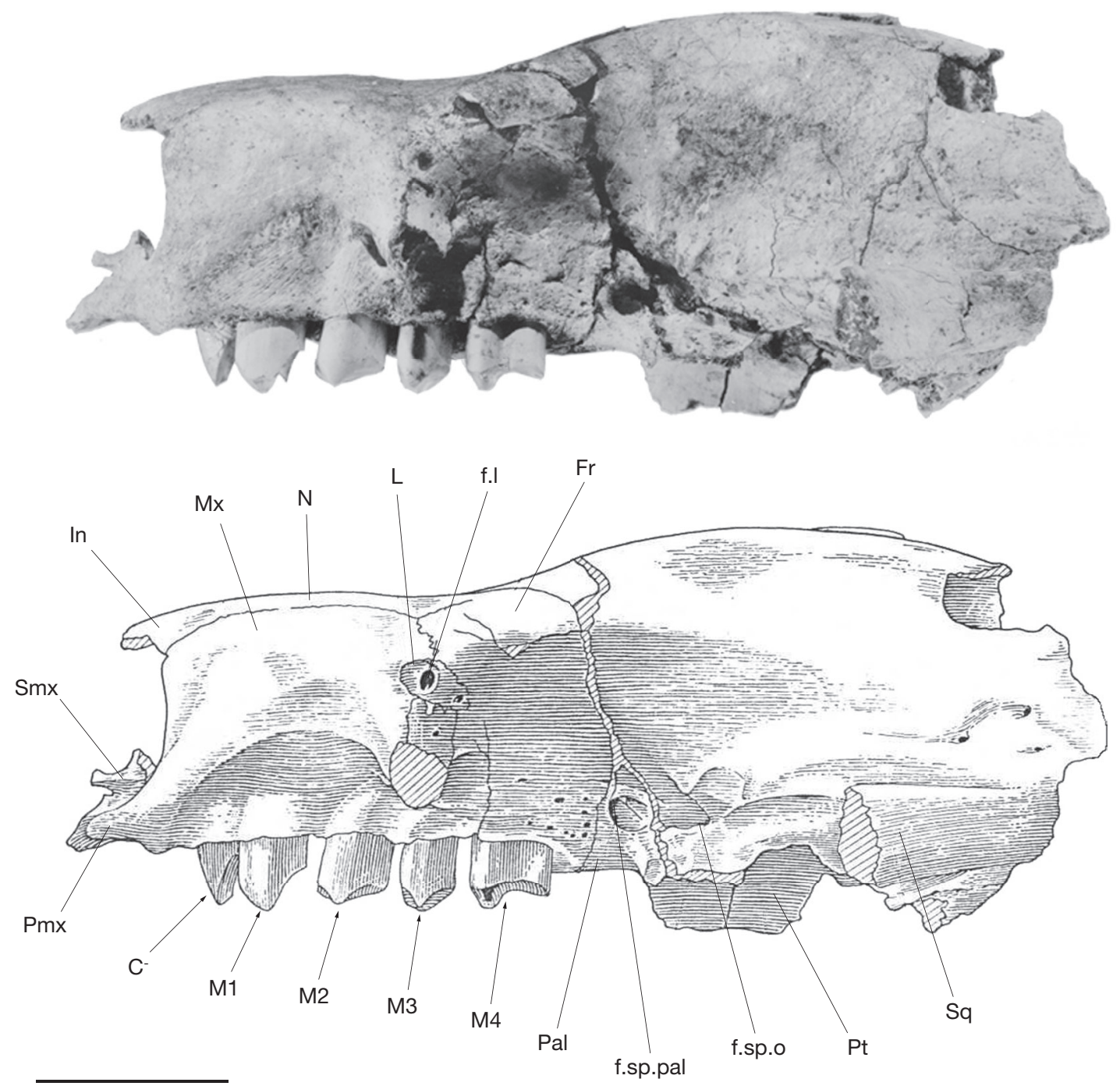

FIG. 2. - Crâne holotype de Pleurolestodon dalenzae n. sp. (MNHN-BOL V 3348) en vue latérale (antérieure vers la gauche). Abréviations: C-, dent caniniforme supérieure; f.I, foramen lacrymal; Fr, frontal; f.sp.o, fissure sphénorbitaire; f.sp.pal, foramen sphénopalatin; In, internasal; L, lacrymal; M1-M4, dents molariformes supérieures; Mx, maxillaire; N, nasal; Pal, palatin; Pmx, prémaxillaire; Pt, ptérygoïde; Smx, septomaxillaire; Sq, squamosal. Échelle: $5 \mathrm{~cm}$.

teur, remonte plus haut sur le bord postérieur du fût. La vasodentine centrale forme une dépression centrale étroite, presque entièrement localisée sur la partie postérieure de la surface d'usure. L’arête séparant les deux biseaux, perpendiculaire au grand axe de la dent, est creusée en son centre par l'extrémité antérieure de la dépression de vasodentine qui n'entame qu'à ce niveau le biseau antérieur. La pointe labiale d'ostéodentine est plus haute que la pointe linguale.

Alors qu'elle est ovale et légèrement aplatie lingualement chez Pseudoprepotherium, la M2 de P. dalenzae n. sp. possède une forme générale en triangle rectangle à angle droit en position antérolinguale, caractère également observé chez P. acutidens, Glossotheridium robustum, Simomylodon uccasamamensis 
n. gen., n. sp., Paramylodon harlani et Glossotherium robustum (Fig. 3) mais absent chez Mylodonopsis ibseni (MCL-4355) et Pseudoprepotherium confusum (UCMP 39957). La M2 de P. dalenzae n. sp. est nettement plus compressée antéropostérieurement que chez les spécimens connus de $P$. acutidens. Par contre la face linguale y montre un sillon beaucoup plus large et profond que chez ces taxons et notamment Glossotheridium (MLP M-685) et Paramylodon harlani (Stock 1925). La longueur de la face postérolabiale, marquée en son centre d'un sillon longitudinal comme chez Glossotherium, Paramylodon et Simomylodon n. gen. (P. acutidens et Mylodonopsis, en regard de son alvéole dentaire, en sont dépourvus), est proportionnellement plus grande que chez P. acutidens, Mylodon, Glossotheridium, Simomylodon n. gen. et Glossotherium (ce caractère ne peut s'appliquer à Pseudoprepotherium confusum chez qui la M2 est grossièrement ronde). La surface d'usure est séparée en deux parties par une zone transversale convexe dont les points les plus hauts se situent vers l'extrémité antérieure de la face postérolabiale et près du sillon lingual. La vasodentine centrale forme une dépression.

La M3 ressemble beaucoup à celles de $P$. acutidens, Glossotheridium, Glossotherium robustum, Mylodon darwini, et Simomylodon uccasamamensis n. gen., n. sp. À l'instar des trois premiers taxons, elle est très légèrement plus comprimée antéropostérieurement que la M2 (Fig. 3) alors que chez Simomylodon n. gen., Glossotheridium, Paramylodon et Pseudoprepotherium et Mylodonopsis, M2 et M3 sont presque identiques. Chez Pleurolestodon dalenzae n. sp., l'arête antérolabiale arrondie est plus étroite et le sillon de la face postérolabiale est moins marqué que chez Glossotherium robustum (MNHN PAM 141) et Glossotheridium. La face antérieure de la M3 est moins oblique pour ces deux genres ainsi que pour Simomylodon n. gen. (MNHN-BOL V 3321).

La M4 est bilobée (Fig. 3). Le grand axe du lobe antérieur est orienté antérolabialement à postérolingualement. Le lobe postérieur est plus long et mieux séparé que chez Glossotherium, Glossotheridium, Paramylodon et surtout Mylodonopsis. La dent est semblable à celle de Pseudoprepotherium mais également de $P$. acutidens, à propos de laquelle
Kraglievich (1921) observe que les deux lobes sont mieux définis que chez Glossotherium robustum et plus encore que chez Mylodon garmani Allen, 1913 et Paramylodon nebrascensis Brown, 1903 (nous récusons la validité de ces deux derniers taxons qui doivent être définitivement considérés comme des synonymes de Paramylodon harlani). Le lobe postérieur de la M4 est plus allongé que chez P. acutidens et plus étroit que chez tous les autres Mylodontinae et particulièrement Mylodonopsis.

\section{Crâne (Figs 2-4; Tableau 1)}

Les crânes des deux espèces de Pleurolestodon ( $P$. dalenzae n. sp. et $P$. acutidens), présentent de fortes similitudes avec celui des représentants du genre Glossotherium que Hoffstetter (1952) place parmi les plus petites formes du clade. La taille du crâne de $P$. dalenzae n. sp. est cependant nettement inférieure à ce dernier (voir Tableau 1). En vue dorsale, il présente une allure générale mois massive que chez $P$. acutidens (FMNH P14495), qui se traduit notamment par une plus forte constriction transverse dans la région frontale.

Le museau présente une dilatation importante (Figs 3; 4) qui n'atteint pas le degré observé au Pléistocène chez les différentes espèces du genre Glossotherium.

Chez les deux espèces de Pleurolestodon, comme chez Glossotheridium et Pseudoprepotherium (UCMP 39957; voir Hirschfeld 1985), la surface dorsale des nasaux est plus basse que la surface dorsale de la boîte crânienne contrairement à celle que l'on observe chez Paramylodon harlani où les nasaux sont situés à peine en dessous du toit crânien. La région frontale est légèrement déclive (Fig. 2). La dilatation dorsoventrale du museau est mieux marquée que chez Glossotheridium et la surface dorsale des nasaux est légèrement bombée antéropostérieurement, alors qu'elle est plate chez Glossotheridium et fortement déclive d'arrière en avant chez Pseudoprepotherium. Chez Glossotherium robustum, la surface dorsale du museau est au même niveau que celle de la boîte crânienne, alors que chez G. wegneri, l'extrême dilatation du museau, dans les plans frontaux et sagittaux, amène l'extrémité de celui-ci au-dessus du niveau de la surface dorsale de la boîte crânienne. 

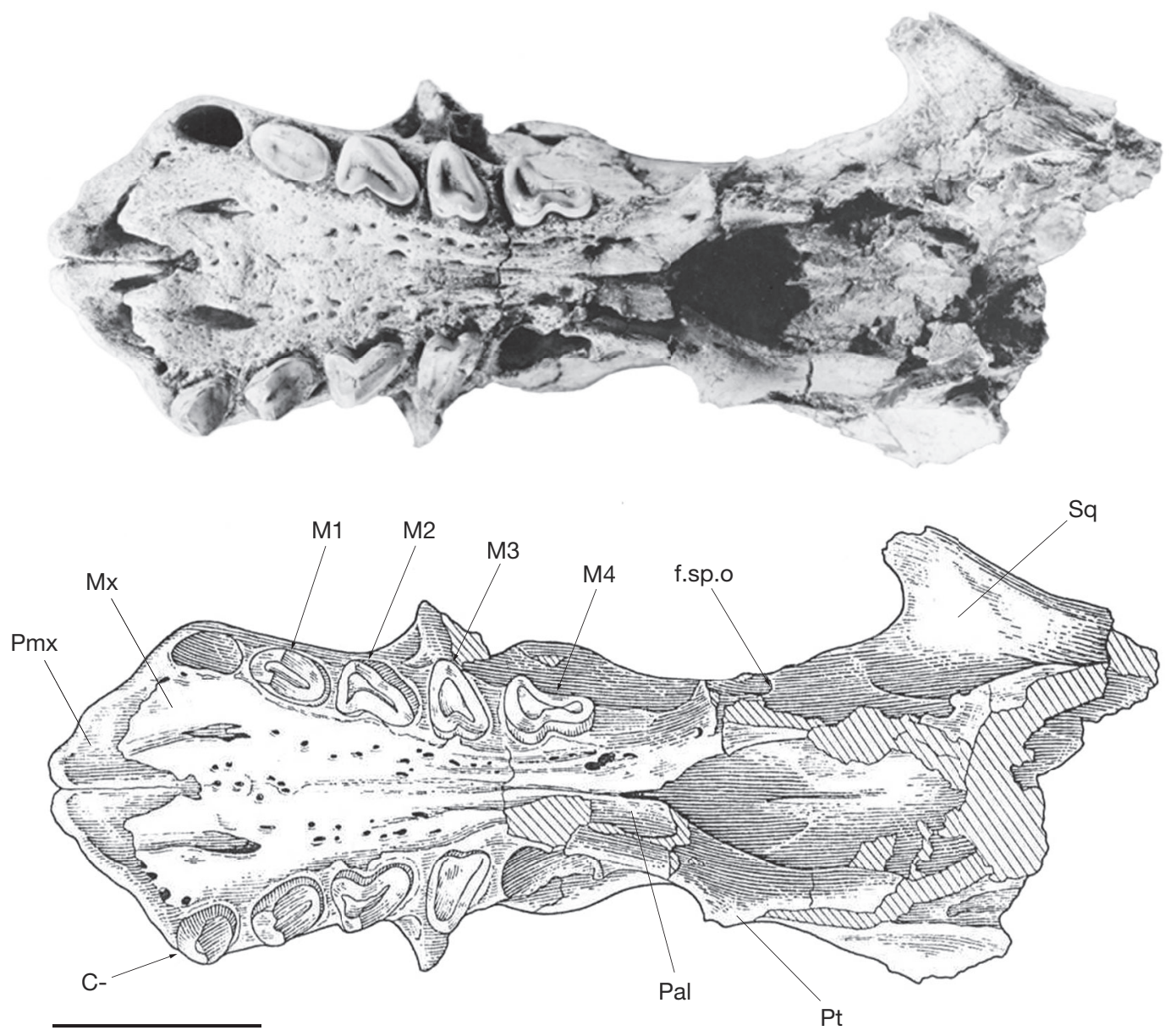

FIG. 3. - Crâne holotype de Pleurolestodon dalenzae n. sp. (MNHN-BOL V 3348) en vue occlusale (antérieure vers la gauche). Abréviations: C-, dent caniniforme supérieure; f.sp.o, fissure sphénorbitaire; M1-M4, dents molariformes supérieures; Mx, maxillaire; Pal, palatin; Pmx, prémaxillaire; Pt, ptérygoïde; Sq, squamosal. Échelle: $5 \mathrm{~cm}$.

En avant et entre les nasaux, se trouvent deux éléments osseux comparables à l'élément unique caractérisant G. wegneri mais toutefois beaucoup moins développé que chez Mylodonopsis ibseni ou Mylodon darwini (Reinhardt 1879; Bargo et al. 2006).

\section{Prémaxillaire (Figs 2-4)}

Contrairement à ce que l'on observe chez Simomylodon uccasamamensis $\mathrm{n}$. gen., n. sp., les prémaxillaires de Pleurolestodon dalenzae n. sp. s'avancent fortement en avant des maxillaires (Fig. 3). En vue occlusale, l'extrémité antérieure du palais a la forme d'un angle émoussé car le bord antérieur des prémaxillaires se projette en avant du niveau de l'alvéole des C-. Les prémaxillaires s'accolent et forment des processus palatins qui s'insèrent dans l'échancrure ménagée à ce niveau sur le bord antérieur des lames palatines des maxillaires (Fig. 3). Entre la suture inter-prémaxillaire et le bord incisif, la surface ventrale du corps du prémaxillaire est concave. Entre le bord antérieur de la lame palatine du maxillaire et le prémaxillaire, la fissure palatine est interrompue par un petit pont osseux situé à l'extrémité antérieure de la lame palatine (Fig. 3). Seule la partie située entre 
ce "pont» et le processus palatin du prémaxillaire a pu être fonctionnelle car, chez Glossotherium wegneri, la partie de la fissure palatine située latéralement par rapport à l'extrémité antérieure de la lame palatine du maxillaire disparaît de l'extension au niveau de la suture entre le prémaxillaire et le maxillaire. Cette disposition a pour effet de renforcer la cohésion entre les deux os. Chez Glossotherium robustum, Paramylodon harlani, Simomylodon uccasamamensis n. gen., n. sp. et sans doute Glossotheridium chapadmalense qui présentent la même disposition que $P$. dalenzae n. sp., prémaxillaire et maxillaire ne sont reliés que par des sutures localisées près de la $\mathrm{C}^{-}$et à l'extrémité postérieure du processus palatin du prémaxillaire, raison pour laquelle de nombreux crânes ont perdu leurs prémaxillaires (Stock 1925: 123). Ceci est encore plus flagrant chez Mylodonopsis ibseni (Cartelle 1991: fig. 3).

Dorsalement, le prémaxillaire porte, au-dessus du pont osseux signalé, le septomaxillaire (Fig. 4), absent ou tout simplement non conservé chez $P$. acutidens. Vers l'extrémité postérieure du processus palatin s'insère le vomer. Conséquence de la forme concave de la face ventrale du corps du prémaxillaire, sa surface dorsale a un profil convexe qui s'abaisse antérieurement jusqu'au bord incisif (Fig. 3).

\section{Maxillaire (Figs 2-4)}

En vue ventrale, le palais s'élargit plus d'arrière en avant que chez $P$. acutidens (FMNH P14495), Mylodonopsis ou Glossotheridium et probablement Pseudoprepotherium, autant que chez Simomylodon n. gen. et Paramylodon et moins que chez Glossotherium (Fig. 3).

À son niveau le plus étroit, c'est-à-dire entre la M3 et la M4, le maxillaire est parcouru longitudinalement par cinq rides épaisses qui lui conferent une légère convexité aussi bien transversale que longitudinale alors qu'il est concave entre C- et M2. Entre la M1 à droite et le septum inter-alvéolaire C-/M1 à gauche, chaque maxillaire porte en son centre un gros foramen prolongé d'un profond sillon vers l'avant (Fig. 3). En arrière de ces foramens s'ouvrent d'autres sillons plus petits et alignés en rangées.
Du côté lingual de chaque alvéole, le maxillaire développe un petit bourrelet présent chez une majorité de paresseux fossiles, peu élevé au niveau de la C-, très fort au niveau de la M2 et qui fusionne avec la ride externe au niveau des M3-M4.

La suture du maxillaire avec le palatin n'est pas visible ventralement et latéralement mais semble présente sur la face interne.

Chez les deux espèces de Pleurolestodon, la base antérieure de l'arcade zygomatique se situe sur les parois latérales du maxillaire à un niveau situé entre le milieu de la M2 et l'extrémité postérieure de la M3 (Figs 2-4), comme chez Pseudoprepotherium; chez Glossotherium (Hoffstetter 1952) elle déborde au-dessus du lobe antérieur de la M4 et chez Mylodon elle est légèrement plus antérieure, entre M1 et M2 (Kraglievich 1934). Chez Mylodonopsis et Paramylodon, elle est située au niveau de l'extrémité antérieure de la M2. Le plancher du canal infra-orbitaire est mince et son niveau ne s'étend qu'entre les M2-M3.

Chez Pleurolestodon dalenzae n. sp., comme chez Simomylodon uccasamamensis n. gen., n. sp., pour chaque plan transversal en avant des arcades zygomatiques, la largeur maximale du museau se situe au niveau du bord alvéolaire. En revanche, chez Glossotherium robustum et $G$. wegneri, cette propriété n'apparaît qu'au niveau de la C- car entre celle-ci et l'arcade zygomatique, la largeur transversale du museau est maximale à mi-hauteur, en avant du toit du canal infra-orbitaire. Il existe également chez $P$. dalenzae n. sp. un léger relief en avant de la racine antérieure de l'arcade zygomatique mais, à ce niveau, la largeur transversale est identique à celle mesurée sur le plan alvéolaire. En fait, comme chez Simomylodon n. gen., le contour du museau en vue ventrale correspond au bord alvéolaire labial (Fig. 3). Ce caractère doit être corrélé avec la moindre dilatation du museau chez l'ensemble des Mylodontinae pré-pléistocènes.

La suture maxillo-nasale est sinueuse uniquement dans ses deux tiers postérieurs (Fig. 4). À son extrémité antérieure, nasal et maxillaire ménagent une petite échancrure entre eux. Le bord dorsal du maxillaire couvre latéralement le bord ven- 


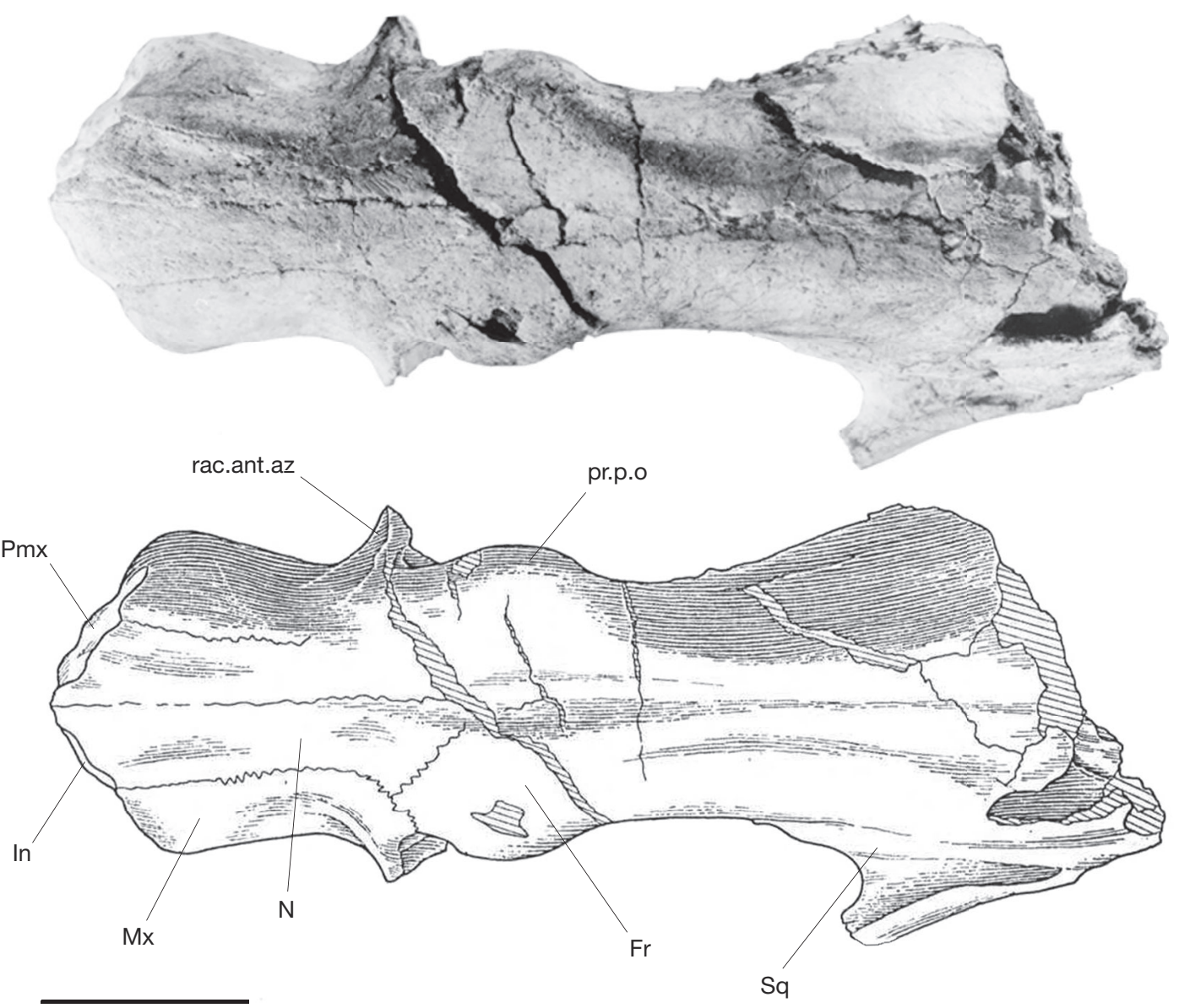

FIG. 4. - Crâne holotype de Pleurolestodon dalenzae n. sp. (MNHN-BOL V 3348) en vue dorsale (antérieure vers la gauche). Abréviations: Fr, frontal; In, internasal; Mx, maxillaire; N, nasal; Pmx, prémaxillaire; pr.p.o, processus postorbitaire; rac.ant.az, racine antérieure de l'arcade zygomatique; Sq, squamosal. Échelle: $5 \mathrm{~cm}$.

trolatéral du nasal, légèrement recourbé dans la cavité nasale. En vue latérale, la suture maxillonasale est de convexité dorsale (Fig. 2). Au-dessus de la base antérieure de l'arcade zygomatique se situe le point "triple» de la suture nasal-maxillaire-frontal (Fig. 2). La suture maxillo-frontale est sinueuse et descend de manière légèrement oblique d'avant en arrière jusqu'au lacrymal (Figs 2 ; 4). La suture maxillo-lacrymale non sinueuse, contourne les parties antérodorsale et antérieure du lacrymal.

En vue ventrale, comme chez P. acutidens, Glossotherium, Simomylodon n. gen. et Glossotheridium, le bord alvéolaire du maxillaire ne s'étend pas en avant de la C- (Fig. 3) contrairement à ce que l'on observe chez Paramylodon, Mylodon et surtout Pseudoprepotherium (Hirschfeld 1985). Par contre, le processus antérieur de la lame palatine du maxillaire s'avance entre le bord latéral du prémaxillaire et le processus palatin de cet os bien en avant du niveau de la C-, comme chez Glossotherium robustum, G. wegneri et Glossotheridium chapadmalense. Cette disposition est sans doute à rapprocher de la projection des prémaxillaires vers l'avant. Chez Simomylodon uccasamamensis n. gen., n. sp., les processus antérieurs des lames 
palatines des maxillaires dépassent à peine le niveau des C- et le bord incisif au niveau des prémaxillaires est légèrement convexe en vue ventrale, ne dessinant aucun prolongement cunéiforme à l'avant du palais.

\section{Palatin (Figs 2; 3)}

Les sutures du palatin avec le maxillaire, le ptérygoïde et le frontal ne sont pas visibles probablement à cause d'une fusion précoce entre ces éléments osseux (Figs 2; 3).

Au-dessus de la racine antérieure de la lame verticale du ptérygoïde s'ouvre le grand foramen sphéno-palatin (foramen orbito-nasale) qui correspond à l'orifice postérieur du conduit palatin (Fig. 2). Ce dernier s'enfonce vers l'avant dans le palatin pour déboucher sur la surface ventrale de celui-ci juste derrière le niveau des M4.

En arrière du foramen sphéno-palatin, sur le même plan frontal, s'ouvre vers l'avant et à l'extrémité postérieure d'une petite fosse, la fissure sphéno-orbitaire pour la branche maxillaire du trijumeau et le nerf optique (Figs 2; 3). Il est sans doute l'homologue des foramina rotundum, lacerum anterius et opticum (Kraglievich 1923, 1925). Sa paroi interne, complexe, doit correspondre au sphéno-orbital, comme chez Mylodon darwini (Guth 1962).

En vue ventrale, l'échancrure post-palatine forme un arc ogival autour des choanes (Fig. 3). L'extrémité postérieure du palais se trouve au niveau du foramen palatin, en arrière du plan transversal passant par les processus post-orbitaires et les M3 mais en avant de ceux passant par le foramen sphéno-palatin ou par la ligne de plus grande convergence des crêtes temporales. Cette disposition rappelle celle trouvée chez les espèces pléistocènes de Glossotherium mais diffère notablement de celle rencontrée chez Glossotheridium dont l'extrémité antérieure de l'échancrure post-palatine se trouve au niveau des processus post-orbitaires des frontaux (Kraglievich 1925).

\section{Ptérygoïde (Figs 2; 3)}

La lame ptérygoïde de Pleurolestodon dalenzae n. sp. diminue d'épaisseur d'arrière en avant comme chez P. acutidens, Glossotheridium et Glossotherium robustum contrairement à Mylodon où son épaisseur est constante. Sa surface médiale est lisse, sa surface latérale porte de forts reliefs.

La suture ptérygo-palatine n'est visible qu'à la base antérieure de la surface médiale de la lame ptérygoïdienne droite (Fig. 3).

Vers l'arrière, sous la partie antérieure de l'apophyse zygomatique du squamosal et sous le plan frontal passant par les foramens sphéno-palatin et sphénoorbitaire, se trouve, comme chez Glossotherium, le foramen ovale, pour la branche mandibulaire du trijumeau.

\section{Internasal (Figs 2; 4)}

Pleurolestodon dalenzae n. sp. possède, en avant des nasaux, un petit prolongement sagittal comparable à celui qui caractérise Glossotherium wegneri (Hoffstetter 1952: pl. 2). Chez ce dernier les nasaux se séparent antérieurement pour former une petite encoche dans laquelle s'insère l'os internasal. Chez Pleurolestodon dalenzae n. sp., les sutures internasonasales sont presque entièrement oblitérées, sauf à leur extrémité antérieure (Fig. 2). Par contre, la suture sagittale des nasaux se prolonge vers l'avant sur la région internasale et se traduit chez $P$. dalenzae n. sp. par un os pair: il vaudrait mieux parler d'os internasaux comme chez Mylodonopsis ibseni (Hirschfeld 1985). Leur extrémité est émoussée, non tronquée et de profil rectiligne comme c'est le cas chez Glossotherium wegneri. Leur surface, dans le prolongement de celle des nasaux, s'incline faiblement en avant de l'extrémité antérieure de ceux-ci comme chez Glossotherium wegneri.

\section{Nasal (Figs 2; 4)}

En vue dorsale et suivant les sutures maxillo-nasales, les nasaux se rétrécissent légèrement d'avant en arrière puis s'élargissent jusqu'au niveau de la base antérieure de l'arcade zygomatique (Fig. 4). À cet endroit, leur largeur est quelque peu supérieure à celle mesurée à leur extrémité antérieure. À partir de cet élargissement postérieur, les nasaux se rétrécissent rapidement vers l'arrière en formant un coin qui s'enfonce entre les frontaux, comme chez l'espèce nord-américaine Paramylodon harlani. Chez Glossotheridium chapadmalense et Glossotherium robustum, ce sont les frontaux qui s'immiscent de manière cunéiforme entre les nasaux (Kraglievich 1925). L'extrémité antérieure des nasaux 
dessine une accolade dont la pointe, dans le plan sagittal, correspond aux os internasaux. Les sutures internaso-nasales sont indistinctes vers l'arrière mais sont parallèles l'une à l'autre et à la suture sagittale dans leur partie antérieure. Chez G. wegneri, ces sutures convergent vers l'arrière, la limite antérieure des nasaux formant à ce niveau une encoche triangulaire aux bords convexes.

En vue antérieure, les limites latérales des nasaux se recourbent légèrement dans la cavité nasale et ne touchent pas le bord supérieur des maxillaires qui s'accolent un peu plus haut.

\section{Région fronto-pariétale (Figs 2; 4)}

Les sutures des frontaux avec les nasaux, les maxillaires et les lacrymaux sont les seules visibles. Les sutures fronto-maxillaires, très sinueuses entre les nasaux et les lacrymaux, sont indistinctes sous cet os (Figs $2 ; 4)$.

Le processus post-orbitaire est plus développé que chez Glossotherium robustum ou $G$. wegneri et moins saillant que chez Mylodon darwini. Chez Paramylodon harlani (Stock 1925), Glossotheridium chapadmalense et surtout Pleurolestodon acutidens ce processus est peu proéminent (Kraglievich 1921, 1925). Chez P. dalenzae n. sp., il forme un épais relief dirigé antéropostérieurement (Fig. 4). La ride post-orbitaire, qui descend d'avant en arrière comme chez les espèces pléistocènes, est moins marquée que chez ces dernières et à peine perceptible. Entre les processus post-orbitaires se situe la dépression frontale par laquelle la ligne sagittale s'abaisse de la région fronto-pariétale à la région nasale (Fig. 2). Cette "démarcation» est d'autant mieux marquée que cette zone est transversalement plane, alors qu'elle est fortement convexe chez Glossotherium.

Vers l'arrière, les lignes temporales convergent plus rapidement que chez Glossotherium (Fig. 4). Elles délimitent entre elles une surface proportionnellement plus étroite. Le plan transversal de leurs points de convergence maximale passe aussi entre les foramens sphéno-orbitaires comme chez $G$. robustum, alors que chez $G$. wegneri, ce même plan passe par la racine postérieure des arcades zygomatiques, un peu en arrière des foramens ovales.

\section{Genre Simomylodon n. gen.}

ESPÈCE TYPE. - Simomylodon uccasamamensis n. sp., seule espèce du genre.

Diagnose. - La même que pour l'espèce.

ÉtYMologie. - Le nom du genre dérive de l'adjectif grec simos, - $\hat{e}$, -on signifiant «canard», en référence aux prémaxillaires formant, sous l'ouverture nasale, un bord incisif quasiment droit par opposition à ce que l'on observe chez Glossotherium ainsi qu'à l'absence, en avant des nasaux, de la proéminence due au développement de l'os internasal que possède $G$. wegneri. Le second membre du composé rappelle la proche parenté de l'animal avec le genre Mylodon.

Simomylodon uccasamamensis n. gen., n. sp. (Figs 5-19; Tableaux 2-4)

"Simotherium uccasamamense» - Saint-André 1994: 184-228, figs 19, 20, pls 14-22 (unpublished).

HolotyPe. - GB 078 (Figs 5; 6; Tableau 2), portion antérieure d'un crâne sans dents provenant du gisement de Viscachani (Fig. 1), découvert par Carlos Villarroel.

ParatyPe. - MNHN-BOL V 3321 (Fig. 7), maxillaires et prémaxillaires provenant du gisement d'Ayo Ayo, découverts en 1989 au cours d'une mission conjointe de l'IFEA et du MNHN-BOL.

Localité type. - Site de Viscachani (Fig. 1).

Niveau TYPE. - Ce matériel, collecté dans les sédiments de la Formation Umala entre la Toba 76 (5,4 Ma) et le tuf Ayo Ayo (2,8 Ma), date du Pliocène inférieur et moyen (Montehermoséen-Chapadmalaléen).

ÉTYMologie. - Le nom de l'espèce correspond à la latinisation d'un composé descriptif sanskrit transcrivant le terme espagnol "Altiplano» et qui est formé à partir de la racine $u c c a$ - "élevé, haut» et du substantif neutre $\operatorname{sama}(m)$ "plaine, étendue». Le suffixe latin -ense est un suffixe dénominal donnant valeur de locatif à l'ensemble qui signifie «de l'Altiplano».

Autre MATÉRIEL EXAMINÉ. - Spécimens provenant du gisement de Viscachani et attribués à Simomylodon uccasamamensis n. gen., n. sp. : condyle occipital (MNHN VIZ 18); fragment d'hémimandibule gauche avec les trois molaires m1-m3 (MNHN VIZ 1); fragments de dents dont une C-droite (MNHN VIZ 35); extrémité ventrale (avec partie de cavité glénoïde) d'omoplate (MNHN VIZ 4); extrémité distale incomplète d'humérus gauche 

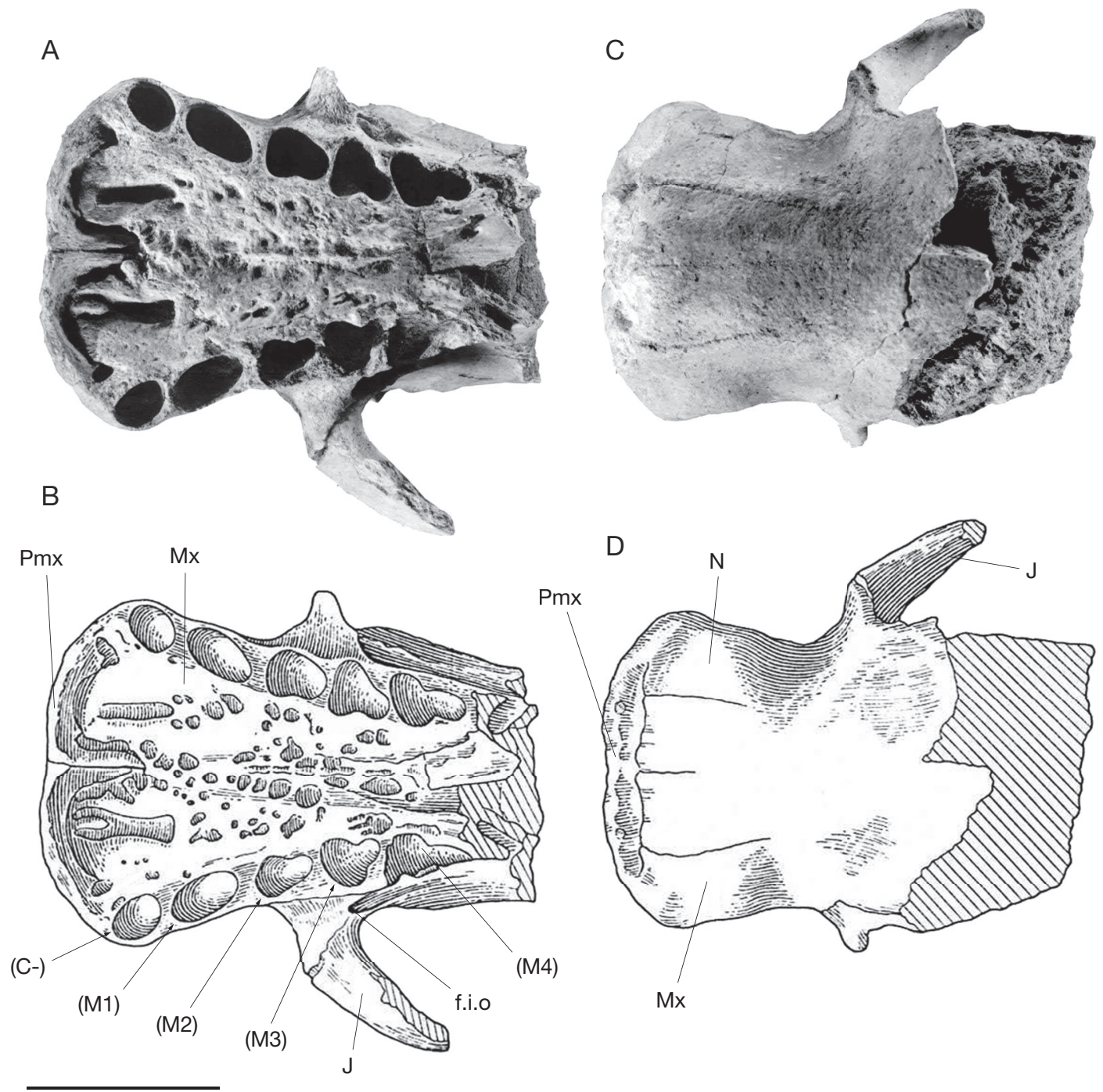

FIG. 5. - Avant du crâne holotype de Simomylodon uccasamamensis n. gen., n. sp. (GB 078): A, B, vue occlusale; C, D, vue dorsale (antérieure vers la gauche). Abréviations: C-, alvéole de dent caniniforme supérieure; f.i.o, foramen infra-orbitaire; J, jugal; M1-M4, alvéole de dent molariforme supérieure; Mx, maxillaire; $\mathbf{N}$, nasal; Pmx, prémaxillaire. Échelle: $5 \mathrm{~cm}$.

(MNHN VIZ 3); extrémité proximale de radius gauche dont la diaphyse est incomplète (MNHN VIZ 16); semilunaire gauche (MNHN VIZ 17); métacarpien I droit (MNHN VIZ 32); métacarpien III droit non soudé au magnum (MNHN VIZ 27, Fig. 14); métacarpien III gauche non soudé au magnum (MNHN VIZ 5); métacarpien IV gauche sans extrémité distale (MNHN VIZ 33); métacarpien IV droit (MNHN VIZ 24); articulation distale de tibia gauche (MNHN VIZ 22); métatarsien IV droit sans extrémité distale (MNHN VIZ 28); fragment de côte (MNHN VIZ 30); axis incomplet (MNHN VIZ 31); corps de vertèbre dorsale (MNHN VIZ 7); corps de vertèbre (MNHN VIZ 9); centre de vertèbre dorsale (MNHN VIZ 13 et 14); centre de vertèbre caudale (MNHN VIZ 8 et 34); centre de vertèbre caudale avec apophyses transverses (MNHN VIZ 23); vertèbre caudale (MNHN VIZ 2, 26 et 29).

Spécimens provenant du gisement d'Ayo Ayo et attribués à Simomylodon uccasamamensis n. gen., n. sp.: fragment de maxillaire (MNHN AYO 135); fragment antéro-laté- 

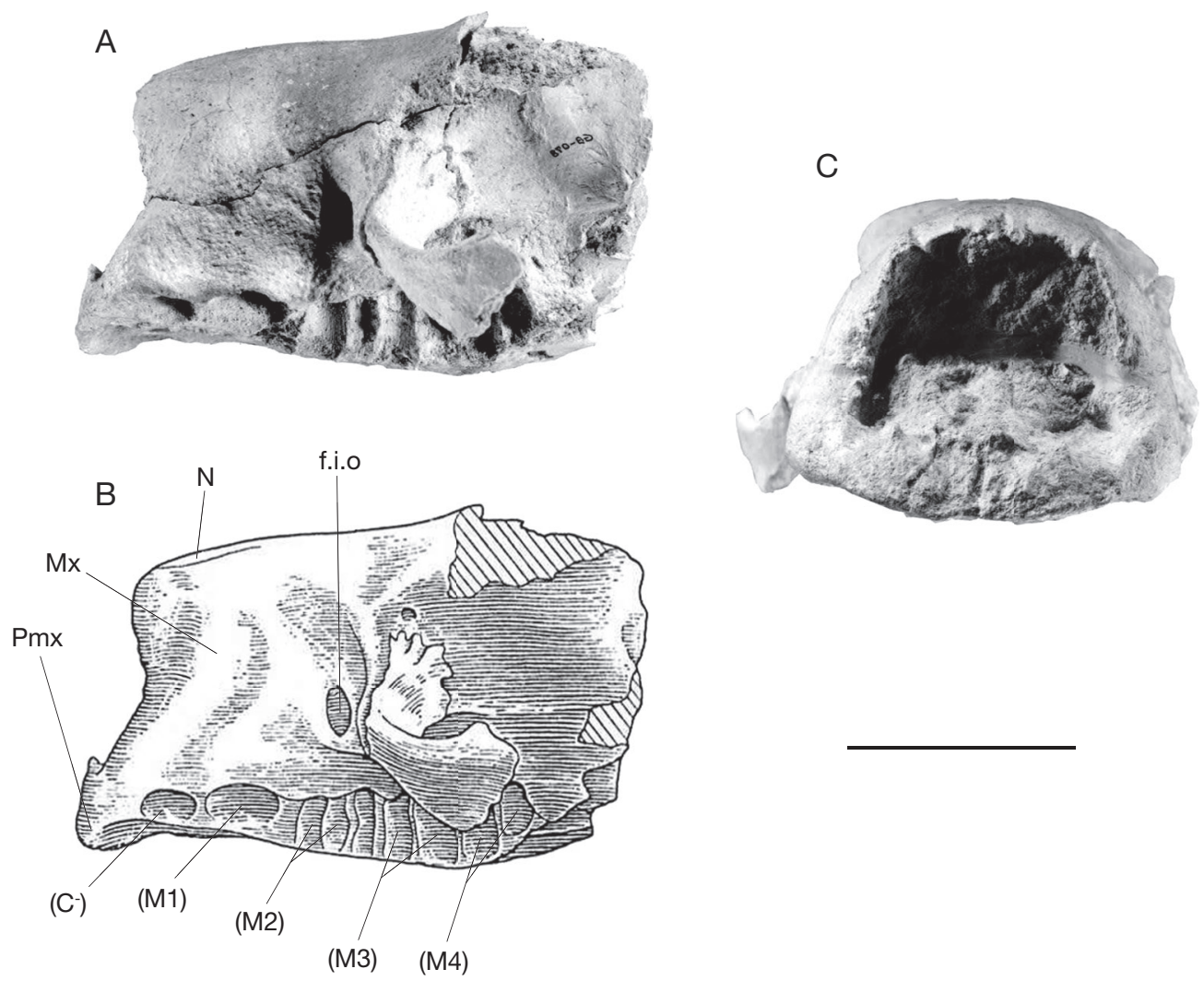

FIG. 6. - Avant du crâne holotype de Simomylodon uccasamamensis n. gen., n. sp. (GB 078): A, B, vue latérale (antérieure vers la gauche, retourné par rapport à l'original); C, vue antérieure (dorsale vers le haut). Abréviations: C-, alvéole de dent caniniforme supérieure; f.i.o, foramen infra-orbitaire; M1-M4, alvéoles de dents molariformes supérieures; Mx, maxillaire; N, nasal; Pmx, prémaxillaire. Échelle: $5 \mathrm{~cm}$

ral de maxillaire droit comprenant des parties des parois alvéolaires des C- et M1 (MNHN AYO 138); fragments de C- gauche, M1 gauche, M3 gauche et $\mathrm{m} 1$ droite d'un même individu (MNHN AYO 188); m3 droite et m2 droite (MNHN AYO 164); hémimandibule droite (MNHN AYO 185); partie postérieure de la branche horizontale d'une hémimandibule droite (MNHN AYO 181); symphyse mandibulaire (MNHN AYO 186); branche horizontale d'une hémimandibule droite de petite taille (MNHN AYO 165) ; symphyse mandibulaire (MNHN AYO 208); fragment de mandibule (MNHN AYO 209); fragment de mandibule (MNHN AYO 133); humérus droit sans extrémité proximale (MNHN AYO 110, Fig. 10); moitié distale sans épiphyse d'un humérus droit appartenant à un individu juvénile (MNHN AYO 184) ; extrémité distale d'un humérus gauche (MNHNBOL V 3303); moitié distale d'un humérus gauche (MNHN AYO 222); humérus gauche (MNHN AYO 102); région articulaire proximale d'un humérus gau- che (MNHN AYO 227); région articulaire distale d'un humérus gauche (MNHN AYO 228); région articulaire distale d'un humérus droit (MNHN AYO 229); tête d'humérus droit (MNHN AYO 121); radius gauche, scaphoïde gauche (Fig. 12A, B), semi-lunaires droit et gauche (Fig. 12C, D), trapézoïde gauche (Fig. 12E-G), métacarpien III-magnum gauches soudés, métacarpien II gauche (Fig. 13), une phalange intermédiaire, quatre phalanges unguéales, une phalange nodule, fragment proximal de côte droite, trois vertèbres dorsales (une complète, une avec la base des arcs neuraux, une centre), un corps de vertèbre cervicale et une sternèbre (MNHN AYO 180); radius gauche (MNHN AYO 180, Fig. 11); métacarpien III-magnum gauches soudés (Fig. 15), phalange proximale gauche d'un même doigt (MNHN AYO 179); extrémité proximale de radius droit (MNHN AYO 126); radius droit sans épiphyse proximale (MNHN AYO $178)$; radius gauche sans épiphyse proximale (MNHN AYO 223); métacarpien II droit (MNHN AYO 190); 
TABLEAU 2. - Principales dimensions (en $\mathrm{mm}$ ) des restes crâniens et mandibulaires attribués à Simomylodon uccasamamensis n. gen., n. sp.

\begin{tabular}{|c|c|c|}
\hline & MNHN-BOL V 3296 & GB 078 \\
\hline Largeur entre les C- & 72,9 & 62 \\
\hline Largeur entre les $\mathrm{M} 1$ & 59,2 & 48 \\
\hline Largeur entre les M2 & 43,7 & 39,4 \\
\hline Largeur entre les M3 & 34,4 & 29 \\
\hline Largeur entre les M4 & 32,6 & 26,4 \\
\hline Longueur de l'alvéole de la C- & $\sim 16$ & 14 \\
\hline Largeur maximale de la C- & $\sim 16$ & 11 \\
\hline Longueur de l'alvéole de la M1 & 29,7 & 21,8 \\
\hline Largeur maximale de la M1 & 13,2 & 11 \\
\hline Longueur de l'alvéole de la M2 & 20,7 & 16,4 \\
\hline Longueur linguale de la M2 & 16,8 & 16,2 \\
\hline Largeur maximale de la M2 & 13,6 & 12,4 \\
\hline Longueur maximale de l'alvéole de la M3 & 22,4 & 18,4 \\
\hline Longueur, face linguale, de l'alvéole de la M3 & 16,2 & 16,1 \\
\hline Largeur maximale de la M3 & 15,7 & 14,8 \\
\hline Longueur de l'alvéole de la M4 & 20,0 & 21,8 \\
\hline Largeur maximale du lobe antérieur de la M4 & 16,3 & 14,5 \\
\hline Largeur maximale du lobe postérieur de la M4 & 8,4 & 8,0 \\
\hline Largeur minimale de l'isthme entre les lobes de la M4 & 7,2 & 7,1 \\
\hline Longueur C--M4 & 104 & 90,0 \\
\hline Longueur M1-M2 & 86,4 & 74,8 \\
\hline Longueur M2-M4 & 54,3 & 53,8 \\
\hline Longueur M3-M4 & 35,2 & 35,5 \\
\hline $\begin{array}{l}\text { Longueur du palais, du bord antérieur du maxillaire au sommet } \\
\text { de l'échancrure postpalatine }\end{array}$ & - & 93 \\
\hline Largeur maximale du museau sur le renflement maxillaire & - & 89,3 \\
\hline Distance entre les bords alvéolaires externes des C- & - & 86,4 \\
\hline Distance minimale entre les M4 et le sommet de l'échancrure postpalatine & - & 19 \\
\hline Largeur minimale du museau en avant des apophyses zygomatiques & - & 71,3 \\
\hline Longueur de l'extrémité antérieure de la symphyse au condyle & 222,5 & - \\
\hline Longueur de l'extrémité antérieure de la symphyse au processus angulaire & 243,5 & - \\
\hline Longueur de la symphyse & 63,0 & - \\
\hline Largeur du bord incisif & 34,9 & - \\
\hline Hauteur de la branche horizontale sous la C- & 56,4 & - \\
\hline Hauteur de la branche horizontale sous la m1 & 54,0 & - \\
\hline Hauteur de la branche horizontale sous la m2 & 54,7 & - \\
\hline Hauteur de la branche horizontale sous l'isthme de la m3 & 55,0 & - \\
\hline
\end{tabular}

MNHN-BOL V 3296 MNHN AYO 164

Longueur de la C-

Largeur de la C-

Longueur de la m1

Largeur de la m1

Longueur antéropostérieure de la m2

Diamètre depuis l'angle antéro-externe jusqu'à l'angle postéro-lingual

Largeur de la m2

Longueur de la m3

Largeur du lobe antérieur de la m3

Largeur du lobe postérieur de la m3

\section{6,5}

11,4

14,6

9,8

9,7

17,4

13,4

31,6

16,2

13,5

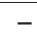

$-$

$-$

$-$

11,0

17,0

14,5

29,8

15,6

12,5 métacarpien II gauche (MNHN-BOL V 3318); fémur droit (MNHN-BOL V 3299, Fig. 16); diaphyse de fémur gauche sans épiphyses (MNHN AYO 166); moitié distale de fémur gauche (MNHN-BOL V 3301); tête de fémur droit (MNHN AYO 129); fémur droit sans tête ni extrémité distale (MNHN AYO 167); fémur droit sans épiphyses (MNHN AYO 197); tiers distal de fémur droit (MNHN AYO 168); extrémité articulaire distale de 
fémur gauche avec ectépicondyle (MNHN AYO 169); diaphyse de fémur gauche (MNHN AYO 196); rotule gauche (MNHN-BOL V 3300, Fig. 17); tibia gauche (Fig. 18), extrémité proximale de tibia droit (MNHNBOL V 3305); moitié distale de tibia gauche (MNHN AYO 201); extrémité distale de tibia droit (MNHN AYO 200); phalanges proximale, intermédiaire et distale d'un même doigt d'un même individu (MNHN-BOL V 3313); semi-lunaire gauche (MNHN AYO 111); astragale gauche (MNHN AYO 182, Fig. 19); cuboïde droit (MNHN AYO 128); fragment de côte (MNHN AYO 132); fragments de deux côtes (MNHN AYO 112); fragment proximal de côte (MNHN AYO 113); fragments de sacrum avec cavités cotyloïdes et vertèbre lombaire incomplète (MNHN AYO 148); axis incomplet (MNHN AYO 170); vertèbre cervicale (MNHN AYO 156); centre de vertèbre (MNHN AYO 171); vertèbre dorsale (MNHN AYO 198); vertèbre dorsale postérieure (MNHN AYO 187); corps de vertèbre dorsale (MNHN AYO 205); vertèbre caudale incomplète (MNHN AYO 207); vertèbre caudale (MNHN AYO 217); centre de vertèbre dorsale (MNHN AYO 218); centre de vertèbre caudale (MNHN AYO 131); vertèbre caudale (MNHN AYO 219); vertèbre caudale (MNHN AYO 220); vertèbre caudale (MNHN AYO 210); vertèbre caudale (MNHN AYO 206); centre de vertèbre caudale (MNHN AYO 172); vertèbre caudale complète (MNHN AYO 183).

Spécimens provenant du gisement de Pomata et attribués à Simomylodon uccasamamensis n. gen., n. sp.: hémimandibule gauche (MNHN-BOLV 3296); partie antérieure avec $\mathrm{C}_{-}-\mathrm{m} 2$ d'une hémimandibule gauche (MNHN-BOL V 3298); humérus gauche sans épiphyses d'un individu juvénile (MNHN POM 25); extrémité distale de métacarpien III gauche (MNHN POM 79); tibia droit (MNHN POM 62); astragale droit (MNHN POM 64); astragale gauche incomplet (MNHN POM 63); cuboïde droit (MNHN POM 66); atlas (MNHN POM 57); vertèbre cervicale incomplète (MNHN POM 20); vertèbre cervicale incomplète (MNHN POM 60); centre de vertèbre cervicale (MNHN POM 61); vertèbre lombaire (MNHN POM 58); vertèbre caudale (MNHN POM 26); vertèbre caudale incomplète (MNHN POM 56).

DiAgnose. - Mylodontinae de plus petite taille (voir Tableaux 2-4) que Glossotheridium (la longueur du palais ne correspond qu'à un tiers de celle de ce dernier) montrant un fort élargissement du museau vers l'avant; séries dentaires divergeant vers l'avant et ne montrant aucun diastème entre les alvéoles des dents supérieures. Prémaxillaires s'abaissant obliquement vers l'avant, formant un bord incisif de contour légèrement et continûment convexe en vue latérale, ne présentant pas l'extension qui, dans leur région sagittale, confere chez Glossotherium un contour en accolade; le bord alvéolaire des maxillaires

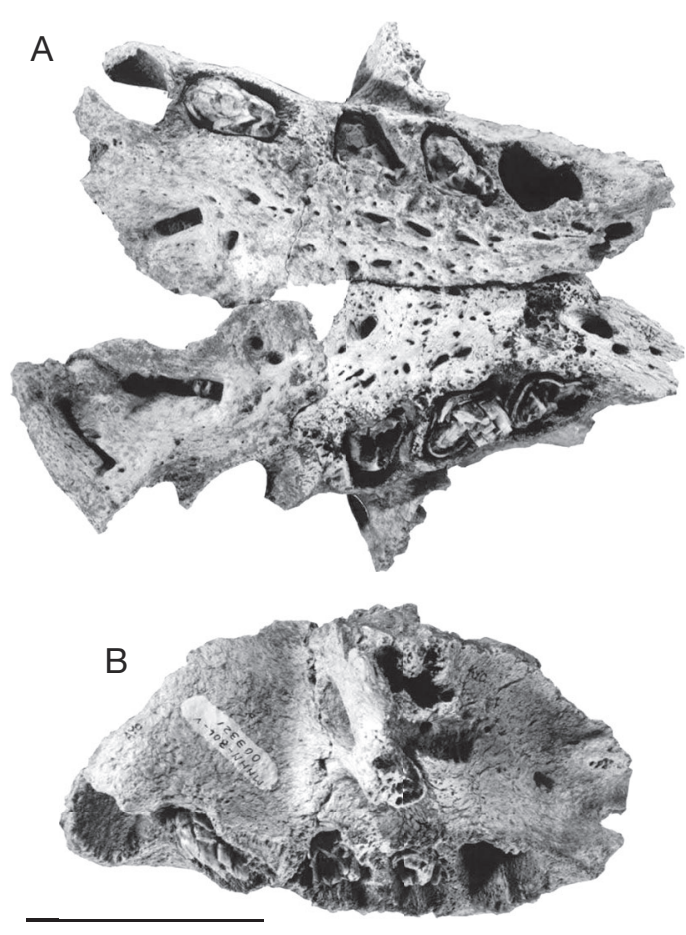

FIG. 7. - Maxillaires et prémaxillaires attribués à Simomylodon uccasamamensis n. gen., n. sp. (MNHN-BOL V 3321): A, vue occlusale; B, vue latérale droite, antérieur vers la gauche. Échelle: $5 \mathrm{~cm}$.

ne se prolonge pas en avant des $\mathrm{C}^{-}$; le processus antérieur des lames palatines des maxillaires, engagé entre le corps du prémaxillaire et le processus médial de celui-ci et arrondi à son extrémité, dépasse légèrement le plan transversal passant par le bord antérieur des alvéoles des C- et il est nettement moins proéminent que chez Glossotherium et Glossotheridium; les nasaux ne dessinent pas de prolongement antérieur près de leur suture sagittale; il n'existe pas d'os internasal et par rapport aux genres cités, le crâne de Simomylodon n. gen. est camus (grec: simos); C_ triangulaire en section, présentant un double biseau, située dans le même axe principal antéropostérieur de la série des dents molariformes et proportionnellement beaucoup plus grande (que les autres dents) que chez la majorité des autres Mylodontinae; m2 de section grossièrement rectangulaire; face antérolinguale de la m3 concave et non convexe comme chez Kiyumylodon; lobe postérieur de la $\mathrm{m} 3$ nettement moins étiré antéropostérieurement que chez Glossotherium; en vue latérale la ramus du dentaire est nettement moins haute que chez Kiyumylodon et les bords dorsal et ventral ne se biseautent pas vers l'avant comme chez Glossotherium; «bec" de la mandibule en forme de "pelle» et non en forme de «bec de cruche». 
TABleau 3. - Principales dimensions (en $\mathrm{mm}$ ) des éléments du membre antérieur de Simomylodon uccasamamensis n. gen., n. sp.

\begin{tabular}{|c|c|c|c|}
\hline Humérus & $\begin{array}{l}\text { MNHN } \\
\text { AYO } 102\end{array}$ & $\begin{array}{c}\text { MNHN } \\
\text { AYO } 110\end{array}$ & $\begin{array}{l}\text { MNHN-BOL } \\
\text { V } 3303\end{array}$ \\
\hline Longueur depuis la tête jusqu'au centre de la surface articulaire distale & 245,5 & - & - \\
\hline $\begin{array}{l}\text { Longueur depuis l'extrémité proximale du grand tubercule jusqu'à } \\
\text { l'extrémité de la surface pour le radius }\end{array}$ & 261,5 & - & - \\
\hline Diamètre antéro-postérieur maximal de la tête & 60,5 & - & - \\
\hline Largeur maximale de l'extrémité proximale & 98,9 & - & - \\
\hline $\begin{array}{l}\text { Distance depuis le grand tubercule jusqu'à l'extrémité distale de la crête } \\
\text { deltoïdienne }\end{array}$ & 159,1 & - & - \\
\hline $\begin{array}{l}\text { Largeur maximale de la diaphyse au niveau du bord latéral de la crête } \\
\text { deltoïdienne }\end{array}$ & 57,5 & 57,5 & - \\
\hline $\begin{array}{l}\text { Largeur maximale de l'extrémité distale (mesuré perpendiculairement } \\
\text { au bord supérieur de l'ectépicondyle) }\end{array}$ & - & 120,5 & 152,5 \\
\hline Largeur de la surface d'articulation distale & 76,2 & 73,5 & - \\
\hline \multicolumn{3}{|l|}{ Radius } & $\begin{array}{c}\text { MNHN } \\
\text { AYO } 180\end{array}$ \\
\hline \multicolumn{3}{|l|}{$\begin{array}{l}\text { Longueur maximale } \\
\text { Diamètre maximal de l'épiphyse proximale } \\
\text { Diamètre minimal de l'épiphyse proximale } \\
\text { Diamètre maximal de l'épiphyse distale } \\
\text { Diamètre minimal de l'épiphyse distale }\end{array}$} & $\begin{array}{l}19,4 \\
36,0 \\
29,0 \\
66,5 \\
50,0\end{array}$ \\
\hline \multicolumn{3}{|l|}{ Pyramidal } & $\begin{array}{l}\text { MNHN } \\
\text { AYO } 109\end{array}$ \\
\hline \multicolumn{3}{|c|}{$\begin{array}{l}\text { Diamètre dorsopalmaire maximal } \\
\text { Diamètre médiolatéral maximal } \\
\text { Diamètre médiolatéral maximal de la surface pour l'ulna } \\
\text { Diamètre transversal maximal (dorsomédial latéropalmaire) de la surface pour l'unciforme } \\
\text { Diamètre antéropostérieur }\end{array}$} & $\begin{array}{l}33,7 \\
30,7 \\
25,0 \\
36,4 \\
31,2\end{array}$ \\
\hline \multicolumn{3}{|l|}{ Semi-lunaire } & $\begin{array}{c}\text { MNHN } \\
\text { AYO } 180\end{array}$ \\
\hline \multicolumn{3}{|c|}{$\begin{array}{l}\text { Épaisseur maximale entre la facette pour le radius et le centre de la facette pour l'unciforme } \\
\text { Hauteur de la surface pour le radius } \\
\text { Largeur maximale de la surface articulaire distale depuis la facette pour le pyramidal à celle } \\
\text { pour le scaphoïde }\end{array}$} & $\begin{array}{l}29,8 \\
39,5 \\
35,3\end{array}$ \\
\hline
\end{tabular}

\begin{tabular}{lc} 
Scaphoïde & $\begin{array}{c}\text { MNHN } \\
\text { AYO 180 }\end{array}$ \\
\hline $\begin{array}{l}\text { Épaisseur maximale depuis la double facette pour le semi-lunaire et le radius à celle } \\
\text { pour le trapézoïde }\end{array}$ & 51,9 \\
Hauteur maximale de la surface pour le trapèze à celle pour le semi-lunaire & \\
Largeur maximale au niveau des facettes pour le magnum et le trapézoïde & 54,3 \\
& 41,9 \\
\hline & MNHN \\
Trapézoïde & AYO 180 \\
\hline Largeur maximale au niveau de la facette pour le scaphoïde & 24,1 \\
Hauteur maximale au niveau de la facette pour le métacarpien II & 22,4 \\
\hline & MNHN \\
Métacarpien II & AYO 180 \\
\hline Longueur maximale & 55,6 \\
Largeur au milieu du corps & 20,4 \\
Hauteur au milieu du corps & 19,8 \\
Hauteur de la carène & 28,8 \\
Largeur maximale de la surface articulaire distale & 19,2 \\
Largeur maximale de l'extrémité distale & 24,7 \\
\hline
\end{tabular}


TABleau 3. - Suite.

\begin{tabular}{|c|c|c|}
\hline Métacarpien III-magnum (soudés) & $\begin{array}{c}\text { MNHN } \\
\text { AYO } 180\end{array}$ & $\begin{array}{c}\text { MNHN } \\
\text { AYO } 179\end{array}$ \\
\hline Longueur maximale & 88,3 & 93,5 \\
\hline Hauteur du corps de la partie métapodiale & 22,9 & 25,0 \\
\hline Largeur du corps de la partie métapodiale & 23,8 & 21,0 \\
\hline Hauteur de la carène de la partie métapodiale & 34,0 & 35,0 \\
\hline Largeur maximale de l'articulation distale & 27,5 & 29,0 \\
\hline Largeur maximale de l'extrémité distale & 29,1 & 29,0 \\
\hline Largeur maximale entre les facettes pour les Mc IV et II & 45,1 & 46,8 \\
\hline Hauteur maximale au niveau de la suture & 36,0 & 36,0 \\
\hline Largeur de la partie basipodiale entre les facettes pour l'unciforme et inférieure pour le Mc II & 24,0 & 29,6 \\
\hline \multirow[t]{2}{*}{ Hauteur de la limite entre le scaphoïde et le semi-lunaire } & 22,8 & 23,2 \\
\hline & & $\begin{array}{l}\text { MNHN } \\
\text { VIZ } 27\end{array}$ \\
\hline Longueur maximale & & 67,3 \\
\hline Hauteur au milieu du corps & & 23,4 \\
\hline Largeur au milieu du corps & & 23,1 \\
\hline Hauteur de la carène & & 33,7 \\
\hline Largeur maximale de la surface articulaire distale & & 28,5 \\
\hline Largeur maximale de l'extrémité distale & & 29,2 \\
\hline Largeur proximale maximale & & 42,3 \\
\hline \multirow[t]{2}{*}{ Hauteur proximale maximale } & & 36,4 \\
\hline & & MNHN \\
\hline Métacarpien IV & & VIZ 33 \\
\hline Hauteur du corps & & 22,6 \\
\hline Largeur du corps & & 13,3 \\
\hline Largeur proximale maximale entre les facettes pour les $\mathrm{Mc}$ III et $\mathrm{V}$ & & 27,8 \\
\hline Hauteur proximale maximale & & 35,0 \\
\hline
\end{tabular}

\section{Description (Figs 5-19; TABleauX 2-4)}

Dents supérieures (Figs $5 A, B ; 6 A, B ; 7 A$; Tableau 2) Sur le spécimen MNHN-BOL V 3321 (Fig. 7), les alvéoles des $\mathrm{C}^{-}$et $\mathrm{M} 1$ ne sont pas séparées par le diastème que l'on retrouve par exemple chez les espèces du genre Glossotherium, ce qui aurait tendance à rapprocher Simomylodon n. gen. de la majorité des Mylodontinae comme Glossotheridium, Paramylodon, Pseudoprepotherium, Mylodonopsis et Pleurolestodon.

Comme chez les espèces de Pleurolestodon et Pseudoprepotherium, la série dentaire forme en vue occlusale une très légère courbure (Figs 5A, B ; 7A) alors que chez Glossotherium et Urumacotherium elle parait nettement plus curviligne en raison de la position fortement antéro-externe de la C- par rapport aux autres dents. La forme Pléistocène Mylodon a perdu la dent, probablement caniniforme, la plus antérieure. Cependant la position des quatre dents conservées traduirait que Mylodon se rapproche plus de l'implantation subrectiligne observable chez Pleurolestodon.

En regard des alvéoles dentaires du spécimen MNHN-BOL V 3321, les dents devraient ressembler à celles de Glossotherium (Fig. 7) et présenter une morphologie différente de celles de Mylodonopsis ou Urumacotherium.

L'alvéole de la C- est en position antéro-externe, plus petite que celle de la M1 et la différence de taille entre les deux dents les plus antérieures est plus marquée que chez Glossotherium robustum et Pleurolestodon dalenzae n. sp. et moins que chez Mylodonopsis ibseni ou Paramylodon harlani. La circonférence de cette alvéole est ellipticotriangulaire à ovale (Figs 5A, B ; 7A). Son angle antérieur n'est pas observable sur le matériel à disposition. L'angle postérolabial est plus fortement arrondi que l'angle postérolingual. Un fragment d'une C- (MNHN AYO 188) présente une face linguale plane et plus large que les autres faces. Sur cette dent, la face 
TABLEAU 4. - Principales dimensions (en mm) des éléments du membre postérieur de Simomylodon uccasamamensis n. gen., $\mathrm{n}$. sp.

Fémur

Longueur depuis la tête jusqu'à l'extrémité condylienne médiale

Longueur depuis grand trochanter jusqu'à la surface inférieure du condyle latéral

Largeur depuis la surface médiale de la tête jusqu'à la surface latérale du grand trochanter

Diamètre de la base de la tête

Diamètre médiolatéral minimal de la diaphyse

Largeur épicondylienne maximale

Largeur au niveau des condyles

Largeur maximale du condyle interne

Hauteur du condyle interne

Rotule

Longueur proximodistale maximale

Largeur latéromédiale maximale

Épaisseur antéropostérieure maximale

Longueur proximodistale maximale de la surface pour le fémur

Largeur maximale de la surface pour le fémur

Tibia

Longueur depuis l'éminence interglénoïdienne de l'extrémité proximale jusqu'au centre de l'éminence astragalienne

Longueur depuis la facette glénoïdienne médiale jusqu'à la maléole interne

Largeur maximale de l'extrémité proximale

Largeur maximale de la surface glénoïdienne médiale

Largeur maximale de la surface glénoïdienne latérale

Largeur maximale de la facette fibulaire proximale

Diamètre médiolatéral minimal de la diaphyse

Diamètre antéropostérieur minimal de la diaphyse

Diamètre médiolatéral maximal de l'extrémité distale

Diamètre antéropostérieur maximal de l'extrémité distale

Astragale

Longueur maximale

Longueur du condyle latéral

Hauteur depuis le centre de la facette ectale pour le calcanéum jusqu'au sommet du condyle médial

Longueur de la tête articulaire entre le sulcus tali et le bord antérolatéral

Hauteur depuis la facette ectale pour le calcanéum jusqu'à la base postérieure du condyle médial

Longueur depuis le bord antérieur du condyle médial jusqu'au bord postérieur du condyle latéral

Cuboïde

MNHN AYO 128

Diamètre proximo-distal à travers le milieu de l'os

Diamètre entre le centre des facettes pour le calcanéum et le MC III

27,1

38,0

42,0

Métatarsien IV

MNHN VIZ 28

Hauteur dorsopalmaire maximale de l'extrémité proximale

39,3

Largeur médiolatérale maximale de l'extrémité proximale
324,5

318,0

142,3

87,8

125,5

100,8

41,3

50,6

88,1

60,5

40,5

44,0

59,1

MNHN-BOL V 3299

177,0

176,5

102,3

67,5

37,0

46,9

29,5

80,0

63,4

83,4

73,9

66,1

43,5

30,4

65,2 postérolabiale est plane et la face antérolabiale est nettement arrondie. Elles sont d'égale largeur et presque perpendiculaires entre elles (Tableau 2). Enfin l'angle labial est très arrondi. Comme chez
Glossotherium, cette dent est fortement arquée et caniniforme comme chez Glossotherium et Glossotheridium. Sa surface d'usure est un biseau simple de normale dirigée vers l'arrière. La paroi séparant 
les alvéoles de la C- et de la M1 est de faible épaisseur (Figs 5A, B; 7A).

La section de la M1 est allongée antéropostérieurement et son grand axe est dirigé antérolabialement à postérolingualement (Figs 5A, B; 7A) comme chez Mylodonopsis. La face linguale de son alvéole est très légèrement convexe. Chez le paratype (MNHN-BOL V3321), sa configuration et celle de son alvéole laissent supposer que son fût devait sortir obliquement vers l'arrière par rapport au plan du palais (Fig. 7A). De fait, la M1 est très fortement recourbée dans sa partie intra-alvéolaire et le fragment MNHN AYO 188 correspondant à la M1 consiste en un élément de fût fortement arqué. Le fond de l'alvéole est situé au niveau de la partie supérieure du foramen infra-orbitaire, en avant de celui-ci, au voisinage immédiat de la M2. L'alvéole décrit ensuite un arc de cercle vers l'avant, s'écartant ainsi de la M2, dont le fût serait plus droit, avant de s'en rapprocher vers l'orifice alvéolaire. Cette forme de la M1 (Fig. 7A), semblable à celle de la C-, est différente de celle observée sur l'avant-crâne de l'holotype (GB 078; Fig. 5A, B) et chez Glossotherium, où seule la $\mathrm{C}$ - possède une telle courbure et dont la $\mathrm{M} 1$, très peu arquée, possède une forme plus voisine de celle de la M2 à laquelle elle est quasiment parallèle et dont elle n'est séparée que par une cloison verticale de moyenne épaisseur. Sur le spécimen MNHN AYO 188, la face postérieure est étroite, peu aplatie et il n'y a pas de face antérieure car, à ce niveau, le fût est très arrondi. Les faces labiale et linguale, les plus larges, sont marquées en leur centre d'un léger sillon longitudinal, mieux visible sur la face latérale. Chez l'holotype (GB 078), l'espace entre M1 et M2 est de même longueur que celui séparant $C$ - et M1 et semblablement réduit (Figs 5A, B ; 6A, B) alors que, chez le paratype (Fig. 7), il est plus développé que celui séparant C- et M1 (6 mm contre 2,6 mm).

Chez l'holotype (Figs 5A, B; 6A, B), l'alvéole de la $\mathrm{M} 2$ est réniforme et relativement arrondie. Chez le paratype (MNHN-BOL V 3321; Fig. 7A), la M2 possède plus la forme d'un triangle rectangle d'angle droit en position antérolinguale et son angle antérolabial est très arrondi. La face antérieure est rectiligne à légèrement concave comme chez Glossotherium, Paramylodon ou Glossotheridium et contrairement à ce que l'on peut observer chez Mylodonopsis et Pseudoprepotherium. La face postérolabiale de la M2 présente le même relief en plus marqué. La face postérolabiale semble proportionnellement plus longue que chez Glossotherium et Glossotheridium, ce qui confère à la partie postéromédiale de cette dent un développement plus important. La face linguale est la moins large et montre en son milieu un sillon très marqué, déterminant de part et d'autre deux petits lobes linguaux, le lobe postérieur étant un peu plus développé que l'antérieur (Fig. 5A, B). Sur le spécimen MNHN AYO 188, la table d'usure a sa normale dirigée ventralement et labialement. Comme chez Glossotherium, la forme de sa section est plus triangulaire que trapézoïdale. L'angle labial est arrondi chez Simomylodon n. gen., Glossotheridium, Paramylodon mais également chez Pleurolestodon et Mylodonopsis alors qu' il est aplati chez Glossotherium. La M3 du paratype est également de forme plus triangulaire que chez Glossotherium et présente un développement plus important de la partie postérolinguale (Fig. 7A). Cependant, l'angle antérolingual de la M3 est obtus alors qu'il est presque droit sur la M2, ce qui donne à la M3 une section d'aspect plus étiré labiolingualement que pour la M2. Sur le spécimen MNHN AYO 188, la face postérolabiale de la M3 porte un sillon bien marqué qui donne à la dent un aspect plus bilobé que chez Glossotherium et Glossotheridium (Fig. 7). L'alvéole de l'holotype présente également cette forme (Fig. 5A, B). Chez le paratype, le sillon postérolabial de la M3 ne semble pas aussi marqué, d'où une section plus triangulaire que bilobée (Fig. 7). Chez l'holotype, la paroi antérieure de l'alvéole de la M3 est orientée suivant une direction antérolinguale à postérolabiale, comme chez Glossotherium robustum, ce qui lui confère un contour trapézoïdal (Fig. 5A, B). Par contre chez le paratype, elle est orientée suivant un plan antérolabial à postérolingual, comme chez Glossotheridium chapadmalense, d'où un contour plus triangulaire.

La M4 est bilobée et possède un lobe antérieur d'une taille comparable à celui de la C- de l'holotype de Simomylodon n. gen. (Fig. 5A, B). L'isthme le séparant du lobe postérieur n'est qu'à peine plus étroit que ce dernier. Le lobe antérieur est de section elliptique de grand axe labiolingual sur les deux spé- 
cimens de Simomylodon n. gen. Le lobe postérieur est arrondi et correspondrait à une excroissance issue du quart labial de la moitié linguale du premier lobe. Corrélativement, le sillon labial est plus ample que le sillon lingual. Le lobe postérieur est nettement plus petit que l'antérieur comme chez Pseudoprepotherium contrairement à ce que l'on observe chez Glossotherium, Paramylodon et probablement Mylodonopsis (qui n'a conservé que l'alvéole dentaire de la M4) où ils sont d'égale importance. Ce dernier caractère distingue également Simomylodon n. gen. de Pleurolestodon dalenzae n. sp. et surtout de $P$. acutidens dont Kraglievich (1921) décrit un lobule postérieur plus allongé et plus étroit que chez tout autre Mylodontinae.

\section{Crâne (Figs 5-7; Tableau 2)}

Les éléments crâniens les plus complets dont nous disposons sont l'avant-crâne de l'holotype (GB 078) et le palais du paratype (MNHN-BOL V 3321). L'avant-crâne GB 078 (Figs 5, 6) est la pièce la mieux conservée même si toutes les dents font défaut. Elle correspond à la région du museau, antérieure à un plan transversal passant en arrière de l'alvéole de la M4. Le paratype MNHN-BOL V 3321 (Fig. 7) est constitué des maxillaires droit et gauche d'un individu, avec les bases antérieures des arcades zygomatiques et les parties intra-alvéolaires des séries M2-M4 droite et M1-M3 gauche.

\section{Prémaxillaire (Figs 5-7)}

En vue ventrale, l'holotype présente des prémaxillaires en forme "d'ancre de marine" (Fig. 5A, B). Leurs processus médiaux s'accolent dans un plan sagittal et s'arrêtent au niveau d'une ligne passant par le centre des alvéoles des C- alors que leurs processus latéraux s'arrêtent avant d'atteindre ces mêmes alvéoles (Fig. 3A, B). Le contour antérieur du palais situé entre les C- présente l'aspect d'un arc surbaissé, aplati au niveau de la région sagittale. Ce trait différencie Simomylodon uccasamamensis n. gen., n. sp. de Glossotherium wegneri, G. robustum et Pleurolestodon dalenzae n. sp. La forme quaternaire du Brésil Mylodonopsis ibseni présente des prémaxillaires très différents de ceux de Simomylodon n. gen., extrêmement massifs et comparables à ceux de Mylodon darwini. Chez Glossotherium et Pleurolestodon mais surtout chez Mylodonopsis et Mylodon, les prémaxillaires se projettent vers l'avant dans la région sagittale, sans doute pour supporter des éléments cartilagineux inter-nasaux. En relation avec ce caractère, en vue ventrale, le contour antérieur du prémaxillaire entre les $\mathrm{C}^{-}$et la suture inter-prémaxillaire est convexe alors qu'il est sigmoïde chez Glossotherium et Pleurolestodon. Chez l'holotype de Glossotheridium chapadmalense, les prémaxillaires n'ont pas été conservés mais, par l'extension, en avant du niveau des $\mathrm{C}^{-}$, des processus antérieurs des lames palatines des maxillaires, il est probable que cet animal présentait une morphologie prémaxillo-maxillaire semblable à celle de Glossotherium et Pleurolestodon.

Chez Simomylodon n. gen., le bord antérieur des prémaxillaires s'abaisse vers l'avant et constitue ainsi un bord incisif supérieur opposé à celui de la région symphysaire de la mandibule (Figs 6A, B; 7B).

\section{Maxillaire (Figs 5-7)}

En vue ventrale, on discerne un élargissement des maxillaires vers l'avant comme chez Glossotherium robustum, G. wegneri, Mylodonopsis ibseni, Urumacotherium campbelli et Glossotheridium chapadmalense (Kraglievich 1921, 1925; Hoffstetter 1952; Frailey 1986; Cartelle 1991), caractère corrélatif avec la divergence vers l'avant des séries dentaires, bien marquée chez ces espèces (Figs 5A, B; 7A). Cet élargissement est mieux marqué que chez Mylodonopsis ibseni, Pseudoprepotherium confusum, Paramylodon harlani et surtout Mylodon darwini (Stock 1925; Kraglievich 1934; Hirschfeld 1985; Cartelle 1991). Alors que chez Pleurolestodon acutidens la distance séparant les faces internes des C- est inférieure au double de celle séparant les faces internes des M4, le rapport est supérieur à 2 chez Simomylodon n. gen. comme chez Glossotherium.

Le processus du maxillaire s'engageant entre les processus latéral et médial du prémaxillaire correspondant offre un contour plus arrondi, moins pointu médialement que chez Glossotherium robustum, G. wegneri ou Paramylodon harlani (Stock 1925). Ce caractère est sans doute en relation avec l'aspect non proéminent du bord incisif au niveau de la suture inter-prémaxillaire (Figs 5A, B ; 7A). 


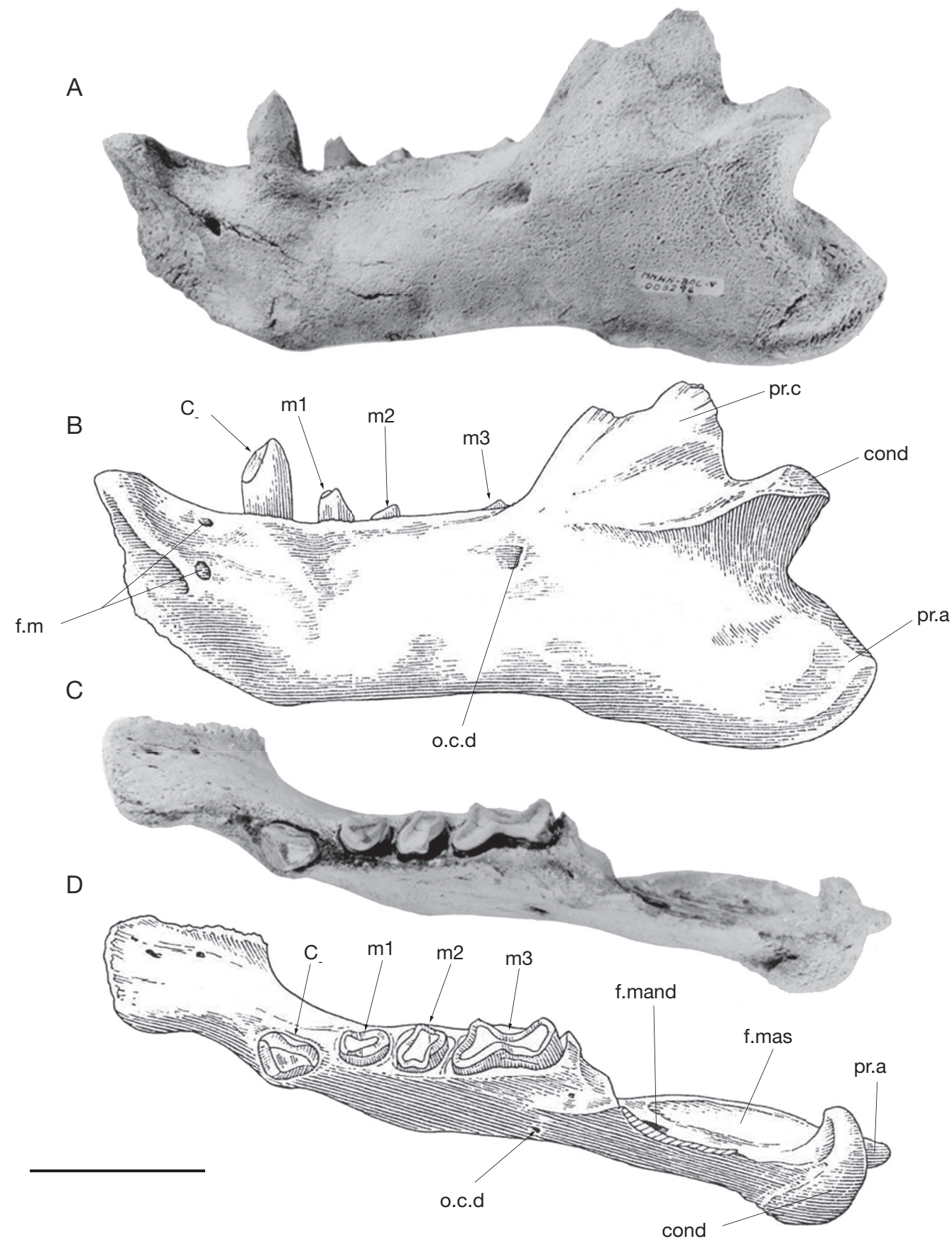

FIG. 8. - Hémimandibule gauche (MNHN-BOL V 3296) attribuée à Simomylodon uccasamamensis n. gen., n. sp.: A, B, vue latérale gauche (antérieure vers la gauche); C, D, vue occlusale (antérieure vers la gauche). Abréviations: C., dent caniniforme inférieure; cond, condyle; f.m, foramens mentonniers; f.mand, foramen mandibulaire (ouverture postéromédiale); f.mas, fosse massétérine; o.c.d, orifice postéro-externe du canal dentaire; m1-m3, dents molariformes inférieures; pr.a, processus angulaire; pr.c, processus coronoïde. Échelle: $5 \mathrm{~cm}$. 
Chez Lestodon armatus, Glossotherium robustum, $G$. wegneri et Glossotheridium chapadmalense et contrairement à ce qui est observé chez Pseudoprepotherium confusum et Mylodonopsis ibseni, les bords latéraux des maxillaires ne s'étendent pas en avant de la C-.

Le palais présente de nombreux foramens dont les deux plus importants se trouvent de part et d'autre du plan sagittal à la hauteur de la cloison interalvéolaire C--M1 (Figs 5A, B; 7A).

La base antérieure de l'arcade zygomatique est située sur la face latérale du maxillaire à un niveau s'étendant entre la moitié antérieure de la M2 et l'extrémité postérieure de la M3 (Figs 5A, B; 6A, B ; 7A), comme chez Glossotherium. Elle est percée du foramen infra-orbitaire dans sa partie inférieure dont le plancher est de ce fait nettement plus mince que le toit (Figs 5A, B; 6A, B). Chez Glossotherium wegneri, le plancher de ce foramen peut même disparaître (Hoffstetter 1952).

Une différence, aisément observable entre Simomylodon n. gen. et Pleurolestodon d'une part, et Glossotherium et Glossotheridium d'autre part, est la forme du museau en coupe transversale en avant de l'attache antérieure de l'arcade zygomatique. Alors que chez ces derniers genres, la largeur maximale se situe au niveau d'un plan frontal passant parallèlement à celui du palais, nettement au-dessus de celui-ci et au niveau de la racine antérieure de l'arcade zygomatique, chez Simomylodon n. gen. et Pleurolestodon la largeur maximale du museau se trouve au niveau des bords alvéolaires labiaux (Figs 3; 5A, B). En vue ventrale, ceux-ci constituent le contour externe du crâne, alors que, chez les espèces attribuées à Glossotherium, ce contour correspond au niveau de la paroi latérale des fosses nasales où la largeur transversale est maximale.

\section{Nasaux (Figs 5-7)}

L'un des caractères morphologiques les plus remarquables de Simomylodon n. gen. par rapport aux espèces du genre Glossotherium est l'absence, à l'extrémité antérieure de la suture internasale, de prolongements libres des nasaux en avant des limites latérales de l'orifice antérieur des fosses nasales. Il semblerait même qu' existât, au niveau de l'extrémité antérieure de cette suture, une petite échancrure.
En vue latérale, l'extrémité antérodorsale des nasaux est en retrait, n'atteignant pas le niveau de l'extrémité antérieure des prémaxillaires (Fig. 6A, B), contrairement à ce que l'on observe chez $G$. wegneri (Hoffstetter 1952).

\section{Dents inférieures (Figs 8; 9; Tableau 2)}

La $C_{-}$est conservée partiellement sur les spécimens MNHN AYO 165, AYO 185 et AYO 186 (Fig. 9A), MNHN-BOL V 3298 et entièrement sur le spécimen MNHN-BOL V 3296 (Fig. 8). L'emplacement de l'alvéole est marqué sur la face latérale de la branche horizontale par une extumescence plus faible que chez Glossotherium robustum. Elle est nettement caniniforme et proportionnellement plus grande (par rapport aux autres dents) que chez Glossotheridium chapadmalense, Pleurolestodon acutidens, Pseudoprepotherium confusum et Mylodon darwini et quelque peu semblable à ce que l'on peut observer chez Glossotherium et Kiyumylodon. Comme chez Glossotherium, Glossotheridium, Kiyumylodon et Pseudoprepotherium, sa couronne est usée en double biseau (Fig. 8A, B). Le biseau antérieur, le plus large, présente sa normale dirigée latéralement par rapport au plan sagittal, et la droite passant par l'arête sommitale est orientée antérolinguallement à postérolabiallement. Sa section est régulièrement réniforme. En effet la face linguale est soit plane (MNHN-BOL $\mathrm{V}$ 3298) soit très légèrement concave (MNHNBOL V 3296; Fig. 8C, D) et le reste du contour est presque semi-circulaire. En vue occlusale, la $\mathrm{C}_{\text {_ }}$ est nettement triangulaire en section contrairement à celle des autres Mylodontinae et notamment de Kiyumylodon (Rinderknecht et al. 2007).

Sur le spécimen MNHN-BOL V 3296, la m1 possède une surface d'usure principale dirigée postérolingualement et de profil central concave (Fig. 8A, B). La vasodentine centrale détermine en effet une dépression par rapport à la zone périphérique du fût constituée d'ostéodentine plus dure (Fig. 8C, D). Celle-ci, sur le bord antérieur et antérolabial, est usée en un biseau de petite taille qui n'affecte pas la vasodentine. La courbe passant par l'arête sommitale de ce double biseau est fortement concave et se confond, au milieu de la dent, avec la limite entre la vasodentine et 

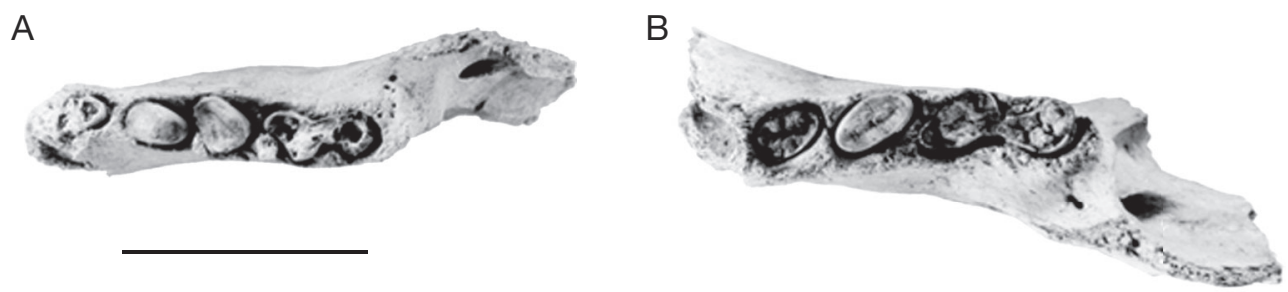

FIG. 9. - Restes mandibulaires attribués à Simomylodon uccasamamensis n. gen., n. sp. : A, branche horizontale d'une hémimandibule droite (MNHN AYO 165, spécimen juvénile), vue occlusale (antérieure vers la gauche); B, fragment d'une hémimandibule gauche (MNHN VIZ 1), vue occlusale (antérieure vers la gauche). Échelle: $5 \mathrm{~cm}$.

l'ostéodentine. Sa direction est semblable à celle de la C. La section de la $\mathrm{m} 1$ est réniforme. La paroi médiale du fût est légèrement concave et la région antérieure arrondie est plus volumineuse que la région postérieure (Fig. 8C, D). De plus, le contour antérieur est nettement plus arrondi que chez Glossotherium, Pleurolestodon, Paramylodon et Glossotheridium. Sur le spécimen MNHN AYO 165 (Fig. 9A), qui correspond à la partie d'une hémimandibule droite ayant appartenu probablement à un individu juvénile, la section de la $\mathrm{m} 1$ est réniforme et semblable à celle de la m1 du spécimen MNHN-BOL V 3296 (Fig. 8C, D) mais le sillon médial y est peu marqué. Le fût est légèrement incliné vers l'avant et s'élève plus haut que celui de la m2 (Fig. 8A, B). La surface occlusale est usée en double biseau. Le biseau antérieur a sa normale dirigée vers l'avant et légèrement en direction de la face labiale, alors que la normale au plan du biseau postérieur est dirigée vers l'arrière et un peu médialement. La surface du biseau antérieur est, en projection sur la section, environ deux fois plus grande que celle du biseau postérolingual.

La m2 du spécimen MNHN-BOL V 3296 est de section grossièrement rectangulaire, contrairement à celle de la majorité des autres Mylodontinae (Fig. 8C, D). Ses faces latérale, médiale et postérieure présentent des sillons peu profonds alors que la face antérieure est parfaitement plane. Sur le spécimen MNHN-BOL V 3298, les faces latérale et médiale ne sont marquées d'aucun sillon. Les faces antérieure et postérieure sont les plus larges et le grand axe de la section est dirigé antérolabiallement à postérolinguallement. Le fût est moins comprimé antéropostérieurement que chez Glossotherium, Paramylodon et Glossotheridium. La surface occlusale est usée suivant deux biseaux dont les normales se rapprochent plus de l'axe vertical de la dent que sur les $C_{-}-m 1$. En effet, comme le biseau antérieur est séparé du postérieur par une ligne sommitale joignant le tiers labial de la face postérieure au tiers antérieur de la face linguale, le biseau postérieur présente un plus faible développement que l'antérieur. Sur la couronne, les phénomènes de relief dus à l'usure différentielle de l'ostéodentine et de la vasodentine sont similaires à ceux observés sur la m1. Sur le spécimen MNHN AYO 165, la face postérieure de la m2 est plus convexe, il n'existe pas d'angle postérolabial et la région latérale de la dent est arrondie. L'ostéodentine pariétale s'élève depuis le bord labial de la face linguale, d'une part jusqu'au centre de la face linguale et, d'autre part, jusqu'au milieu de la face postérieure, constituant ainsi deux pointes, la première (linguale) plus haute que la seconde (postérieure). La section elliptique de la m2 du spécimen MNHN VIZ 1 (Fig. 9B) représente une variation individuelle, absente sur tous les autres spécimens.

La m3 est bilobée comme chez tous les Mylodontidae. La section du lobe antérieur est réniforme; son grand axe est antérolabial à postérolingual mais l'étirement du lobe suivant cet axe est moindre que chez Glossotherium, Kiyumylodon, Paramylodon et Pleurolestodon. La face antérieure est marquée d'un sillon délimitant une partie labiale et une partie linguale, la première plus développée, la seconde plus arrondie qui provoque une face antérolinguale concave et non convexe comme chez Kiyumylodon. Le lobe postérieur moins déve- 
loppé que l'antérieur, est relié à celui-ci par un isthme, dont la paroi linguale est régulièrement arrondie. La paroi labiale de l'isthme et du lobe postérieur est rectiligne avec un léger ressaut vers la partie postérieure de l'isthme. Le grand axe de ce lobe postérieur est orienté labiolingualement avec une légère composante antéropostérieure, comme chez Glossotherium et Kiyumylodon, alors que, chez Glossotheridium, il n'est orienté que labiolingualement.

\section{Dentaire (Figs 8; 9)}

Le dentaire le mieux conservé est celui du spécimen MNHN-BOL V 3296 (Fig. 8), hélas dépourvu de l'extrémité du processus coronoïde.

\section{Branche ascendante (Fig. 8)}

La base du processus coronoïde ne montre aucune trace de la crête observée sur la paroi latérale de Pleurolestodon acutidens (Kraglievich 1921). Sur la face médiale, le foramen mandibulaire se situe à la base du processus coronoïde, en arrière de la série dentaire, au-dessus et en avant de la fosse d'insertion pour le ptérygoïdien latéral (Fig. 8C, D). Sur la face latérale, en dessous du bord antérieur du processus coronoïde, au niveau du lobe postérieur de la dernière molaire, s'ouvre un petit foramen du canal dentaire (Hoffstetter 1952, pour Glossotherium wegneri).

Le condyle est réniforme en vue supérieure et très saillant médialement. Latéralement, il s'amincit en une courte crête descendant obliquement vers l'avant sur la face externe de la branche ascendante (Fig. 8A, B), comme chez Glossotherium wegneri mais contrairement à ce que l'on discerne par exemple chez Pleurolestodon acutidens (Kraglievich 1921; Hoffstetter 1952).

L'incisure située entre le condyle et l'apophyse angulaire présente, en vue latérale et en vue postérieure, un profil particulier constitué de deux crêtes superposées dans un plan vertical, s'abaissant d'avant en arrière et dessinant, en vue postérieure, une figure en forme de " 3 " aux concavités très largement ouvertes médialement.

Le processus angulaire bien développé se projette fortement vers l'arrière, légèrement vers le bas et son extrémité est très postérieure par rapport au condyle (Fig. 8A, D).

\section{Branche horizontale (Fig. 8)}

La symphyse est haute et son bord postéroventral atteint à peine le niveau du bord alvéolaire antérieur de la première dent. En vue latérale, son profil présente l'aspect d'une courbe sigmoïde convexe entre les foramens mentonniers et concave entre ce niveau et le bord symphysaire (Fig. 8A, B). Sa partie antérodorsale, communément appelée "bec» chez les tardigrades, est élargie et aplatie comme chez Kiyumylodon, Mylodon et Glossotherium, ce qui la différencie de celle de Pleurolestodon acutidens chez lequel la mandibule ne ressemble pas à une "pelle» mais plutôt au «bec d'une cruche» (voir les spécimens MACN Pv 2953 dans Rovereto [1914] et FMNH P14495) mais également de Lestodon chez qui le bec en forme de "pelle» est développé à l'extrême. La symphyse est fortement saillante pratiquement à la manière d'une crête (Kraglievich 1921) un peu comme chez Pleurolestodon. Le bord symphysaire en vue antérieure est presque horizontal, bien que sa face linguale soit légèrement concave à cause de l'épaississement des bord latéraux de la pelle symphysaire, épaississement situé dans le prolongement du pilier séparant de chaque côté les deux foramens mentonniers principaux. En vue occlusale, le bord symphysaire est relié à la première alvéole dentaire par une zone plus étroite, comme chez la majorité des Mylodontinae (Fig. 8C, D). La largeur de la région symphysaire est plus proche de celle observée chez Glossotheridium, Kiyumylodon et Mylodon que chez Glossotherium robustum. Le nombre des foramens mentonniers est de deux par hémimandibule sur toutes les pièces, sauf sur le spécimen MNHN-BOL V 3298 où il y en a six. À cette exception près, ce dernier spécimen présente les mêmes caractéristiques que les autres.

La région pré-alvéolaire est plus courte que chez Mylodon darwini, Pseudoprepotherium confusum, Glossotheridium chapadmalense et, dans une moindre mesure, que chez Glossotherium robustum et Paramylodon harlani (Lydekker 1894; Stock 1925). Ce caractère est sans doute lié à la faible longueur des prémaxillaires chez Simomylodon n. gen. En vue latérale, le bord inférieur de la mandibule présente un renflement au niveau de la C. (Fig. 8A, B). En avant de ce point, il se dirige dorsalement vers l'extrémité postérieure de la symphyse. Vers l'arrière, 


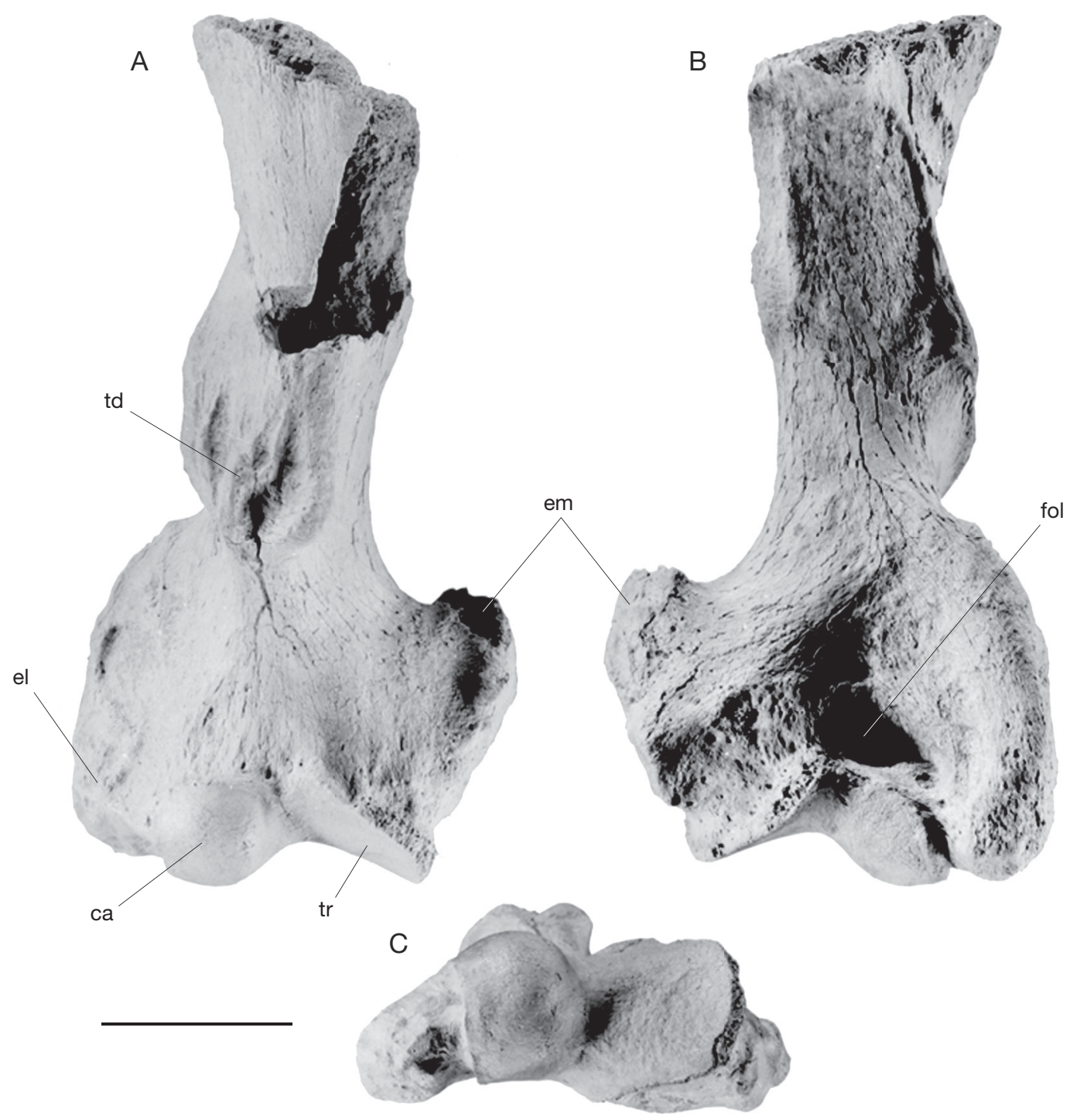

FIG. 10. - Humérus droit (MNHN AYO 110) attribué à Simomylodon uccasamamensis n. gen., n. sp.: A, vue antérieure (proximale vers le haut); B, vue postérieure (proximale vers le haut); C, vue distale (antérieure vers le haut). Abréviations: ca, capitulum; el, épicondyle latéral; em, épicondyle médial; fol, fosse olécrânienne; td, tubérosité deltoïdienne; tr, trochlée. Échelle: $5 \mathrm{~cm}$.

il est rectiligne jusqu'à l'apophyse angulaire dont le bord inférieur s'infléchit ventralement.

\section{Membre antérieur (Figs 10-15; Tableau 3)}

Humérus (Fig. 10). Les nombreux spécimens récoltés à Ayo Ayo-Viscachani permettent une bonne connaissance de l'humérus de Simomylodon n. gen.
La tubérosité deltoïdienne est plus proéminente que chez Lestodon armatus, Paramylodon harlani et Glossotherium robustum (Fig. 10A) et semblable à ce que l'on observe chez Pseudoprepotherium confusum (Hirschfeld 1985). En effet son bord médial est mieux détaché de la diaphyse par rapport au plateau huméral distal et son extrémité distale est mieux délimitée. Par contre 


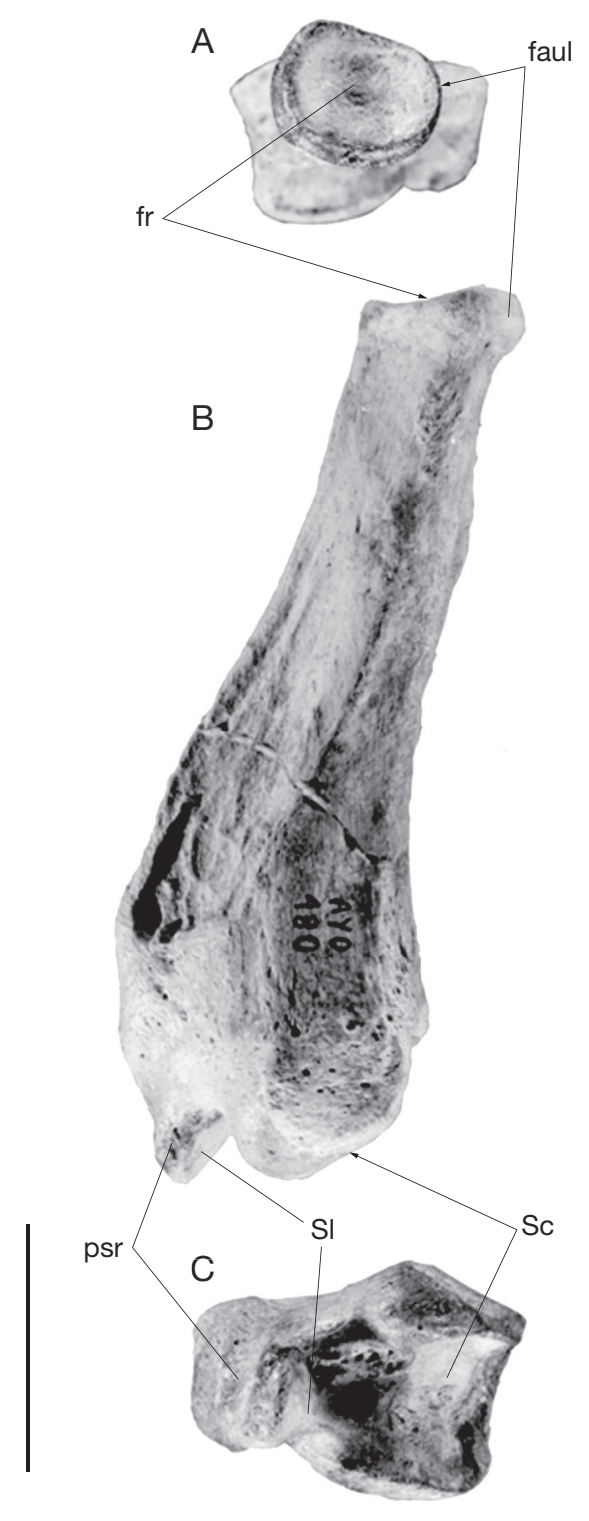

FIG. 11. - Radius (MNHN AYO 110) gauche attribué à Simomylodon uccasamamensis n. gen., n. sp.: A, vue proximale (latérale vers le haut); B, vue antérieure (proximale vers le haut); C, vue distale (antérieure vers le haut). Abréviations: faul, facette articulaire pour l'ulna; fr, fovea du radius; psr, processus styloïde radial; Sc, scaphoïde; SI, semilunaire. Échelle: $5 \mathrm{~cm}$.

cette tubérosité est proportionnellement plus étroite que chez ces deux derniers genres.

La crête épicondylienne (latérale) est une lame proportionnellement moins développée en largeur que chez Glossotherium. En vue antérieure ainsi que de profil, sa partie proximale est nettement plus arrondie que chez Glossotherium, Paramylodon harlani et Lestodon armatus. Chez ces dernières, le contour de cette crête forme un angle dans sa partie proximolatérale. L'ectépicondyle est plus développé que chez Pseudoprepotherium confusum et Paramylodon harlani. L'épitrochlée (épicondyle médial) forme un processus ascendant d'un développement semblable à celui observé chez Glossotherium et Paramylodon mais plus bas et mieux séparé de la diaphyse que chez Lestodon. Un tel processus n'existe pas chez Pseudoprepotherium confusum.

En vue postérieure (Fig. 10B), la fosse olécrânienne est plus distincte que chez les grands Glossotherium pléistocènes. L'extension postérieure de celle-ci est moindre que chez Glossotherium alors que celles du condyle et de la gorge sont plus importantes. Les surfaces articulaires distales ressemblent beaucoup plus à celles de Glossotherium qu'à celles de Pseudoprepotherium et Paramylodon et la gorge de la trochlée est bien marquée (Fig. 10C). La relative réduction de l'extension latérale de la crête épicondylienne est bien visible en vue postérieure.

Radius (Fig. 11). La forme générale est comparable à celle du radius de Glossotherium robustum. Il est beaucoup plus court et massif chez Paramylodon harlani (Stock 1925) alors que chez Pseudoprepotherium, l'extrémité proximale est plus développée transversalement et l'os est dans son ensemble un peu plus court et corpulent (Hirschfeld 1985). La différence majeure entre Simomylodon n. gen. et Glossotherium réside dans la morphologie du bord médial. Dans les deux tiers distaux, le bord médial forme une crête de profil convexe jusqu'au processus styloïde radial alors que chez Glossotherium robustum le profil est relativement rectiligne jusqu'au tubercule surmontant ce processus. En vue proximale (Fig. 11A), la tête est moins bien séparée de la diaphyse car le col est moins marqué que chez $G$. robustum. Chez cette espèce, la tête est d'un diamètre notablement plus grand que l'extrémité distale de la diaphyse (Tableau 3) tandis que chez Simomylodon n. gen. ce diamètre est tout-à-fait comparable. La seule légère saillie proximale marquée à ce niveau est la facette arti- 


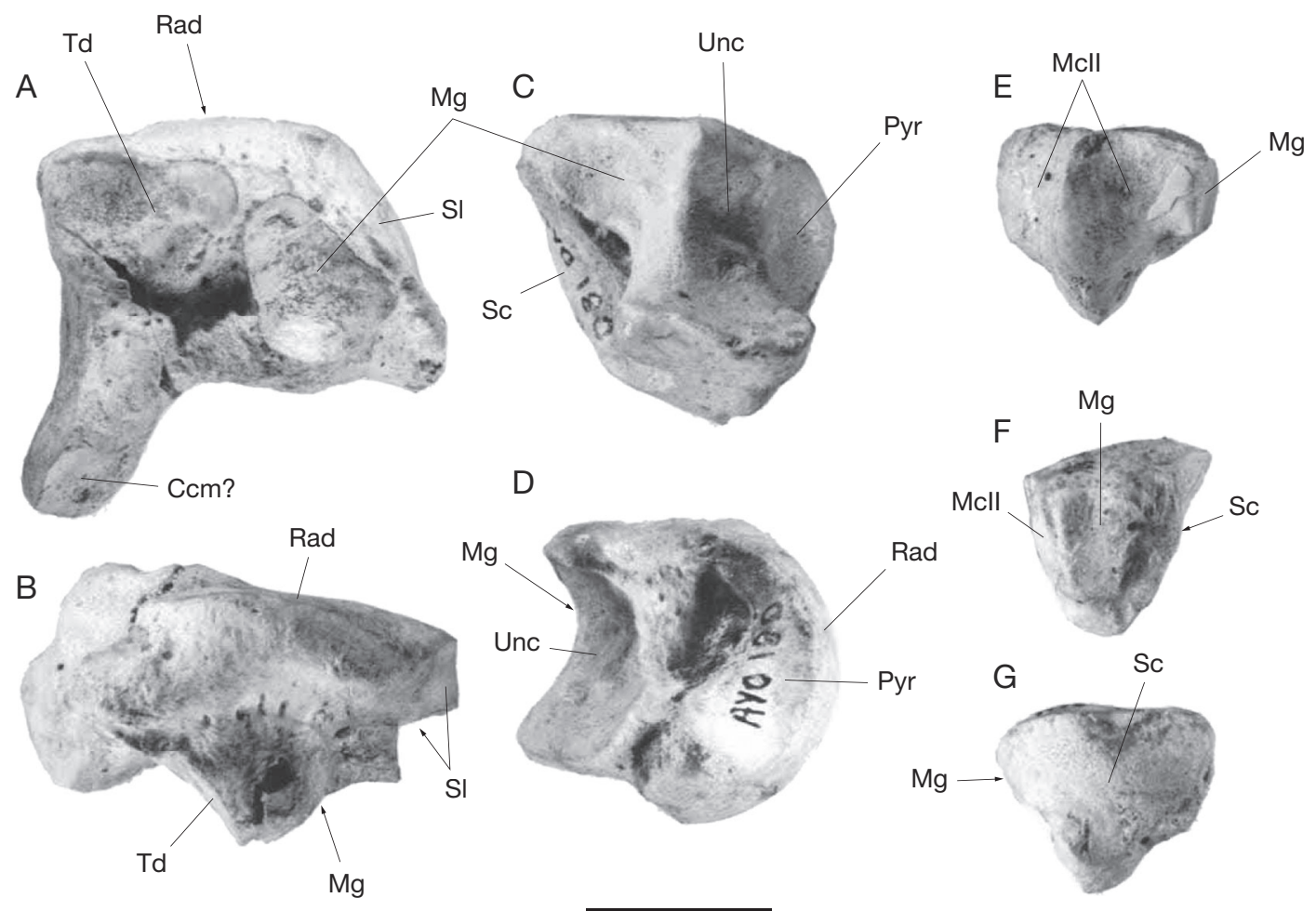

FIG. 12. - Carpiens de la main de Simomylodon uccasamamensis n. gen., n.sp.: A, B, scaphoïde gauche (MNHN AYO 180) en vues médiodistale (dorsale vers le haut, médiale vers la gauche) et dorsale (proximale vers le haut, médiale vers la gauche) respectivement; C, D, semilunaire gauche (MNHN AYO 180) en vues distale (dorsale vers le haut, médiale vers la gauche) et latérale (dorsale vers le haut, distale vers la gauche) respectivement; E-G, trapézoïde gauche (MNHN AYO 180) en vues distale (dorsale vers le haut, médiale vers la gauche), latérale (dorsale vers le haut, distale vers la gauche) et proximale (dorsale vers le haut, médiale vers la gauche) respectivement. Abréviations: Ccm, complexe carpo-métacarpe; Mcll, métacarpe II; Mg, magnum; Pyr, pyramidal; Rad, radius; Sc, scaphoïde; SI, semilunaire; Td, trapézoïde; Unc, unciforme. Échelle: $2 \mathrm{~cm}$.

culaire pour l'ulna, elle-même proportionnellement moins importante que chez Glossotherium, surtout en vue proximale, par rapport à la surface d'articulation avec l'humérus.

Le processus antéro-externe de l'extrémité distale est un peu plus développé distalement que chez $G$. robustum. La facette d'articulation avec le scaphoïde est légèrement plus concave en vue dorsale (Fig. 11C), plus étroite dorsoventralement et son bord palmaire est plus rectiligne. Enfin le sillon séparant ce processus du processus styloïde est proportionnellement plus profond.

Scaphoïde (Fig. 12A, B). La facette d'articulation avec le trapézoïde est étirée transversalement et non subcirculaire comme chez Glossotherium robustum. Cependant, cette facette et sa voisine en relation avec le magnum, très proches l'une de l'autre, ne sont pas jointives comme chez ce dernier. La facette articulant le scaphö̈de avec le semi-lunaire semble proportionnellement plus étroite et plus allongée en direction palmaire où elle se termine en formant une protubérance à peine ébauchée chez $G$. robustum. Le scaphoïde est plus développé transversalement et le processus médial plus gracile chez Pseudoprepotherium que chez Simomylodon $\mathrm{n}$. gen. À l'extrémité du processus médial est présente une petite surface articulaire ovale probablement pour le doigt interne ou plus probablement un complexe carpo-métacarpe. 

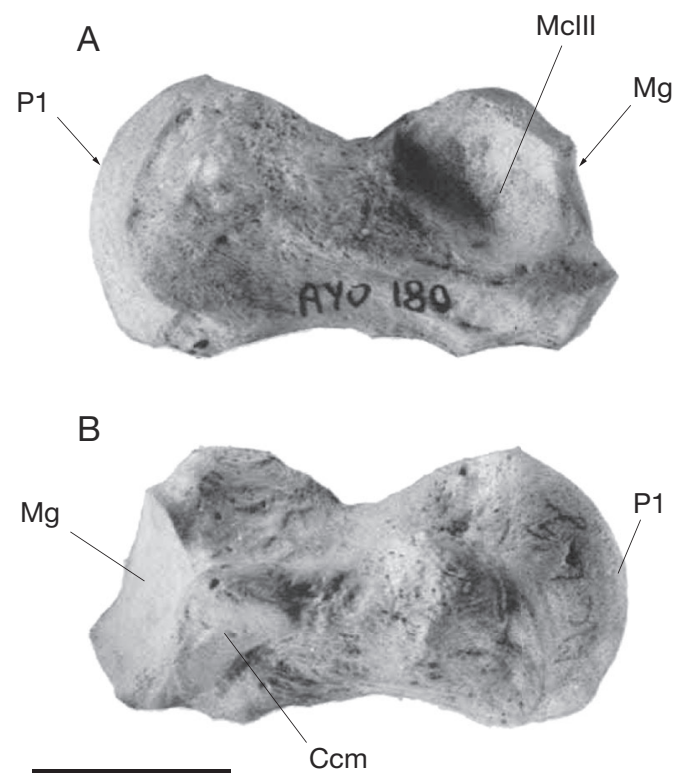

FIG. 13. - Métacarpien II gauche (MNHN AYO 180) de Simomylodon uccasamamensis n. gen. n.sp.: A, vue latérale (dorsale vers le haut, distale vers la gauche); $\mathbf{B}$, vue médiale (dorsale vers le haut, proximale vers la gauche). Abréviations: $\mathbf{C c m}$, complexe carpo-métacarpe; McIII, métacarpe III; Mg, magnum; P1, première phalange. Échelle: $2 \mathrm{~cm}$.

Semilunaire (Fig. 12C, D). La surface articulaire pour le pyramidal est proportionnellement plus grande et plus concave que chez Glossotherium robustum. La partie palmaire de l'articulation avec l'unciforme est plus élargie que la partie dorsale, contrairement à ce que l'on observe chez $G$. robustum. L'angle que font entre eux les plans des facettes pour le magnum et pour l'unciforme est très légèrement obtus alors qu'il est presque plat chez Pseudoprepotherium confusum et aigu chez $G$. robustum.

Les facettes pour le scaphoïde et le magnum sont séparées alors que, chez $G$. robustum, elles fusionnent au niveau de la partie la plus dorsale de la première facette. Les plans de ces deux facettes forment un angle obtus alors qu'il est plat chez Pseudoprepotherium. La fosse séparant l'articulation avec le magnum de celle avec le scaphoïde est plus large et plus profonde que chez $G$. robustum. La facette d'articulation avec le scaphoïde est plus arquée, caractère corrélatif avec la plus forte convexité de la facette pour le radius.
Trapézoïde (Fig. 12E-G). Le trapézoïde possède une surface d'articulation qui, faisant suite à celle portée par le métacarpien II pour le métacarpien III, occupe la même position que la facette articulant le trapézoïde avec le magnum de $G$. robustum. Cette surface est plane alors que, chez ce dernier, elle est concave.

La surface postérieure articulant le trapézoïde avec le scaphoïde est nettement plus large que chez G. robustum et s'étend davantage en direction médiolatérale que dorsoventrale, ce qui fait que ce carpien est plus large que haut, contrairement à ce que l'on remarque chez ce dernier.

Pyramidal. Le spécimen MNHN AYO 109 est plus comprimé médiolatéralement que ce que l'on observe chez Glossotherium robustum (voir Tableau 3). Contrairement à ce qui est observé chez ce dernier, la hauteur dorsopalmaire est plus grande que la largeur médiolatérale. Ce caractère retentit sur la forme de la facette pour l'ulna, beaucoup moins large que chez $G$. robustum. La région palmaire de la facette pour l'unciforme est moins proéminente en direction distale et sa partie dorsomédiale est moins développée. Le bord dorsolatéral de cette facette est dépourvu d'expansion de la surface articulaire comme c'est le cas chez G. robustum.

Métacarpien II (Fig. 13). Le métacarpien II est proportionnellement plus long et plus fin que chez $G$. robustum et ressemblerait davantage, par sa forme générale et la compression médiolatérale qui l'affecte, à celui de Pseudoprepotherium confusum où il est encore plus comprimé, spécialement dans sa partie distale (voir Tableau 3). Comme chez celui-ci, le métacarpien II possède un corps séparant les extrémités distale et proximale, alors qu'elles sont «télescopées» chez $G$. robustum.

Les facettes pour le métacarpien III et le magnum sont bien séparées comme chez ce dernier, contrairement à ce que l'on observe chez Pseudoprepotherium. La facette d'articulation pour le métacarpien III est moins concave et bien plus petite que chez $G$. robustum. Chez Simomylodon n. gen., son bord distal est très éloigné de l'articulation avec la phalange.

En vue médiane (Fig. 13B), l'articulation avec le complexe carpo-métacarpe (ou le métacarpien I) est 

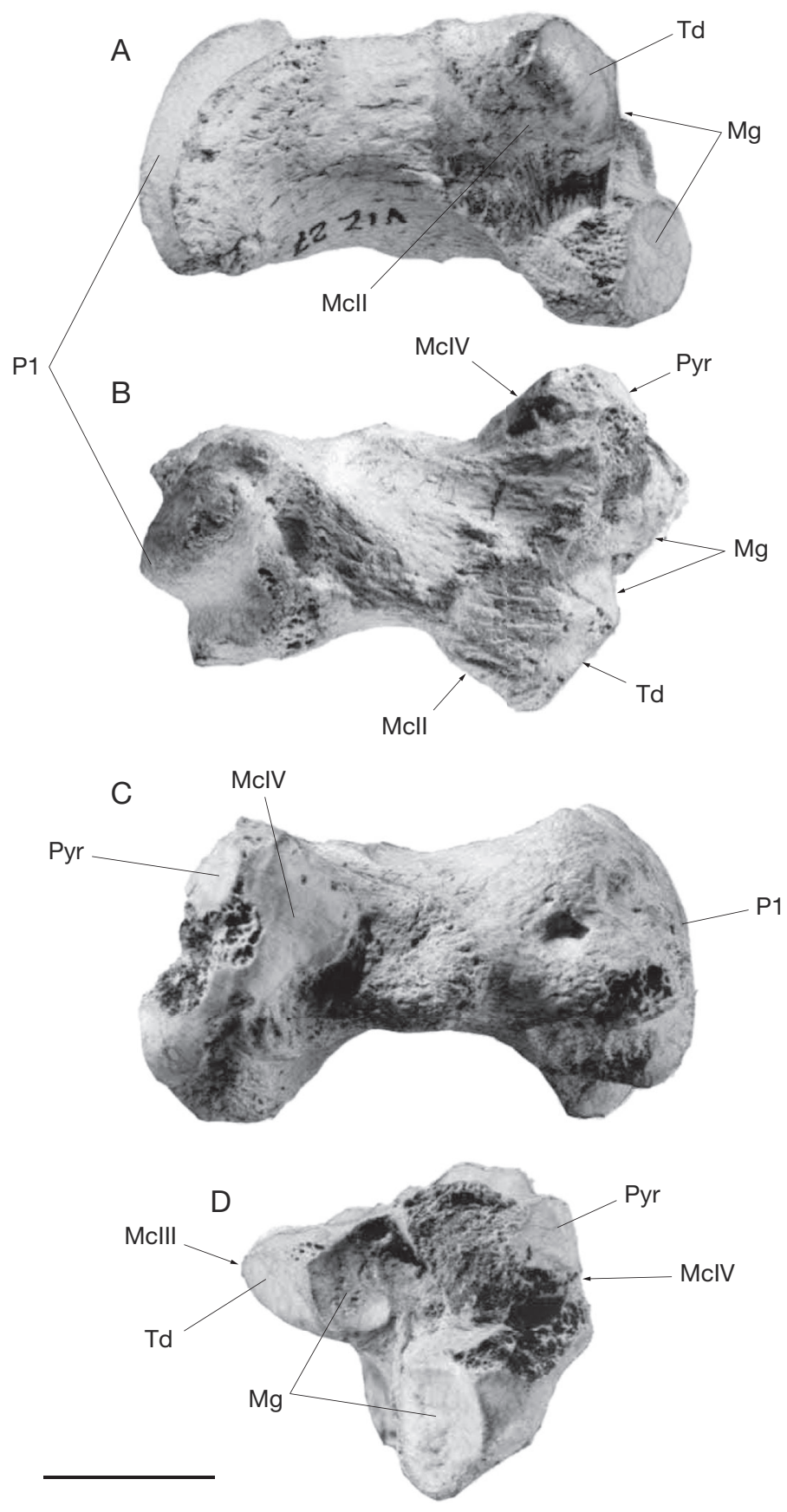

FIG. 14. - Métacarpien III droit (MNHN VIZ 27) attribué à Simomylodon uccasamamensis n. gen., n.sp.: A, vue médiale (dorsale vers le haut, distale vers la gauche); B, vue dorsale (latérale vers le haut, distale vers la gauche); C, vue latérale (dorsale vers le haut, proximale vers la gauche); D, vue proximale (dorsale vers le haut, médiale vers la gauche). Abréviations: McIl-IV, métacarpes II-IV; Mg, magnum; P1, première phalange; Pyr, pyramidal; Td, trapézoïde. Échelle: 2 cm. 


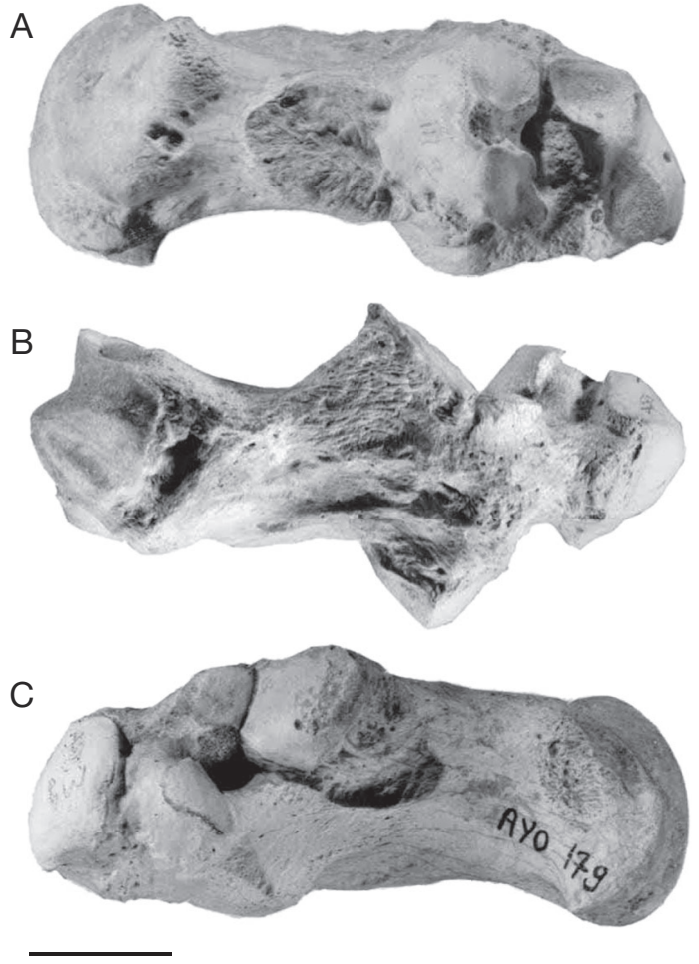

FIG. 15. - Métacarpien III et magnum gauche soudés (MNHN AYO 179) attribués à Simomylodon uccasamamensis n. gen., n. sp.: A, vue latérale (dorsale vers le haut, distale vers la gauche); $\mathbf{B}$, vue dorsale (médiale vers le haut, distale vers la gauche); C, vue médiale (dorsale vers le haut, proximale vers la gauche). Échelle: $2 \mathrm{~cm}$.

relativement petite, en position postérieure et plane à légèrement convexe, alors que chez $G$. robustum, où elle atteint le niveau de la partie dorsale de l'articulation avec la phalange, elle est très fortement concave.

Cet aspect relativement plat des facettes pour le complexe carpo-métacarpe (ou le métacarpien I) et le métacarpe III correspondrait à la condition plésiomorphe puisqu'il se retrouve chez Pseudoprepotherium, qui est considéré comme le Mylodontinae le plus primitif (Gaudin 2004). Il en est de même pour la faible extension postérodorsale de la carène de l'articulation avec la phalange. Chez Gl. robustum, le profil de cette carène est arrondi en vue latérale alors qu'il est plus rectiligne dans sa partie moyenne chez Pseudoprepotherium confusum (la variabilité individuelle chez cette espèce ne permet pas d'être trop catégorique sur ce point).

Métacarpien III et fusion métacarpien III-magnum (Figs 14; 15). Deux spécimens enregistrés dans les lots MNHN AYO 179 et MNHN AYO 180 correspondent à des métacarpiens III soudés aux magnums correspondants (Fig. 15). Les éléments MNHN VIZ 27 et MNHN VIZ 5 sont des métacarpiens III libres (Fig. 14).

Il existe au niveau de la soudure des deux « métacarpiens III-magnums» un large foramen qui traverse l'os dans sa largeur (Fig. 15). Au-dessus et en avant de ce foramen, côté médial, on découvre une facette d'articulation pour le métacarpien II ; en arrière et en dessous de ce foramen, existe une deuxième facette pour cet os (Fig. 15C). Enfin la facette articulant les métacarpiens II et III est moins convexe que chez Glossotherium robustum. La facette pour le métacarpien IV ne se prolonge pas vers l'avant sur la face latérale du corps du métacarpien III comme cela s'observe chez cette dernière espèce (Fig. 14).

Comme chez $G$. robustum, il existe une facette pour le trapézoïde située juste en arrière de la facette d'articulation du Mc III avec le métacarpien II, et au-dessus du foramen et de la facette magnum-Mc II. Cette facette magnum-trapézoïde est séparée de la facette métapodiale pour le métacarpien II par une très fine strie matérialisant la limite entre les deux éléments soudés.

Les facettes d'articulation du magnum avec le semi-lunaire et le scaphoïde s'étendent moins dorsoventralement que chez $G$. robustum. À l'encontre de ce que l'on peut observer chez celui-ci, les régions dorsales ne présentent aucune concavité.

Les Mc III isolés du site de Viscachani, sont identiques à la partie homologue des "Mc IIImagnum» provenant d'Ayo Ayo. Ils permettent la comparaison des facettes Mc III-magnum avec celles d'autres espèces, comparaison impossible à partir des éléments provenant d'Ayo Ayo.

Sur le spécimen MNHN VIZ 27 (Fig. 14), par rapport au Mc III de G. robustum (MNHN PAM 141), l'indentation entre les facettes inférieure et dorsolatérale pour le magnum est plus profonde, la facette inférieure offre un contour ovoïde bien défini et son bord dorsal n'atteint pas le niveau 


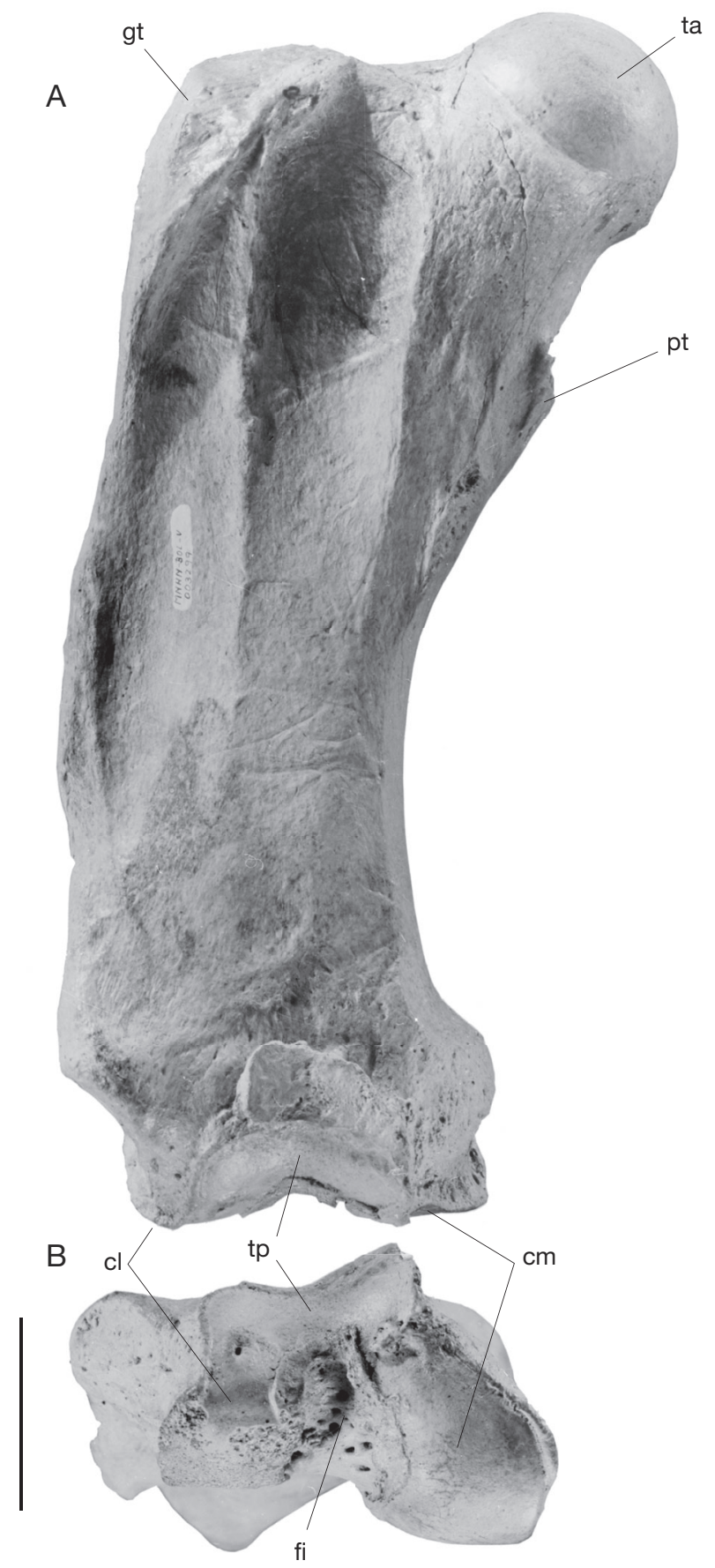

FIG. 16. - Fémur droit (MNHN-BOL V 3299) attribué à Simomylodon uccasamamensis n. gen., n.sp.: A, vue antérieure (proximale vers le haut); B, vue distale (antérieure vers le haut). Abréviations: cl, condyle latéral; $\mathbf{c m}$, condyle médial; fi, fosse intercondylienne; gt, grand trochanter; pt, petit trochanter; ta, tête articulaire; tp, trochlée patellaire. Échelle: $5 \mathrm{~cm}$. 

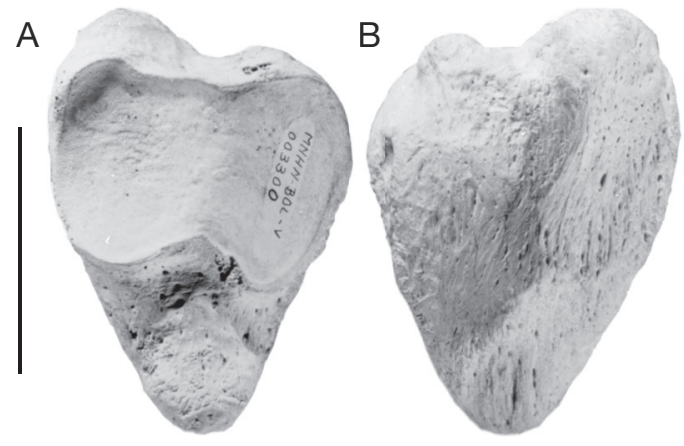

FIG. 17. - Rotule gauche (MNHN-BOL V 3300) attribuée à Simomylodon uccasamamensis n. gen., n.sp.: A, vue postérieure (proximale vers le haut); B, vue antérieure (proximale vers le haut). Échelle: $5 \mathrm{~cm}$.

$\mathrm{du}$ bord inférieur du condyle pour l'articulation avec le Mc II. Chez $G$. robustum, cette facette est de contour quadrangulaire et son bord supérieur dépasse le niveau du bord inférieur de l'articulation pour le Mc II. La facette dorsolatérale pour le magnum est endommagée.

Métacarpien IV. Par rapport au Mc IV de G. robustum (MNHN PAM 141), le Mc IV de Simomylodon n. gen. (spécimen MNHN VIZ 33 dépourvue de l'extrémité distale) présente une facette pour le $\mathrm{Mc} \mathrm{V}$ proportionnellement moins étendue ventralement et dont l'extrémité inférieure ne dépasse pas le niveau du bord ventral du corps de l'os. Par contre, cette facette est proportionnellement plus étendue en direction distale.

La facette pour l'unciforme est moins convexe aussi bien dorsoventralement que médiolatéralement. Elle présente également sur sa partie dorsale, une extension médiale empiétant sur la partie dorsolatérale de la facette pour le métacarpien III. Cette dernière est moins concave que chez G. robustum et ne présente pas à son extrémité médiale l'éversion de sa surface distincte du genre, caractère à mettre en rapport chez ce dernier avec l'extension, sur le Mc III, de la facette avec le Mc IV.

Fémur (Fig. 16). Le fémur possède la même forme générale que celui de Mylodon darwini mais surtout $G$. robustum. Par rapport à ces deux taxons, il est cependant moins massif, surtout au niveau du grand trochanter, dont la partie proximale est proportionnellement moins large par rapport à la longueur de l'os et la diaphyse plus allongée et plus grêle (Fig. 16A; Tableau 3).

La face latérale de la diaphyse présente, en dessous de la moitié de l'os, une zone plus proéminente que chez $G$. robustum mais moins développée et individualisée que le troisième trochanter de Pseudoprepotherium confusum. Le fémur offre un aspect général plus arqué (convexité latérale) que celui de G. robustum et beaucoup moins que chez Paramylodon harlani (Stock 1925) mais surtout que chez Pseudoprepotherium confusum (Hirschfeld 1985). Il présente la même torsion que chez ce dernier et la tête est projetée en avant par rapport au plan passant par les condyles et le grand trochanter.

Sur la face antérieure, sous le grand trochanter, une crête bien marquée sur les $2 / 3$ proximaux de la diaphyse devait séparer les muscles vastes latéral et intermédiaire (Fig. 16A). Très marquée également, la crête délimitant médialement ce dernier est parallèle à la première et s'étend du niveau du col jusqu’à la moitié de la diaphyse.

En vue distale, la fosse intercondylienne est moins profonde que chez $G$. robustum. La facette articulaire de la trochlée et les condyles sont unis (Fig. 16B) et sont conformes à ce qui peut être observé chez G. robustum.

Rotule (Fig. 17). La rotule de Simomylodon n. gen. est de forme triangulaire en vue antérieure ou postérieure et comparable à celle d'autres Mylodontinae tels que Glossotherium et Paramylodon. La rotule présente une double facette articulaire postérieure pour le fémur d'extension proximodistale (Fig. 17A). Les deux portions de cette facette, de proportions semblables, sont fortement concaves dans le sens proximodistal et légèrement transversalement. Cependant, la rotule est plus allongée et moins massive que chez Paramylodon

Tibia (Fig. 18). Cet os est semblable à son homologue chez Glossotherium robustum et Pseudoprepotherium et très différent de celui de Paramylodon, court et massif. Le tibia de Simomylodon n. gen. est cependant plus court et sa diaphyse, plus grêle, est moins élargie médiolatéralement que chez Glossotherium 

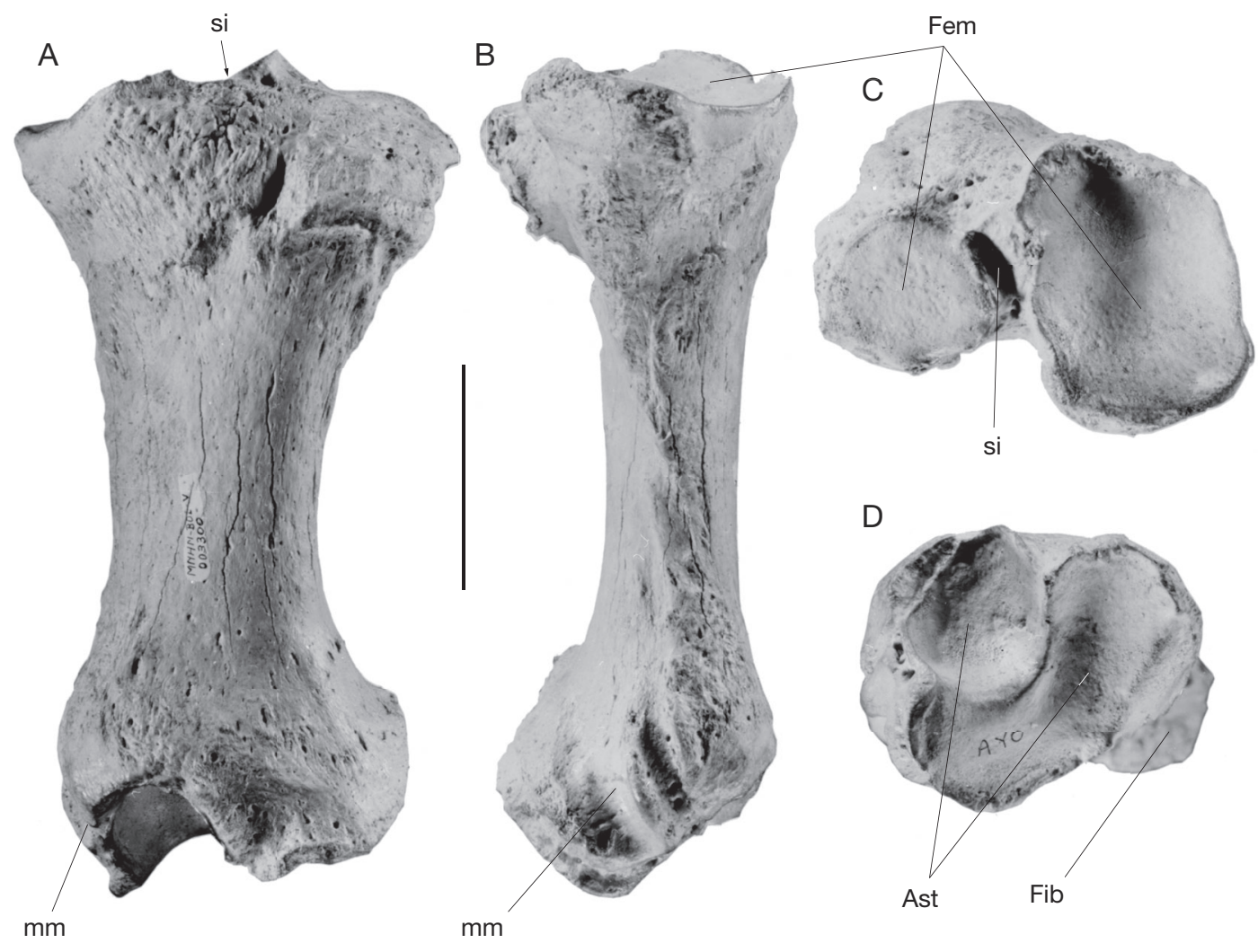

FIG. 18. - Tibia gauche (MNHN-BOL V 3300) attribué à Simomylodon uccasamamensis n. gen., n.sp.: A, vue antérieure (proximale vers le haut); B, vue médiale (proximale vers le haut); $\mathbf{C}$, vue proximale (antérieure vers le haut); $\mathbf{D}$, vue distale (antérieure vers le haut). Abréviations: Ast, astragale; Fem, fémur; Fib, fibula; $\mathbf{m m}$, malléole médiale; si, surface intercondylaire (échancrure rétrospinale). Échelle: $5 \mathrm{~cm}$.

(Fig. 18A, B; Tableau 3). Les épiphyses sont proportionnellement moins larges que chez ce dernier (Fig. 18C, D). La tubérosité latérale de la face postérieure de l'extrémité proximale, portant la facette fibulaire, est moins proéminente. L'échancrure rétrospinale est plus profonde et la partie antéro-externe de la facette fémorale médiale s'étend davantage en direction latérale (Fig. 18C).

Sur l'épiphyse distale, la partie concave de la surface articulaire astragalienne est légèrement plus profonde que chez G. robustum (Fig. 18D). L'arête la séparant de la surface pour le condyle latéral est nettement plus aiguë. Cette surface latérale est plus arquée autour de la facette médiale et la malléole médiale formant l'extrémité médiale de cette facette prolonge davantage l'arête intermédiaire.
Astragale (Fig. 19). L'astragale ressemble à celui de Glossotherium robustum. La différence la plus marquante est la présence, sur la face plantaire, d'un sulcus tali séparant les facettes ectale et sustentaculaire pour le calcanéum (Fig. 19A). La fusion de ces facettes et la disparition de ce sillon est un des traits dérivés les plus pertinents pour la diagnose des genres de Mylodontinae les plus spécialisés que sont Mylodon et Glossotherium. La facette ectale a la forme d'une "selle» dont le rayon de courbure longitudinal est plus faible que chez G. robustum.

La facette pour la fibula, de contour subcirculaire et séparée du condyle latéral et de la facette ectale chez $G$. robustum, s'étend du bord antérolatéral de ce condyle à la facette d'articulation avec le calcanéum chez Simomylodon n. gen. (Fig. 19B). 
La face latérale porte, derrière la facette d'articulation pour la fibula, une fossette de même forme que chez $G$. robustum.

La tête articulaire portant la facette pour le naviculaire est plus proéminente antérieurement par rapport au corps de l'os que chez Glossotherium, et le sillon séparant cette facette du bord antéromédial du condyle latéral est proportionnellement plus large que chez ce dernier genre. La facette pour le naviculaire est plus concave. Les arêtes émoussées séparant les diverses facettes portées par la tête articulaire sont plus marquées que chez $G$. robustum, chez lequel cette dernière a un aspect plus globuleux.

Le condyle interne, plus proéminent par rapport au condyle latéral, présente un aspect plus hémicylindrique que chez $G$. robustum où sa forme est plutôt "en quart de sphère" (Fig. 19B). La limite postérieure du condyle interne s'étend moins en direction médiale que chez Glossotherium, à la différence de la limite antérieure. En vue latérale, l'angle d'intersection des deux condyles au niveau de la gorge trochléaire est droit (Fig. 19B) comme chez Pleurolestodon alors qu'il est plutôt obtus chez Glossotherium ou Mylodon voire aigu chez Lestodon.

Cuboïde. Le cuboïde (MNHN AYO 128) diffère de celui de Glossotherium robustum (MNHN PAM 141) par une moindre extension médiolatérale de la facette d'articulation avec le calcanéum. Cette facette est de plus un peu décalée en direction latérale par rapport à celle de $G$. robustum.

L'extrémité antérieure de la facette pour l'astragale, concave comme $G$. robustum, forme cependant, entre les bords latéral et antéromédial, un angle plus aigu. Cette facette est de forme triangulaire, comme chez Pseudoprepotherium (Hirschfeld 1985) et fortement déversée antéromédialement.

Chez G. robustum, il n'y a pas de facette pour l'ectocunéiforme, à la différence de ce que l'on observe chez Pseudoprepotherium. Le bord antéromédial est droit jusqu'à la facette pour le Mt III, alors qu'il forme une indentation chez ce dernier genre. La facette pour le Mt III est proportionnellement plus petite et la distance la séparant de la facette pour le naviculaire est plus grande que chez Glossotherium. Chez $G$. robustum les facettes d'articulation pour les
Mt IV et V semblent ne former qu'une seule surface. Elles ont des inclinaisons différentes sur le cuboïde des spécimens d'Ayo Ayo, et l'angle obtus qu'elles composent entre elles forme une arête émoussée de direction transverse.

Métatarsien IV. Le Mt IV MNHN VIZ 27 incomplet (absence de l'extrémité distale) diffère de celui de G. robustum (MNHN PAM 141) par la moindre extension en direction plantaire de la facette d'articulation pour le Mt V. Sa limite ventrale se situe sur le même niveau que le bord ventral du corps de l'os et offre, en vue latérale, l'aspect d'un quart de disque, alors que, chez $G$. robustum, elle se prolonge jusqu'à l'extrémité du processus ventral de l'épiphyse proximale présentant un contour plutôt triangulaire.

La facette pour le cuboïde est légèrement convexe dans sa largeur alors qu' elle est plate chez $G$. robustum, ce qui donne à l'arête située entre les facettes pour le métatarsien IV et le cuboïde un aspect moins aigu. Elle est aussi nettement moins convexe dorsoventralement. Son bord ventral est arrondi, caractère en relation avec la forme du processus proximoventral, alors que, chez $G$. robustum, ce même bord forme un angle aigu.

\section{DISCUSSION}

Pleurolestodon dalenzae n. sp., du Miocène supérieur, et Simomylodon uccasamamensis n. gen., n. sp. du Pliocène de l'Altiplano bolivien, présentent des caractères proches des Mylodontidae-Mylodontinae tels que Glossotherium du Pléistocène d'Amérique du Sud et du Nord, Paramylodon du Rancholabréen et de l'Irvingtonien d'Amérique du Nord, Mylodonopsis du Pléistocène brésilien, Kiyumylodon du Hayquérien d'Uruguay ou Glossotheridium du Chapadmalalien d'Argentine et du Blanquien de Floride.

PLEUROLESTODON DALENZAE N. SP., SIMOMYLODON UCCASAMAMENSIS N. GEN., N. SP. ET LES MYLODONTINAE: CARACTÈRES RELATIFS AUX DENTS SUPÉRIEURES ET AU CRÂNE.

Les Mylodontinae sont généralement considérés comme le groupe frère des Scelidotheriinae. 
Pseudoprepotherium, du Colloncurien de Colombie, est classé parmi les Mylodontinae par Hirschfeld (1985) et Webb (1989). Les Scelidotheriinae sont considérés par Gaudin (2004) comme le groupe frère du clade constitué par Pseudoprepotherium, Octodontotherium et la sous-famille des Mylodontinae. Pseudoprepotherium présente plusieurs états de caractères considérés comme primitifs pour cette sous-famille: séries dentaires supérieures parallèles et symphyse mandibulaire en forme de gouttière, maxillaires allongés en avant de la $\mathrm{C}^{-}$, foramen entépicondylien présent, fémur relativement étroit, condyle médial de l'astragale de forme odontoïde mais peu élevé chez Pseudoprepotherium. Ces états de caractères sont probablement primitifs si on les compare à ceux observés chez des formes plus spécialisées comme Pleurolestodon, Simomylodon n. gen., Mylodonopsis, Glossotheridium, Glossotherium, Thinobadistes (Hemphillien du sud des États-Unis) ou Lestodon (Lujanien).

De nombreux caractères de morphologie dentaire rapprochent Simomylodon $\mathrm{n}$. gen. et Pleurolestodon de Glossotherium, Glossotheridium et Kiyumylodon.

La C- est absente sur le spécimen type de Pseudoprepotherium confusum, son alvéole est intacte. Laspect caniniforme de la première dent supérieure de Pleurolestodon et Simomylodon n. gen. pourrait correspondre à un état plésiomorphe également identifié chez le genre santacruzien Nematherium (Scott 1904; Simpson 1941); il est rapproché des Scelidotheriinae par McDonald \& Perea (2002) et reconnu par McKenna \& Bell (1997) et Gaudin (2004) comme le groupe frère des «Mylodontoides".

La C- est de taille légèrement inférieure à la M1 chez Pleurolestodon et Simomylodon n. gen., comme d'ailleurs chez Mylodonopsis, Glossotheridium et Pseudoprepotherium. Cette différence de taille entre C- et M1, présente chez les Scelidotheriinae et Pseudoprepotherium, représenterait un caractère primitif de la sous-famille (à condition que Pseudoprepotherium appartienne bien aux Scelidotheriinae).

La C- est plus développée chez les Lestodontini. Chez certains représentants du genre Glossotherium, la C- est très forte, ce qui a conduit Gervais $\&$ Ameghino (1880) à attribuer ces spécimens au genre Pseudolestodon, dont Cabrera (1936) réfute

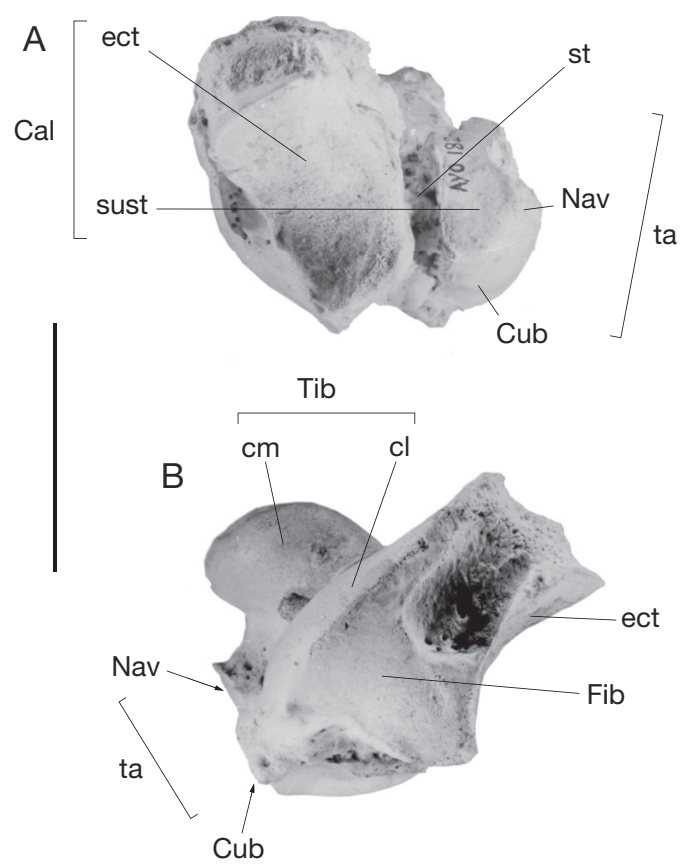

FIG. 19. - Astragale gauche (MNHN AYO 182) attribué à Simomylodon uccasamamensis n. gen., n.sp. : A, vue plantaire (postérieure vers la droite); B, vue latérale (postérieure vers le haut). Abréviations: Cal, calcanéum; cl, condyle latéral; cm, condyle médial; Cub, cuboïde; ect, facette ectale pour le calcanéum; Fib, fibula; Nav, naviculaire; st, sulcus tali; sust, facette sustentaculaire pour le calcanéum; ta, tête articulaire; Tib, tibia. Échelle: $5 \mathrm{~cm}$.

pourtant la validité. Nous acceptons cependant la thèse de Cabrera sur les caractères diagnostiques de Pseudolestodon qui évoqueraient des traits relatifs à l'âge ou au sexe des spécimens en dépit d'une tentative de réhabilitation de Pseudolestodon comme sous-genre de Glossotherium par Parodi Bustos (1974). Une C- de grande taille chez les Lestodontini et chez Glossotherium et Mylodonopsis est probablement un caractère dérivé. Au vue de ces observations, Pleurolestodon et Simomylodon n. gen. seraient moins spécialisés que Glossotherium. Enfin la C-, réduite à absente chez Glossotherium, disparaît définitivement chez Mylodon.

Selon Webb (1989), la réduction de la C- dans la lignée de Mylodontinae Pleurolestodon-Mylodon par rapport aux Lestodontini serait une réversion permettant de diagnostiquer les Mylodontinae; Glossotherium présentant, avec une C- de grande 
taille, l'état plésiomorphe du caractère de cette sous-famille. Cette réduction de la C- permettrait de placer Pleurolestodon et Simomylodon n. gen. parmi les Mylodontidae plus spécialisés que Glossotherium. Ce dernier conserverait de l'ancêtre commun aux Lestodontini et aux Mylodontinae une C- de taille supérieure à la M1. Toutefois, les caractères ostéologiques (discutés ci-dessous) permettront de faire l'hypothèse que Lestodon et Glossotherium ont atteint indépendamment des états comparables de certains caractères, tels que la taille de la C-. Dans ce cas, Simomylodon n. gen. et vraisemblablement Pleurolestodon (l'état de conservation du matériel ne nous a pas permis d'observer les caractères ostéologiques) sont des Mylodontinae moins dérivés que Glossotherium.

Chez Simomylodon n. gen. et Pleurolestodon, la M2 possède une section en forme de triangle rectangle, d'angle droit en position antérolinguale et un sillon lingual, comme chez Glossotheridium, Glossotherium et Paramylodon. Cette configuration qui existe aussi chez Thinobadistes, signifierait sa disparition ultérieure chez Lestodon, dont les dents possèdent des sections très arrondies. La M2 des Scelidotheriinae est de section triangulaire, très étirée labiolingualement. Chez Pseudoprepotherium, la M2 est très arrondie quoique la face linguale, plus aplatie que la face labiale, possède un léger sillon lingual. Elle serait donc une autapomorphie de ce taxon (Webb 1989). La forme est plutôt arrondie chez Mylodon darwini dont la M2 est de section grossièrement triangulaire avec les arêtes arrondies et la face interne déprimée (Kraglievich 1934), ce qui correspondrait à une simplification secondaire du schéma observé chez Glossotherium et genres affins (y compris Pleurolestodon et Simomylodon n. gen.). Cependant, chez Pleurolestodon, l'allongement de la M2 par rapport à la M3 est beaucoup plus important que chez les autres genres, ce qui indiquerait une autapomorphie du genre.

Bien que possédant une section semblable à celle de la M2 chez la plupart des Mylodontinae, la M3 est normalement plus comprimée antéropostérieurement. Ce caractère assez général est observé chez Pseudoprepotherium, Simomylodon n. gen., Pleurolestodon, Paramylodon, Thinobadistes, ainsi que pour le type de Glossotheridium chapadmalense et
Glossotherium. La M3 de Glossotheridium chapadmalense (UF 10922) de Floride, est en revanche moins comprimée antéropostérieurement que la M2 (Robertson 1976: fig. 2), cas probablement unique chez les Mylodontinae et qui permet de s'interroger sur l'attribution de ce spécimen au taxon Glossotheridium chapadmalense. Mylodon possède une M3 de section triangulaire dont la face linguale porte un sillon. Chez Pseudoprepotherium, cette dent, comme la M2, est de contour arrondi avec une face linguale aplatie, marquée d'un léger sillon. Une M2 présentant une forme arrondie et non comprimée antéropostérieurement correspondrait à une autapomorphie.

Les Mylodontinae présentent une dilatation dorsoventrale du museau absente chez les Lestodontini. Une telle dilatation est particulièrement marquée chez Simomylodon n. gen., Pleurolestodon, Glossotherium et Mylodon.

L'absence de diastème entre la C- et la M1 peut être considérée comme la condition primitive retrouvée chez Pseudoprepotherium et les Scelidotheriinae. Glossotherium robustum et $G$. wegneri possèdent un diastème de faible longueur entre la C- et la M1. Pleurolestodon, Paramylodon, Mylodonopsis, Simomylodon n. gen., Glossotheridium et Paramylodon en sont dépourvus alors que Mylodon a perdu la dent la plus antérieure (qui correspond à l'état le plus dérivé). Un tel diastème caractérise le genre Glossotherium ( $G$. robustum et $G$. wegneri), les Lestodontini tels que Lestodon, Thinobadistes et probablement certains autres genres mal connus comme Ranculcus et Sphenotherus. Ce caractère est considéré par Webb (1989) comme une synapomorphie des "Lestodontini-Mylodontini». La réduction et la disparition du diastème chez Pleurolestodon et Paramylodon pourraient ainsi être considérées comme une réversion chez les Mylodontinae. L'absence de diastème entre la C- et la M1, l'élargissement du museau et la divergence des séries dentaires chez Simomylodon n. gen. et Pleurolestodon, permettent de classer ces genres parmi les Mylodontinae, dont Glossotherium serait l'un des taxons les moins dérivés quant à la réversion qui caractérise ce groupe.

Cependant, l'interprétation de Webb (1989) sur la réduction du diastème chez les Mylodontinae ne semble pas prendre en compte les caractères 
ostéologiques postcrâniens de ce clade. L'absence de diastème notamment chez Pleurolestodon et Simomylodon n. gen., représenterait l'état primitif du caractère alors qu'un diastème entre la $\mathrm{C}$ - et la $\mathrm{M} 1$ se serait développé indépendamment chez Glossotherium et les Mylodontidae Lestodontini.

Le maxillaire des genres Simomylodon n. gen. et Pleurolestodon, comme chez Glossotherium et Glossotheridium, ne s'étend pas en avant de la C-. Chez Lestodon et Thinobadistes, l'extrémité antérieure des maxillaires est arrondie et s'étend très peu en avant de la première paire de molaires. Par contre, chez Pseudoprepotherium et Urumacotherium et les Scelidotheriinae comme Proscelidodon Bordas, 1935, Scelidodon et Scelidotherium, le maxillaire se prolonge en avant de la C-, ce qui correspondrait à l'état primitif chez les Mylodontinae. Comme mentionné précédemment chez Mylodon, la C- a disparu.

L'élargissement du museau et la divergence des séries dentaires supérieures sont des caractères probablement liés chez les Mylodontinae. Chez Pseudoprepotherium, l'écartement des séries dentaires supérieures est très peu marqué alors que chez les Scelidotheriinae il est quasiment nul, ce qui correspondrait à la condition primitive la famille. Simomylodon n. gen. et Pleurolestodon montrent un élargissement du museau vers l'avant et une divergence assez régulière de l'arrière vers l'avant des dents supérieures. Cette morphologie est caractéristique des Mylodontinae, bien que très peu marquée chez Pseudoprepotherium. Elle atteint son paroxysme chez Glossotherium wegneri et les Mylodontinae Lestodontini. Cette probable synapomorphie est retrouvée chez Pleurolestodon, Urumacotherium campbelli (Frailey 1986; Mones 1986; Negri \& Ferigolo 2004), Pleurolestodon dalenzae n. sp., mais est absente chez la forme pléistocène Mylodon.

L'élargissement du museau chez Lestodon (Lujanien) et Thinobadistes (Hemphillien) est lié à un important déplacement vers l'avant des C- par rapport aux autres dents, consécutif à l'existence d'un long diastème entre C- et M1. Un diastème comparable se retrouve chez Ranculcus (Huayquérien) et est considéré comme une synapomorphie liant ces trois genres (Hirschfeld 1985; Webb 1989). Un fort élargissement du museau et un court diastème s'observent également chez Glossotherium.

Chez Simomylodon n. gen. et Pleurolestodon dalenzae n. sp., la distance mesurée entre les faces internes des C- est supérieure au double de celle mesurée entre les M4. La divergence y est plus prononcée que chez Pleurolestodon acutidens, où la distance entre les C- est inférieure au double de celle existant entre les M4. Elle est également plus importante que chez le spécimen type de Glossotheridium chapadmalense, où la distance séparant les C- est égale au double de celle mesurée entre les M4. Il semblerait cependant, au vu de la photographie du palais du spécimen UF 10922 de Glossotheridium chapadmalense (Robertson 1976: fig. 2), que la distance entre les C- soit plus du double de celle existant entre les M4. Chez Simomylodon n. gen. et Pleurolestodon cette divergence des séries dentaires supérieures est plus faible que celle mesurée chez Glossotherium robustum et les Lestodontinae. Si l'élargissement du museau et la divergence des séries dentaires constituent l'état dérivé du caractère, Simomylodon n. gen. et Pleurolestodon doivent être considérés, sur ce point, comme moins spécialisés que Glossotherium et les Lestodontini.

De même que la diminution de taille de C- peut représenter une réversion chez les Mylodontinae, Webb (1989; voir ci-dessus) considère implicitement, en faisant du groupe Pleurolestodon-Mylodon le groupe frère de Glossotherium et de ce genre le taxon plésiomorphe des "Mylodontini", qu'un rétrécissement du museau et une réduction de la divergence des séries dentaires diagnostiquent les "Mylodontini» au même titre que la réduction de la C-. Cette hypothèse peut être rapportée à la morphologie de l'extrémité antérieure étroite et pointue du crâne observée chez Mylodon darwini (Lydekker 1894). Le museau de ce taxon est dorsoventralement dilaté, légèrement élargi en avant des M1 (les C- ont disparu), puis rétréci en forme d'arc lancéolé (Ameghino 1889). La disparition des $\mathrm{C}^{-}$a donc vraisemblablement entraîné, à partir d'une morphologie proche de celle de type "Glossotherium», une modification de la partie antérieure du museau. Le reste du squelette de cet animal est tout-à-fait comparable à Paramylodon harlani où la tendance marquée à la réduction des 
C- serait pour Webb (1989) une synapomorphie des «Mylodontini ».

Cependant, il est peu probable que ces réversions soient caractéristiques de l'ensemble des Mylodontinae et que la morphologie crânio-dentaire de Glossotherium soit directement héritée du dernier ancêtre commun à ce taxon et aux Lestodontinae. De telles réversions caractérisent plutôt un clade regroupant Glossotherium, Paramylodon et Mylodon.

En fait, les implications de ces réversions sont soumises à la façon de considérer certains des états de caractères, similaires chez Glossotherium et les Mylodontinae Lestodontini : la présence d'un diastème entre la $\mathrm{C}^{-}$et la $\mathrm{M} 1$, la divergence des séries dentaires, l'élargissement du museau, ainsi que la taille relativement développée de la C-, sont considérés par Webb (1989), comme des synapomorphies. La réduction de la divergence des séries dentaires, le rétrécissement du museau et la réduction de la Csont considérés chez les Mylodontinae comme des réversions. L'hypothèse alternative qui supporterait l'acquisition indépendante chez Glossotherium et les Mylodontinae Lestodontini, de la divergence des séries dentaires, de l'élargissement du museau et de la taille relativement développée de la C-, s'appuie sur l'apparition tardive (Pléistocène) de Glossotherium au sein des Mylodontinae par rapport à la lignée des Lestodontini (Thinobadistes apparaît au Miocène supérieur en Amérique du Nord) mais également sur l'existence, entre le Miocène supérieur et le Pléistocène, de Pleurolestodon dalenzae n. sp. et Simomylodon uccasamamensis n. gen., n. sp., formes comparables à Glossotherium et présentant avec ce genre plusieurs synapomorphies (dilatation dorsoventrale du museau, développement d'une pelle symphysaire présentant une constriction en avant des $\mathrm{C}_{\text {, }}$ fémur large, condyle médial odontoïde de l'astragale plus élevé), mais cependant moins dérivées quant aux caractères concernant le crâne et les dents supérieures. Si l'on considère la fusion des facettes de l'astragale pour le calcanéum comme une synapomorphie des genres Glossotherium et Mylodon, l'existence de ces formes mio-pliocènes, chez qui le sulcus tali demeure, indique que les similitudes dans les morphologies crânienne et dentaire de Glossotherium et des Lestodontini pourraient être consécutives à un phénomène de parallélisme évo- lutif plutôt qu'à des états de caractères hérités d'un ancêtre commun qui leur soient propres.

Les genres Simomylodon n. gen. et Pleurolestodon possèdent des caractères crâniens caractéristiques des Mylodontidae (réduction des maxillaires en avant de C-', dilatation du museau et écartement des séries dentaires d'arrière en avant). La dilatation dorsoventrale du museau permet de les classer parmi les Mylodontinae mais de les exclure des «Lestodontines» dont ils ne présentent pas les synapomorphies (élargissement maximal du museau, symphyse mandibulaire élargie en pelle sans rétrécissement en avant des $\mathrm{C}_{-}$).

Leur dentition supérieure, semblable à celle des genres Mylodonopsis, Glossotherium et Glossotheridium, ne présente pas de synapomorphies notables, comme l'existence d'un diastème entre la C- et M1 et l'existence d'une C- de grande taille typiques de certains Glossotherium, ou la réduction, voire la disparition, de C- chez Paramylodon.

Une fois admises les implications des réversions (réduction des C-, du diastème entre C- et $\mathrm{M} 1$, de la divergence des séries dentaires, de l'élargissement du museau) utilisées par Webb (1989) restreintes à Paramylodon et Mylodon, le fort élargissement du museau, l'importante divergence des dents vers l'avant et la grande taille de C- doivent être considérés comme des autapomorphies de Glossotherium. Un élargissement du museau, une moindre divergence des dents et une C- de taille relativement réduite permettent de classer Pleurolestodon et Simomylodon n. gen. parmi les Mylodontinae, moins dérivés cependant que le groupe Glossotherium-Mylodon.

Position de PLEUROLESTODON DALENZAE N. SP. ET SIMOMYLODON UCCASAMAMENSIS N. GEN., N. SP. AU SEIN DES MYLODONTINAE

Pleurolestodon dalenzae n. sp. est connu par un crâne appartenant à un Mylodontinae (MNHN-BOL V 3348), comme l'indique notamment la dilatation dorsoventrale du museau, présentant les caractéristiques majeures de Pleurolestodon mais cependant différent de $P$. acutidens (Rovereto 1914). Il possède des dents supérieures de même forme générale que Glossotherium, Mylodonopsis, Glossotheridium et Simomylodon n. gen. 
Une C- de petite taille est sans doute un caractère plésiomorphe car présent chez les Scelidotheriinae et Pseudoprepotherium. Pleurolestodon semble être un Mylodontinae moins dérivé que Glossotherium.

Un sillon lingual très marqué sur la M2 et l'allongement important de cette dent pourraient être des autapomorphies de cette espèce de Pleurolestodon, qui la différencient de Simomylodon n. gen. et des autres Mylodontinae. Cette spécialisation de la M2 de Pleurolestodon dalenzae n. sp. n'est pas un caractère suffisant pour situer ce taxon dans l'ascendance directe de Simomylodon n. gen., qui est son descendant chronostratigraphique immédiat et qui présente une M2 identique à celle des autres membres des Mylodontinae, triangulaire comme la M3 mais moins comprimée antéropostérieurement et non étirée comme chez Pleurolestodon.

Les prémaxillaires ne présentent aucune tendance au raccourcissement de leur extrémité antérieure médiane, alors que chez Simomylodon n. gen., le bord incisif du prémaxillaire est presque perpendiculaire au plan sagittal.

L'élargissement du palais est proportionnellement supérieur à celui de Pleurolestodon acutidens et Glossotheridium, équivalent à celui de Simomylodon n. gen. et moindre que chez Glossotherium robustum. Ces observations permettent de considérer Pleurolestodon dalenzae n. sp. comme un taxon plus spécialisé que Pleurolestodon acutidens et Glossotheridium chapadmalense. La dilatation dorsoventrale du museau, moins importante que chez Glossotherium, serait également un caractère dérivé par rapport à ce qui peut être observé chez Pseudoprepotherium et Glossotheridium. Ainsi Pleurolestodon dalenzae n. sp. pourrait être un Mylodontinae plus dérivé que $P$. acutidens et Glossotheridium chapadmalense mais moins évolué que Glossotherium. La possibilité de réversions chez les Mylodontinae (réduction de la taille des C-, divergence des séries dentaires et, peut-être, dilatation du museau), considérées par Webb (1989) comme diagnostiques des «Mylodontini ", ne peut être écartée par l'étude des pièces squelettiques postcrâniennes de Pleurolestodon dalenzae n. sp., comme c'est le cas pour Simomylodon uccasamamensis n. gen., n. sp. Toutefois, les similitudes entre les crânes de Simomylodon n. gen. et Pleurolestodon laissent penser que ce dernier est un Mylodontinae, moins dérivé cependant que le groupe Glossotherium-Mylodon.

La présence d'un Mylodontidae-Mylodontinae de taille modeste dans le Pliocène de l'Altiplano, près d'Ayo Ayo, a été signalée par Hoffstetter et al. (1971). Comme les faunes d'Ayo Ayo et de Viscachani ont été considérées comme semblables (thèse à laquelle nous souscrivons), les restes de Mylodontinae provenant de ces deux localités ont tacitement été attribués à un même taxon. L'examen de l'humérus, du fémur et de la mandibule permit à Hoffstetter et al. (1971) de classer cet animal au sein des Mylodontinae. Ces auteurs signalent en outre que certains traits relatifs aux dents et aux métapodes suggèrent un nouveau genre. Par la suite, Hoffstetter et al. (1972) signalent la présence de «Mylodontidés de petite taille» dans le Pliocène de Pomata, au-dessus de la Toba 76 sans préciser leur degré de similitude avec le Mylodontinae d'Ayo Ayo-Viscachani. Ils mentionnent le caractère contemporain des faunes de ces deux gisements.

Lavenu $(1984,1986)$ indique l'existence dans la Formation Remedios, de Mylodontinae «identiques à la forme pliocène d'Ayo Ayo et Viscachani » (ce matériel, aujourd'hui disparu, n’a pu être étudié).

Enfin, Hoffstetter (1986) précise que le Mylodontinae des gisements pliocènes de l'Altiplano diffère de Glossotheridium.

Le genre Glossotheridium a été créé par Kraglievich (1934) pour Eumylodon chapadmalensis Kraglievich, 1925. Robertson (1976), identifie le Mylodontinae (UF 10922) du Blanquien de Floride (provenant du site Haile XV A), comme Glossotherium chapadmalensis (= Glossotheridium chapadmalense). Pour cet auteur il s'agit d'une petite espèce étroitement apparentée à Paramylodon harlani et G. robustum, mais qui en diffère par la nature de la dentition antérieure et la taille générale. Glossotherium chapadmalensis s'apparente à Paramylodon harlani et à G. robustum par la morphologie de la plupart des éléments postcrâniens, avec des différences mineures au niveau de la main et du pied. Robertson (1976) conclut que $G$. chapadmalensis est chronologiquement, géographiquement et morphologiquement un ancêtre probable de Paramylodon harlani et G. robustum. 
Simomylodon n. gen. est caractérisé par des prémaxillaires formant un bord incisif droit qui ne se projettent pas vers l'avant près du plan sagittal, comme c'est le cas chez Glossotherium robustum, G. wegneri ou Pleurolestodon dalenzae n. sp. Chez le type de Glossotheridium chapadmalense comme sur le spécimen UF 10922 de Floride (Robertson 1976), les prémaxillaires n'ont pas été conservés. Cependant, chez le type, le développement des processus palatins antérieurs des maxillaires en avant du niveau des C- plaide en faveur d'une disposition des prémaxillaires semblable à celle observée chez Glossotherium. Chez Simomylodon n. gen., ces processus ne dépassent que de fort peu la droite passant par le bord antérieur des C-. Cette dernière morphologie semble caractéristique de ce nouveau genre. Par contre, l'extension latérale du prémaxillaire se soude au maxillaire en avant de C-. Cette disposition a été décrite par Webb (1989) dans son cladogramme des Mylodontidae dans les termes suivants: «subcircular premaxillary expanded horizontally, but loses vertical ramus». Cet état de caractère correspondrait à une synapomorphie du groupe Glossotherium-Glossotheridium-MylodonopsisPleurolestodon-Mylodon, groupe dont font partie les deux nouveaux taxons Simomylodon uccasamamensis n. gen., n. sp. et Pleurolestodon dalenzae n. sp.

La morphologie particulière des prémaxillaires de Simomylodon $\mathrm{n}$. gen. et la brièveté vers l'avant des processus palatins des maxillaires confirment la distinction entre Glossotheridium et Simomylodon n. gen. signalée par Hoffstetter (1986). En ce qui concerne la taille des individus, Simomylodon uccasamamensis n. gen., n. sp. et Pleurolestodon dalenzae n. sp. sont nettement plus petits que Glossotheridium chapadmalense.

Simomylodon uccasamamensis n. gen., n. sp. et P. dalenzae n. sp. présentent une divergence des séries dentaires supérieures plus importante que Glossotheridium. Le parallélisme des séries dentaires représenterait l'état plésiomorphe du caractère observé chez Pseudoprepotherium, Mylodontinae primitif selon Hirschfeld (1985), et chez les Scelidotheriinae. Mais le degré de divergence des séries dentaires supérieures et l'élargissement du museau sont moindres chez Simomylodon n. gen. que chez Glossotherium, ce qui indiquerait un stade moins dérivé que chez ce dernier taxon, que des caractères ostéologiques relient préférentiellement à Paramylodon et Mylodon.

\section{CARACTÈRES DENTAIRES (DENTS INFÉRIEURES)}

ET MANDIBULAIRES

Chez Simomylodon n. gen., Glossotheridium et Glossotherium, la C. montre une section arrondie, aplatie sur la face linguale marquée d'un léger sillon. Par contre, Pleurolestodon dalenzae n. sp. possède

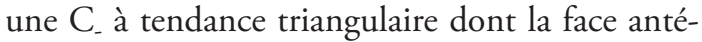
rieure montre un léger sillon en son milieu et une face linguale plus fortement concave que chez les autres Mylodontinae (Kraglievich 1921). La C_ de Promylodon (Huayquérien) présente également un sillon antérieur (Paula Couto 1979). Ranculcus, du Huayquérien d'Argentine, possède une $\mathrm{C}_{\text {_ }}$ "subcilíndrincotriangular» dotée de deux petits sillons sur les faces antérieure et médiale. L'alvéole de cette dent sur la mandibule du type de Sphenotherus zavaletianus Ameghino, 1891, du Huayquérien d'Argentine, montre qu'elle devrait être dépourvue de sillon antérieur. Par contre, la face médiale est concave. Chez Pseudoprepotherium, la $\mathrm{C}_{-}$, de section ovale, est aplatie postérolingualement avec une légère indentation du bord antérolabial (Hirschfeld 1985) comme chez Kiyumylodon lecuonai (Rinderknecht, Perea \& McDonald, 2007). Une C_de section arrondie avec la face antérieure dépourvue de sillon est présente chez Simomylodon n. gen., Glossotherium et Glossotheridium. C'est un état probablement dérivé par rapport à celui où la dent, grossièrement triangulaire, est munie d'un sillon sur sa face antérieure, comme chez Pseudoprepotherium confusum, Pleurolestodon dalenzae n. sp. et Ranculcus scalabrinianus Ameghino, 1891.

La $\mathrm{m} 1$ est de contour triangulaire, arrondi chez Nematherium (Scelidotheriinae primitif), Kiyumylodon et Pleurolestodon, caractère qui correspondrait à la condition primitive chez les Mylodontinae. Un fût de contour arrondi avec une légère indentation linguale chez Pseudoprepotherium (Hirschfeld 1985) correspondrait à une autapomorphie de ce genre, dont toutes les dents ont tendance à acquérir une section arrondie. Il en est de même chez Lestodon, où la dent est de section plus elliptique. Chez Pleurolestodon dalenzae n. sp., Simomylodon 
uccasamamensis n. gen., n. sp. et Glossotheridium chapadmalense, la $\mathrm{m} 1$ est de section triangulaire avec une face linguale marquée d'un sillon et d'une face postérolabiale convexe. C'est le premier état dérivé des Mylodontinae. Chez Glossotherium wegneri, s'ajoute un sillon sur la face postérolabiale alors que chez $G$. robustum, un troisième sillon apparaît sur la face antérieure. Ces deux derniers états correspondent à des conditions dérivées de celles rencontrées chez Pleurolestodon, Simomylodon n. gen. et Glossotheridium. Chez Ranculcus scalabrinianus, la $\mathrm{m} 1$ présente un contour très particulier en vue occlusale. En effet chez ce taxon la face antérieure est munie d'un sillon bien marqué, sa face linguale est légèrement concave, la partie labiale très convexe et le contour de la section plus campaniforme que triangulaire.

La section de la $\mathrm{m} 2$ de forme triangulaire observée chez les Scelidotheriinae et chez Thinobadistes souligne l'état primitif de ce caractère. L'état correspondant à une $\mathrm{m} 2$ de section arrondie, marquée d'un léger sillon lingual, serait alors une autapomorphie de Pseudoprepotherium confusum (Webb 1989). Cette section arrondie, que l'on retrouve chez Lestodon et Kiyumylodon, permet aussi de penser qu'il pourrait s'agir d'une synapomorphie des Mylodontinae. Cependant, la simplification des dents supérieures précédemment évoquée chez Lestodon suggérerait l'existence d'une convergence entre ce dernier (dont la $\mathrm{m} 2$ aurait évolué à partir de la forme généralisée à section triangulaire comme chez Thinobadistes) et Pseudoprepotherium. Un autre état dérivé, autapomorphe, correspondrait, chez Sphenotherus zavaletianus, à un allongement du grand axe de la section elliptique de la dent, principalement au niveau de la partie antérolabiale. Enfin, un troisième état dérivé se trouve chez Simomylodon n. gen., Pleurolestodon acutidens, Ranculcus scalabrinianus, Promylodon paranensis Ameghino, 1883 et Glossotherium: il s'agit de l'existence d'une $\mathrm{m} 2$ qui, si son grand axe est bien allongé antérolabialement postérolingualement, revêt, en section, une forme "parallélogrammatique rhombique» et non plus elliptique.

La m3 bilobée est caractéristique des Mylodontoidea, comme chez les Scelidotheriinae, Thinobadistes et Pleurolestodon qui possèdent une $\mathrm{m} 3$ dont la face antérieure est concave et la face postérieure convexe. Chez le type de Ranculcus scalabrinianus, Kraglievich (1921), se fondant sans doute sur la forme de l'alvéole (la partie antérieure de cette dent est abîmée), affirme que la configuration de la dent est comparable à celle de Pleurolestodon acutidens. Elle possède une face postérieure plus aplatie que chez Pseudoprepotherium confusum et ressemblerait à la m3 utilisée par Ameghino (1891) pour créer le taxon Sphenotherus paranensis Ameghino, 1891. Chez Simomylodon n. gen. et Glossotherium, la face antérieure de la m3 est concave et la face postérieure aplatie. L'isthme joignant les deux lobes est plus allongé et sa face labiale porte un troisième lobe intermédiaire, peu développé en regard des lobes primitifs, inexistant chez les genres précédemment cités. Chez Promylodon et Lestodon, la section de la $\mathrm{m} 3$ prend la forme d'un « 8 ». Chez ces deux genres et chez Pseudoprepotherium, les faces antérieure et postérieure de la dent sont convexes. Chez Sphenotherus zavaletianus, l'alvéole de la m3 présente deux lobes arrondis, ce qui suggère que les faces antérieure et postérieure étaient également convexes. Nous disposons ainsi, pour la m3, d'un morphocline où plusieurs caractères permettent de mieux appréhender l'histoire des Mylodontinae. L'état primitif de la m3 semble correspondre à une dent dont le lobe antérieur est aplati à légèrement concave, et la face postérieure convexe comme chez Ranculcus scalabrinianus, Sphenotherus paranensis et les Scelidotheriinae. Chez Pseudoprepotherium et Lestodon, les deux lobes sont arrondis, réunis par un isthme court et la $\mathrm{m} 3$ prends la forme d'un « 8 ». Ce caractère doit être corrélé avec la simplification et l'arrondissement des dents déjà signalés pour ces deux genres. Une condition similaire existe avec Promylodon et Sphenotherus. Chez Pseudoprepotherium et Lestodon, il s'agit sans doute d'une convergence puisque les Scelidotheriinae, Thinobadistes, Ranculcus et le groupe Pleurolestodon-Simomylodon n. gen.Glossotherium-Glossotheridium-Mylodon-Kiyumylodon possèdent une $\mathrm{m} 3$ dont la face antérieure est plane à concave, ce qui semble être la condition primitive. L'allongement de la m3 évoque une synapomorphie liant les formes Lestodontines (Thinobadistes et Lestodon) et les Mylodontinae (tels que Pleurolestodon, Simomylodon n. gen., Glossotherium, Glossotheridium, 
Kiyumylodon et Mylodon) (Webb 1989). Les Mylodontinae sont caractérisés par une $\mathrm{m} 3$ dont le lobe postérieur n'est pas saillant labialement. Un dernier état dérivé correspond à une dent dont la face antérieure est très concave et la face postérieure convexe. L'isthme est long et porte parfois labialement une faible gibbosité que Kraglievich (1921) nomme troisième lobe intermédiaire. Une telle morphologie s'observe chez Glossotheridium, Simomylodon n. gen., Glossotherium et Paramylodon.

Les caractères relatifs aux dents inférieures de Simomylodon n. gen. corroborent les résultats obtenus à partir des caractères crâniens. Parmi les Mylodontinae, Simomylodon n. gen. serait plus dérivé que Pseudoprepotherium, les Lestodontines et Pleurolestodon, en raison de la forte concavité du bord antérieur de $\mathrm{m} 3$, du développement uniquement lingual de cette même dent et de la disparition du sillon antérieur sur la C. En revanche, l'absence de sillons sur les faces postérolabiale et antérieure de la $\mathrm{m} 1$ indique que Simomylodon $\mathrm{n}$. gen. est à un stade évolutif moins dérivé que le genre Glossotherium.

La morphologie de la mandibule et l'existence d'une pelle symphysaire rapprochent davantage Simomylodon n. gen. de Glossotheridium, du Chapadmalalien d'Argentine et du Blanquien de Floride, de Glossotherium du Quaternaire sud- et nord-américain et Kiyumylodon, du Huayquérien d'Uruguay que de Pseudoprepotherium, du Colloncurien de la faune de La Venta en Colombie. La symphyse a, d'une manière générale dans la famille des Mylodontidae, une forme allongée et étroite bien illustrée chez les Scelidotheriinae et Pseudoprepotherium. Des états dérivés apparaissent chez Pleurolestodon, groupe formé par Thinobadistes-Lestodon et dans l'ensemble Simomylodon n. gen.-Glossotherium-GlossotheridiumKiyumylodon-Mylodon où la symphyse est plus large que la série dentaire (Webb 1989). Un élargissement subséquent est réalisé, sans doute par convergence (Webb 1989), chez Thinobadistes et Lestodon d'une part et chez les genres Simomylodon n. gen., Kiyumylodon, Glossotherium et Glossotheridium d'autre part, chez qui la symphyse mandibulaire est élargie en pelle. Chez Pseudoprepotherium confusum, du Colloncurien, et chez les Scelidotheriinae tels que Scelidodon, Scelidotherium et Proscelidodon, la symphyse mandibulaire est étroite en forme de gouttière.
Chez Pleurolestodon acutidens du Huayquérien, elle prend la forme d'un "pico de jarra» («bec de cruche») selon l'expression de Rovereto (1914) mais est plus large que chez Pseudoprepotherium (Webb 1989).

Chez les autres Mylodontinae, la symphyse mandibulaire est très élargie, aboutissement d'une tendance évolutive qui semble s'être réalisée dans les groupes Thinobadistes-Lestodon et Simomylodon n. gen.-Glossotherium-Glossotheridium-Kiyumylodon. Mylodon appartient vraisemblablement à ce dernier groupe mais comme la symphyse mandibulaire de Mylodon darwini est allongée et non élargie (Lydekker 1894), cela pourrait correspondre à une régression.

Lorsque la symphyse mandibulaire est élargie en pelle, les genres qui disposent de ce caractère présentent une gradation. En effet, chez les Mylodontinae Lestodontini (tels que Sphenotherus-Ranculcus-Thinobadistes-Lestodon; Hirschfeld 1985) et plus spécialement chez Sphenotherus zavaletianus du Huayquérien, la symphyse, bien qu'imparfaitement soudée, présente un début d'élargissement. Pour des raisons touchant à la disposition des dents sur la mandibule, Sphenotherus est considéré comme proche de Ranculcus, lequel, pour des raisons semblables, a lui-même été rapproché de Lestodon (Hoffstetter 1958; Hirschfeld 1985). Or, à l'encontre du type de Ranculcus scalabrinianus Ameghino, 1891, qui consiste en un fragment de la branche horizontale d'une hémimandibule droite où manque la région symphysaire, Lestodon possède une symphyse mandibulaire fortement élargie en pelle carrée en avant des C. Les différents états du caractère correspondant à l'élargissement de la symphyse chez les Lestodontini peuvent être ainsi énumérés: chez Sphenotherus, le bord supérieur de la symphyse en vue occlusale dessine un contour continûment arrondi des $\mathrm{C}_{-}$à son extrémité antérieure, avec une légère constriction en avant de ces dernières. Chez Thinobadistes, la symphyse, plus large que la série dentaire de Pseudoprepotherium et des Scelidotheriinae, prend une forme grossièrement carrée, non rétrécie en avant des C. Chez Lestodon, la pelle symphysaire carrée ressemble à celle de $T h i$ nobadistes mais elle est plus large et la position très

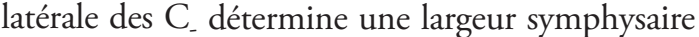
plus importante encore que chez Glossotherium. Chez les Mylodontinae Glossotheridium, Simomylodon 
n. gen., Kiyumylodon, Glossotherium et Mylodon, la pelle symphysaire montre toujours un rétrécissement en avant des C. En effet Glossotheridium possède un bord symphysaire antérieur très légèrement arrondi dont les bords supérieurs latéraux présentent une constriction en avant des C. Simomylodon n. gen. et Kiyumylodon seraient donc semblables à Glossotheridium, mais la pelle symphysaire y est proportionnellement moins longue. Chez Glossotherium, ce bord symphysaire, moins curviligne et plus large que chez Glossotheridium, est séparé des C_ par une zone de constriction proportionnellement plus courte que chez ce dernier. Enfin, chez Mylodon, la symphyse est relativement étroite.

Par la morphologie de sa symphyse (pelle symphysaire bien formée mais moins large que chez Glossotherium et présentant une zone de constriction en avant des $\left.\mathrm{C}_{-}\right)$, Simomylodon n. gen. est un Mylodontinae dont le degré évolutif est comparable à celui de Glossotheridium et probablement Kiyumylodon, inférieur à celui de Glossotherium et supérieur à celui observé chez Pleurolestodon.

Le rapprochement de Sphenotherus et de Ranculcus des Mylodontidae Lestodontini se justifie par des caractères morphologiques liés à la mandibule ou aux dents inférieures (position très latérale de la C. sur la mandibule chez les deux genres, ressemblances des dents inférieures de Ranculcus et de Thinobadistes). Les données chronostratigraphiques peuvent accessoirement être utilisées: Sphenotherus et Ranculcus sont du Huayquérien, Thinobadistes de l'Hemphillien. Toutes ces raisons nous incitent à penser que l'élargissement de la symphyse et du museau et probablement la forte divergence des séries dentaires se sont réalisés indépendamment chez les Mylodontidae Lestodontini et les formes apparentées, ainsi que chez Glossotherium. Les similitudes observées au niveau du crâne et des dents supérieures entre ces taxons pourraient être liées à une évolution parallèle des deux lignées alors que Webb (1989) les considèrent comme des synapomorphies.

\section{CARACTÈRES POSTCRÂNIENS}

L'examen préliminaire des éléments postcrâniens de Simomylodon $\mathrm{n}$. gen. indique qu'ils présentent de nombreuses similitudes avec ceux de Glossotherium et n'en diffèrent que par une plus faible taille.
Sur l'humérus, la perte du foramen entépicondylien est probablement une synapomorphie qui semble lier les Mylodontidae Lestodontini comme Thinobadistes et Lestodon aux Mylodontinae Simomylodon n. gen., Glossotherium et Mylodon.

Le fémur de Simomylodon n. gen. est plus grêle que celui de Glossotherium, mais son extrémité distale est caractéristique des Mylodontinae. De même, le niveau relativement bas de l'extrémité supérieure du grand trochanter par rapport à la tête du fémur correspond également à un état de caractère partagé par Simomylodon n. gen., Glossotherium, Paramylodon et Mylodon.

Le condyle interne odontoïde de l'astragale caractérise l'ensemble de la famille. On le trouve chez les Scelidotheriinae et les Mylodontinae (Kraglievich 1926; Webb 1989). Le condyle médial odontoïde de Simomylodon n. gen. est proportionnellement aussi élevé que celui de Pleurolestodon et de Glossotherium, beaucoup plus que chez Pseudoprepotherium (qui semble présenter l'état plésiomorphe de ce caractère pour la famille) et nettement plus que celui de Thinobadistes, qui présente un état moins dérivé que celui des Mylodontinae. Cette observation supplémentaire suggère une possible évolution parallèle de certains caractères entre les Lestodontini et la lignée menant à Glossotherium.

Sur l'astragale, la séparation des facettes ectale et sustentaculaire pour le calcanéum apparaît comme un état plésiomorphe rencontré également chez les Scelidotheriinae, Pseudoprepotherium, le groupe Thinobadistes-Lestodon, Pleurolestodon et Simomylodon n. gen. Il est donc difficile de proposer le groupe Pleurolestodon-Mylodon comme étant le groupe frère de Glossotherium sur la seule base de la fusion des facettes de l'astragale comme le fait Webb (1989), à moins de supposer une réversion par réapparition du sulcus tali chez Pleurolestodon, ce qui paraît peu probable, ou une fusion des facettes de l'astragale réalisée à deux reprises au moins (chez Glossotherium et Mylodon). Si l'on suppose que ce caractère n'est apparu qu'une fois, l'existence des deux facettes chez Simomylodon n. gen. devient un caractère généralisé interdisant d'inclure ce taxon dans le groupe Glossotherium-Mylodon. L'attribution au taxon Glossotherium chapadmalense par Robertson (1976) du Mylodontidae UF 10922 (Haile XV A) dont le matériel 
compte un astragale, suggère que Glossotheridium chapadmalense présente la condition dérivée de ce caractère et qu'il fait partie de ce groupe.

En conséquence, Simomylodon n. gen. est un Mylodontinae dont les caractères crâniens (degré d'élargissement du museau), mandibulaires (formation d'une pelle symphysaire moins large que chez Glossotherium et dont les bords montrent une constriction en avant des $\mathrm{C}_{-}$), dentaires (degré de divergence des séries dentaires supérieures, forte concavité du bord antérieur de la m3, développement uniquement lingual du lobe postérieur de cette dent, disparition du sillon antérieur sur la $C_{-}$) et postcrâniens (largeur du fémur, degré de développement du condyle odontoïde de l'astragale) indiquent qu'il pourrait s'agir d'un taxon plus spécialisé que Pleurolestodon (et peut-être que Glossotheridium). Il est en revanche moins dérivé que Glossotherium qui a acquis un diastème entre C- et M1, une importante divergence des séries dentaires et un fort élargissement du museau indépendamment des Mylodontidae Lestodontini. L'élargissement du museau et la divergence des séries dentaires chez Simomylodon n. gen. et Pleurolestodon sont comparables, ce qui signifierait pour ces deux taxons un stade évolutif similaire au sein des Mylodontinae. Chez Simomylodon n. gen., la spécialisation des prémaxillaires sans variation dans la morphologie des dents, indique vraisemblablement chez ce taxon un régime alimentaire différent de celui des autres Mylodontinae corrélé avec les changements climatiques (qui conditionnent la végétation) et biocénotiques affectant l'Altiplano entre le Miocène supérieur et le Pliocène. À cette époque, la faune était forcément adaptée à des conditions climatiques plus sèches que celles qui prévalaient durant le Huayquérien. Simomylodon n. gen., qui apparaît dès la base du Montehermoséen, à Pomata, à environ $75 \mathrm{~m}$ audessus de la Toba 76, se substitue à Pleurolestodon, dont le spécimen type a été découvert une quinzaine de mètres sous la Toba 76 . Il est possible que la disparition des faunes huayquériennes et donc de celle à Pleurolestodon soit liée, non seulement à l'établissement de conditions climatiques et hydriques plus rigoureuses que durant le Huayquérien, mais aussi, plus directement, au caractère catastrophique du dépôt de la Toba 76.

\section{VARIATIONS INDIVIDUELLES}

CHEZ SIMOMYLODON N. GEN. Lors de la description de Simomylodon uccasamamensis n. gen., n. sp., nous avons signalé les différences séparant l'holotype GB 078 de Viscachani du paratype MNHN-BOL V 3321 d'Ayo Ayo. Ce sont principalement une $\mathrm{C}$ - arquée chez le paratype, droite chez l'holotype; une M1 séparée de la M2 par un diastème plus long chez le paratype que chez l'holotype, où il est réduit à l'épaisseur d'une paroi inter-alvéolaire semblable aux autres; la face antérieure de la M3 orientée antérolatéralement postéromédialement chez le paratype, antéromédialement postérolatéralement chez l'holotype.

Une autre différence apparaît dans la forme de la section de la $\mathrm{m} 2$ entre un individu provenant d'Ayo Ayo MNHN AYO 165 et d'autres spécimens de cette localité, de Viscachani et de Pomata. De plus, les deux Mc III provenant d'Ayo Ayo sont soudés aux magnums, alors que ceux de Viscachani sont libres. Ces différences qui portent sur un matériel trop peu abondant pour que puissent être effectuées des études statistiques, ne doivent pas oblitérer les ressemblances existant entre GB 078 et MNHNBOL V 3321 au niveau des dents et des os du palais (en particulier les prémaxillaires dessinent un bord incisif droit), des métapodes (la partie métapodiale des Mc III-magnums provenant d'Ayo Ayo est semblable aux Mc III provenant de Viscachani) et de l'astragale (identiques à Viscachani et Ayo Ayo). Or, la morphologie de l'astragale dans la systématique des Tardigrades est très importante pour l'établissement de leur phylogénie (Pujos et al. 2007).

Dans l'attente de la découverte de nouveaux spécimens qui pourraient soutenir (ou non) cette hypothèse, nous considérons que les restes de Mylodontinae de Viscachani et d'Ayo Ayo appartiennent à un même taxon, Simomylodon uccasamamensis n. gen., n. sp.

La région symphysaire des mandibules du Mylodontinae de Pomata présente des similitudes suffisantes de taille et de morphologie avec les pièces homologues provenant d'Ayo Ayo et Viscachani pour envisager que cet animal appartient à la même espèce. La différence dans la morphologie des prémaxillaires observée entre Pleurolestodon et Simomylodon $\mathrm{n}$. gen. permet d'exclure toute appartenance 
de l'hémimandibule de Pomata (MNHN-BOL V 3321) à Pleurolestodon dalenzae n. sp., comme la proximité des dépôts à mammifères pourrait le laisser supposer. En effet, Pleurolestodon dalenzae n. sp. et le Mylodontinae de Pomata ont été découverts respectivement à $15 \mathrm{~m}$ sous la Toba 76 et $75 \mathrm{~m}$ au-dessus.

\section{CONCLUSIONS}

Lapparition dans le Mio-Pliocène de l'Altiplano bolivien de Pleurolestodon dalenzae n. sp. et de Simomylodon uccasamamensis n. gen., n. sp., est un élément important pour l'histoire des Mylodontinae en Amérique, et surtout pour la connaissance des Tardigrades néogènes provenant du nord de l'aire géographique patagonienne. Ce clade est donc présent dans cette région à une époque où la surrection des Andes centrales atteint déjà une altitude supérieure à $2000 \mathrm{~m}$. Les sédiments fossiliferes d'Ayo Ayo-Viscachani dans lesquels ont été retrouvé Simomylodon n. gen. (MacFadden et al. 1994) correspondent à un dépôt andin estimé à une élévation de 2850 m (Saint-André 1994). Ceci indique que les Mylodontinae se sont adaptés à des écosystèmes d'altitude dès la fin du Huayquérien.

À ce jour, il est impossible de préciser l'âge exact de Pleurolestodon dalenzae n. sp.: Huayquérien ou Montehermoséen? Son absence dans le registre taphonomique huayquérien relativement bien connu dans la région d'Achiri est notable. Est-ce la preuve de lacunes dans notre connaissance du terrain, ou bien l'indication d'une évolution originale des faunes altiplaniques au cours du Huayquérien? Cette dernière hypothèse est plausible dans la mesure où les Andes centrales subissent les effets de la seconde crise tectonique bolivienne entre 10-11 et 5-6 millions d'années (Marshall \& Sempéré 1991), c'està-dire principalement durant le Chasicoïen et le Huayquérien. Cette phase tectonique, marquée aussi par une reprise de l'activité volcanique, a presque doublé l'altitude de l'Altiplano. Un tel événement a eu certainement des conséquences écologiques importantes bien que difficiles à appréhender. Pleurolestodon dalenzae n. sp. est peut-être apparu sur l'Altiplano à la faveur des changements fauniques majeurs consécutifs à une crise environnementale qui affecte la région au cours du Huayquérien.

L'apparition de Simomylodon n. gen. et le renouvellement complet des genres de mammiferes entre le Huayquérien et le Montehermoséen illustrent bien la crise écologique qui se manifeste à la limite de ces deux étages. Ce changement faunistique, mis en lumière dans le dépôt de la Toba 76 et la crise tectonique précédemment évoquée, se traduit par des extinctions massives et par le remplacement de l'écosystème huayquérien caractérisé par la présence des Notoungulata-Mesotheriidae Plesiotypotherium achirense et P. majus (Villarroel 1974, 1978; Marshall et al. 1983) par un nouvel écosystème défini notamment, et dans tous les gisements, par Simomylodon $\mathrm{n}$. gen.

\section{Remerciements}

P.-A. Saint-André remercie Philippe Taquet et Robert Hoffstetter qui lui ont permis d'étudier les collections paléontologiques "Amérique du Sud» du MNHN, Christian de Muizon (MNHN) pour son appui tant moral que financier, Alejandra Dalenz Farjat et Federico Anaya pour leur aide lors des missions de terrain, Lionel Merlette et Françoise Pilard à qui revient le mérite d'avoir réalisé respectivement les photographies et les dessins qui illustrent cet article, ainsi qu'à Hervé Lelièvre qui a tenu à ce que ces recherches soient publiées dans la revue dont il avait la charge.

Les co-auteurs remercient l'ensemble du comité éditorial de la revue Geodiversitas, pour leurs encouragements à terminer ce travail dont le fondement scientifique revient essentiellement à P.-A. SaintAndré.

\section{RÉFÉRENCES}

AMEGHINO F. 1889. - Contribución al conocimiento de los mamiferos fósiles de la República Argentina. Coni P. e hijos, Buenos Aires, 1027 p.

Ameghino F. 1891. - Cincuenta especies nuevas de mamíferos fósiles argentinos. Revista Argentina de Historia Natural 1: 129-208.

Anaya F. \& MacFadden B. J. 1995. - Pliocene mammals from Inchasi, Bolivia. The endemic fauna just before the Great American Interchange. Bulletin of the 
Florida Museum of Natural History 39 (3): 87-140.

BARGO S., TOLEDO N. \& VizCAÍNO S. F. 2006. - Muzzle of South American Pleistocene Ground Sloths (Xenarthra, Tardigrada). Journal of Morphology 267: 248-263.

Cabrera A. 1936. - Las especies del género "Glossotherium". Notas del Museo de La Plata 1 (5): 193206.

Carlini A. A., Scillato-Yané G. J. \& Sánchez R. 2006. - New Mylodontoidea (Xenarthra, Phyllophaga) from the middle Miocene-Pliocene of Venezuela. Journal of Systematic Palaeontology 4 (3): 255-267.

CARTELle C. 1991. - Um novo Mylodontinae (Edentata, Xenarthra) do Pleistoceno final de região intertropical brasileira. Anais da Academia brasileira de Ciências 63 (2): 161-170.

Cione A. L., Azpelicueta M., Bond M., Carlini A. A., Casciotta J. R., Cozzuol M. A., Fuente M. DE la, Gasparini Z., Goin F. J., Noriega J., ScillatoYané G. J., Soibelzon L., Tonni E. P., Verzi D. \& VuCETICH M. G. 2000. - Miocene vertebrates from Entre Ríos Province, Eastern Argentina. INSUGEO, Serie Correlación Geológica 14: 191-237.

ENGELMANN G. F. 1985. - The phylogeny of the Xenarthra, in MONTGOMERY G. G. (ed.), The Evolution and Ecology of Armadillos, Sloths, and Vermilinguas. Smithsonian Institution Press, Washington, DC: 51-64.

Engelmann G. F. 1987. - A new Deseadan Sloth (Mammalia: Xenarthra) from Salla, Bolivia, and its implications for the primitive condition of the dentition in Edentates. Journal of Vertebrate Paleontology 7 (2): 217-223.

Esteban G. I. 1996. - Revisión de los Mylodontinae cuaternarios (Edentata-Tardigrada) de Argentina, Bolivia y Uruguay. Sistemática, filogenia, paleobiología, paleozoogeografía y paleoecología. Doctorado en Ciencias Naturales "Orientación Zoología", Universidad Nacional de Tucumán, Facultad de Ciencias Naturales e Instituto Miguel Lillo, Tucumán, Argentine, 314 p.

Evernden J. F., Kriz S. J. \& Cherroni C. 1977. Potassium-Argon ages of some Bolivian rocks. Economic Geology 72: 1042-1061.

Frailey C. D. 1986. — Late Miocene and Holocene Mammals, exclusive of the Notoungulata, of the Río Acre Region, Western Amazonia. Contribution in Science (374): 1-46.

GAUDIN T. J. 2004. - Phylogenetic relationships among sloths (Mammalia, Xenarthra, Tardigrada): the craniodental evidence. Zoological Journal of the Linnean Society 140: 255-305.

GeRVAis P. \& AMEGHINO F. 1880. - Les mammiferes fossiles d'Amérique du Sud. F. Savy, Paris, 225 p.

GUTH C. 1962. - Apport systématique et phylogénétique de l'étude de la région temporale chez les Édentés. Colloques internationaux du CNRS (104): 341-361.

HiRSCHFELD S. E. 1985. - Ground sloths from the Friasian La Venta Fauna, with additions to the Pre-
Friasian Coyaima Fauna of Colombia, South America. University of California Publications in Geological Sciences 128: 1-91.

HOFFSTETTER R. 1952. — Les Mammifères pléistocènes de la république de l'Équateur. Mémoires de la Société géologique de France, nouvelle série (66): 1-391.

Hoffstetter R. 1956. - Contribution à l'étude des Orophodontoidea, Gravigrades cuirassés de la Patagonie. Annales de Paléontologie 42: 27-64.

Hoffstetter R. 1958. - Xenarthra, in Piveteau J. (ed.), Traité de Paléontologie 6 (2). Masson et Cie, Paris: 535-636.

Hoffstetter R. 1982. — Les Edentés Xénarthres, un groupe singulier de la faune néotropicale (origine, affinités, radiation adaptative, migrations et extinctions). Proceedings of the First International Meeting on "Palaeontology, Essential of Historical Geology", Venise, 1981: 385-443.

Hoffstetter R. 1986. - High Andean Mammalian Faunas during the Plio-Pleistocene, in VuILLEUMIER F. \& Monasterio M. (eds), High Altitude Tropical Biogeography. Vol. 9, New York, Oxford University Press: 219-245.

Hoffstetter R., Martinez C., Muñoz-Reyes J. \& Tomasi P. 1971. — Le gisement d'Ayo Ayo (Bolivie), une succession stratigraphique Pliocène-Pléistocène datée par des Mammifères. Comptes Rendus de l'Académie des Sciences 273, série D: 2472-2475.

Hoffstetter R., Martinez C. \& Tomasi P. 1972. Nouveaux gisements de mammifères néogènes dans les couches rouges de l'Altiplano bolivien. Comptes Rendus de l'Académie des Sciences 275, série D: 739-742.

Kraglievich L. 1921. — Estudios sobre los Mylodontinae. Descripción comparativa del género "Pleurolestodon" Rov. Anales del Museo Nacional de Historia Natural de Buenos Aires 31: 95-118.

KRAGLIEVICH L. 1923. - Descripción comparada de los cráneos de Scelidodon rothi Amegh. y Scelidotherium parodii, n. sp. Anales del Museo Nacional de Historia Natural de Buenos Aires 33: 57-103.

KRAGLievich L. 1925. - Cuatro nuevos gravigrados de la fauna araucana "chapadmalense". Anales del Museo Nacional de Historia Natural de Buenos Aires 33: 215-235.

KRAGLievich L. 1926. — Notas sobre los Gravígrados de Sud América. Anales del Museo Nacional de Historia Natural de Buenos Aires 34: 21-36.

KRAGLieVich L. 1934. - Contribución al conocimiento de "Mylodon darwini" Owen y especies afines. Revista del Museo de La Plata 34: 255-292.

LAVENU A. 1984. - Âge pliocène de la formation Remedios dans l'Altiplano bolivien. Caractères de la tectonique pliocène. Comptes Rendus de l'Académie des Sciences 293, série II (15): 1051-1054.

LAVENU A. 1986. - Étude néotectonique de l'Altiplano et de la cordillère orientale des Andes boliviennes. Thèse 
d'État inédite, Université Paris-Sud, Orsay, 434 p.

LYDEKKER R. 1894. - The extinct Edentates of Argentina. Anales del Museo de La Plata 3 (2): 1-118.

MacFadden B. J., Wang Y., Cerling T. E. \& Anaya F. 1994. - South American fossil mammals and carbon isotopes: a 25 million-year sequence from the Bolivian Andes. Palaeogeography, Palaeoclimatology and Palaeoecology 107: 257-268.

Marshall L. G., Hoffstetter R. \& Pascual R. 1983. - Mammals and stratigraphy: geochronology of the continental mammal-bearing Tertiary of South America. Palaeovertebrata Mémoire extraordinaire: 1-93.

Marshall L. G. \& Sempéré T. 1991. - The Eocene to Pleistocene Vertebrates of Bolivia and their stratigraphic context: a review, in SUAREZ R. (ed.), Fósiles y Facies de Bolivia - Vol. 1: Vertebrados. Revista técnica de YPFB 12 (3-4): 631-652.

Marshall L. G., Swisher III C. C., Lavenu A., HoffSTETTER R. \& CURTis G. H. 1992. — Geochronology of the mammal-bearing late Cenozoic on the Northern Altiplano, Bolivia. Journal of South American Earth Sciences 5 (1): 1-19.

McDonald H. G. \& Perea D. 2002. - The large scelidothere Catonyx tarijensis (Xenarthra, Mylodontidae) from the Pleistocene of Uruguay. Journal of Vertebrate Paleontology 22 (3): 677-683.

McKenna M. C. \& Bell S. K. 1997. - Classification of Mammals Above the Species Level. Columbia University Press, New York, $631 \mathrm{p}$.

McKenna M. C., Wyss A. R. \& Flynn J. J. 2006. Paleogene Pseudoglyptodont Xenarthrans from Central Chile and Central Patagonia. American Museum Novitates (3536): 1-18.

MONES A. 1986. - Acremylodon a new name for Stenodon Frailey, 1986 (Mammalia: Edentata: Mylodontidae). Comunicaciones Paleontológicas del Museo de Historia Natural de Montevideo 16 (1): 227-228.

Negri F. R. \& FerigOlo J. 2004. — Urumacotheriinae, nova subfamília de Mylodontidae (Mammalia, Tardigrada) do Mioceno superior-Plioceno, América do Sul. Revista Brasileira de Paleontología 7 (2): 281-288.

Parodi Bustos R. 1974. — Notas sobre milodontinos sudamericanos. Reivindicación del subgénero Pseudolestodon F. Ameghino y H. Gervais. Ameghiniana 11 (1): 88-93.

PASCUAL R. 1966. - Vertebrata, in Borello A. V. (ed.), Paleontografía Bonaerense. Comisión de Investigación Científica de la Provincia de Buenos Aires, Buenos Aires: 171-178.

Paula Couto C. De 1979. - Tratado de Paleomastozoologia. Academia Brasileira de Ciências, Rio de Janeiro, $590 \mathrm{p}$.

Pujos F. 2000. - Scelidodon chiliensis (Xenarthra, Mammalia) du Pléistocène terminal de «Pampa de los Fósiles». Quaternaire 11 (3-4): 197-206.
Pujos F. 2007. - On the revision of Octodontotherium: the oldest mylodontoid sloth. 8th International Congress of Vertebrate Morphology, Paris, France, 16-21 Juillet 2007. Journal of Morphology 268 (12): 1121.

Pujos F. \& De IULIIS G. 2007. — Late oligocene megatherioidea fauna (Edentata: Xenarthra) from SallaLuribay (Bolivia): new data on basal sloth radiation and Cingulata-Phyllophaga split. Journal of Vertebrate Paleontology 27 (1): 132-144.

Pujos F., De Iulis G., Argot C. \& Werdelin L. 2007. A peculiar climbing Megalonychidae from the Pleistocene of Peru and its implications for sloth history. Zoological Journal of the Linnean Society 149: 179-235.

Pujos F., Carlini A. A., Cerdeño E. \& Prámparo M. 2008. - Preliminary report of Deseadan sloths from Quebrada Fiera (Mendoza Province, Argentina). Tercer Congreso Latinoamericano de Paleontología de Vertebrados, Neuquén, Patagonia Argentina, 22-25, Septembre 2008, Libro de Resúmenes: 206.

REINHARDT J. 1879. - Beskrivelse af hovedskallen af et kaempedovendyr, Grypotherium darwinii, fra La Plata-Landenese Plejstocene Dannelser. Videnskabelige Selsk. Skr. Raekke Naturvidenskabelig og Mathematisk Afhandl. 12 (4): 351-380.

Rinderknecht A., Perea D. \& McDonald H. G. 2007. - A new Mylodontinae (Mammalia, Xenarthra) from the Camacho Formation (Late miocene), Uruguay. Journal of Vertebrate Paleontology 27(3): 744-747.

ROBERTSON J. S. 1976. — Latest Pliocene mammals from Haile XV A, Alachua County, Florida. Bulletin of the Florida State Museum 20 (3): 111-186.

RovereTO C. 1914. - Los estratos Araucanos y sus fósiles. Anales del Museo Nacional de Historia Natural de Buenos Aires 25: 1-250.

SAINT-ANDRÉ P.-A. 1994 - Contribution à l'étude des grands mammiferes du Néogène de l'Altiplano bolivien. Thèse inédite de Doctorat, Muséum national d'Histoire naturelle, Paris, France, 564 p.

Scillato-Yané G. J. 1977. — Octomylodontinae: nueva subfamilia de Mylodontidae (Edentata, Tardígrada). Descripción del cráneo y mandíbula de Octomylodon robertoscagliai n. sp., procedentes de la Formación Arroyo Chasicó (Edad Chasiquense, Plioceno temprano) del sur de la Provincia de Buenos Aires (Argentina). Algunas consideraciones filogenéticas y sistemáticas sobre los Mylodontoidea. Publicaciones del Museo Municipal de Ciencias Naturales de Mar del Plata "L. Scaglia" 2 (5): 123-140.

ScotT W. B. 1904. - Mammalia of the Santa Cruz Beds, Part I: Edentata: 3 Gravigrada. Reports of the Princeton Expeditions to Patagonia 5: 227-364.

SiMPSON G. G. 1941. - A Miocene sloth from southern Chile. American Museum Novitates 1156: 1-6.

STOCK C. 1925. - Cenozoic Gravigrade Edentates of Western North America with special reference to the Pleistocene Megalonychinae, and Mylodontidae 
of Rancho La Brea. Carnegie Institute of Washington 331: 1-206

Villarroel C. 1974. — Les Mésothériinés (Notoungulata, Mammalia) du Pliocène de Bolivie. Leurs rapports avec ceux d'Argentine. Annales de Paléontologie des Vertébrés 60 (2): 245-286.

VillarRoel C. 1978. — Edades y correlaciones de algunas unidades litoestratigráficas del Altiplano boliviano y estudios de algunos representantes Mesotheriinos. Revista de la Academia Nacional de Ciencias de Bolivia 1 (1): 159-170.

Weвв S. D. 1989. - Osteology and relationships of Thinobadistes segnis, the first mylodont sloth in North America, in REDFORD K. H. \& EISENBERG J. F. (eds), Advances in Neotropical Mammalogy. Sandhill Crane Press, Gainesville: 469-532.

Soumis le ler octobre 2008; accepté le 4 février 2009. 